\title{
ACTION FUNCTIONAL AND QUASI-POTENTIAL FOR THE BURGERS EQUATION IN A BOUNDED INTERVAL
}

\author{
LORENZO BERTINI, ALBERTO DE SOLE, DAVIDE GABRIELLI, \\ GIOVANNI JONA-LASINIO, AND CLAUDIO LANDIM
}

\begin{abstract}
Consider the viscous Burgers equation $u_{t}+f(u)_{x}=\varepsilon u_{x x}$ on the interval $[0,1]$ with the inhomogeneous Dirichlet boundary conditions $u(t, 0)=$ $\rho_{0}, u(t, 1)=\rho_{1}$. The flux $f$ is the function $f(u)=u(1-u), \varepsilon>0$ is the viscosity, and the boundary data satisfy $0<\rho_{0}<\rho_{1}<1$. We examine the quasi-potential corresponding to an action functional, arising from nonequilibrium statistical mechanical models, associated to the above equation. We provide a static variational formula for the quasi-potential and characterize the optimal paths for the dynamical problem. In contrast with previous cases, for small enough viscosity, the variational problem defining the quasi potential admits more than one minimizer. This phenomenon is interpreted as a non-equilibrium phase transition and corresponds to points where the super-differential of the quasi-potential is not a singleton.
\end{abstract}

\section{INTRODUCTION}

We consider an infinite dimension version of the classical Freidlin-Wentzell variational problem for the quasi-potential. We first briefly recall this topic in the context of diffusion processes in $\mathbb{R}^{n} 23$. Let $b$ be a smooth vector field in $\mathbb{R}^{n}$ and consider the stochastic perturbation of the dynamical systems $\dot{x}=b(x)$ given by

$$
\dot{X}_{\gamma}=b\left(X_{\gamma}\right)+\sqrt{2 \gamma} \dot{w},
$$

where $\dot{w}$ is a white noise. Under suitable assumptions on $b$, the process $X_{\gamma}$ has a unique invariant measure $\mu_{\gamma}$. The Freidlin-Wentzell theory provides a variational expression for the asymptotics of this invariant measure in the weak noise limit $\gamma \downarrow 0$. To each path $X:(-\infty, 0] \rightarrow \mathbb{R}^{n}$ associate the action

$$
I(X)=\frac{1}{4} \int_{-\infty}^{0}|\dot{X}(t)-b(X(t))|^{2} d t .
$$

In the sequel we assume that the vector field $b$ has a unique, globally attractive, equilibrium point $\bar{x}$. The quasi-potential $V: \mathbb{R}^{n} \rightarrow[0,+\infty)$ is defined by

$$
V(x)=\inf \{I(X), X(0)=x, X(t) \rightarrow \bar{x} \text { as } t \rightarrow-\infty\} .
$$

Namely, $V(x)$ is the minimal action to reach $x$ starting from the equilibrium point $\bar{x}$. For each Borel set $B \subset \mathbb{R}^{n}$ we then have, as $\gamma \downarrow 0$,

$$
\mu_{\gamma}(B) \asymp \exp \left\{-\gamma^{-1} \inf _{x \in B} V(x)\right\} .
$$

If the vector field $b$ is conservative, namely $b=-\nabla U$ for some $U: \mathbb{R}^{n} \rightarrow \mathbb{R}$, then $V(x)=U(x)-U(\bar{x})$, i.e. the quasi-potential coincides with the potential. In general, though, there is no simple expression for the quasi-potential. 
In this finite dimensional setting, the quasi-potential $V$ is Lipschitz 23], in particular it is a.e. differentiable. As discussed in [14, for some "special" points $x \in \mathbb{R}^{n}$, the function $V$ might however have "corners", namely the super-differential of $V$ might not be a singleton. From a dynamical point of view, for such points $x$ there would exist more than a single minimizer for the variational problem (1.2). We refer to the examples considered in the physical literature [24, 25, 31] for a discussion on the physical interpretation of this lack of uniqueness. In this article we show that this phenomenon occurs for an infinite dimensional dynamical system. As far as we know, this is the first concrete example in which such a result is analytically proven.

As shown in 13, 14, 32, if $\mathbb{H}$ denotes the Hamiltonian associated to the action (1.1), then the quasi-potential $V$ is a viscosity solution, the correct PDE formulation of the variational problem (1.2) in presence of "corners", to the Hamilton-Jacobi equation $\mathbb{H}(x, D V)=0, x \in \mathbb{R}^{n}$.

We examine in this article an infinite dimensional version of the previous variational problem. As the basic dynamical system, we consider the following non-linear parabolic equation on the interval $[0,1]$

$$
u_{t}+f(u)_{x}=\varepsilon\left(D(u) u_{x}\right)_{x}
$$

with the inhomogeneous Dirichlet boundary condition $u(t, 0)=\rho_{0}, u(t, 1)=\rho_{1}$. In the above equation, $f$ is the flux, $D>0$ the diffusion coefficient and $\varepsilon>$ 0 the viscosity. To introduce the associated action functional, add an external "controlling" field $E=E(t, x)$ to obtain the perturbed equation

$$
u_{t}+f(u)_{x}+2 \varepsilon(\sigma(u) E)_{x}=\varepsilon\left(D(u) u_{x}\right)_{x},
$$

where $\sigma(u) \geq 0$ is the mobility. Denote by $u^{E}$ the solution of this equation. The action of a path $u:(-\infty, 0] \times[0,1] \rightarrow \mathbb{R}$ is given by

$$
I^{\varepsilon}(u)=\inf \varepsilon \int_{-\infty}^{0} \int_{0}^{1} \sigma(u) E^{2} d x d t
$$

where the infimum is carried over all $E$ such that $u^{E}=u$. The quasi-potential is then introduced as in the finite dimensional setting, namely $V_{\varepsilon}$ is the functional on the set of functions $\rho:[0,1] \rightarrow \mathbb{R}$ defined by

$$
V_{\varepsilon}(\rho)=\inf \left\{I^{\varepsilon}(u): u(0)=\rho, u(t) \rightarrow \bar{\rho}_{\varepsilon} \text { as } t \rightarrow-\infty\right\},
$$

where $\bar{\rho}_{\varepsilon}$ is the unique stationary solution of (1.3).

Informally, as in the Freidlin-Wentzell theory, one can add a stochastic perturbation to (1.3),

$$
u_{t}^{\gamma}+f\left(u^{\gamma}\right)_{x}=\varepsilon\left(D\left(u^{\gamma}\right) u_{x}^{\gamma}\right)_{x}+\left(\sqrt{2 \varepsilon \gamma \sigma\left(u^{\gamma}\right)} \dot{w}_{\gamma}\right)_{x},
$$

where $\dot{w}_{\gamma}$ is a white noise in time that becomes also white in space as $\gamma \downarrow 0$. Then, informally, the finite dimensional theory carries over to the present setting. In particular, the quasi-potential $V_{\varepsilon}$ in (1.6) describes the asymptotics for the invariant measure of the process $u^{\gamma}$ as $\gamma \downarrow 0$. We refer to [30] for an analysis of the large deviation properties of the stochastic PDE (1.7), with periodic boundary conditions, in the joint limit $\gamma \downarrow 0$ and $\varepsilon \downarrow 0$.

Our main motivation for the analysis of the action functional (1.5) and the quasi-potential (1.6) comes, however, from non-equilibrium statistical mechanics. For a class of interacting particle systems, the so-called stochastic lattice gases, 
it has been shown that equation (1.3) describes the typical evolution of the empirical density in the diffusive scaling limit [26. Moreover, the functional $I^{\varepsilon}$, as in the Freidlin-Wentzell theory, gives the corresponding asymptotic probability of observing deviations from the typical behavior [10, 26, 27.

In the case of equilibrium models, the flux $f$ vanishes and the boundary conditions are equal. For such models, which are analogous to gradient vector fields in the finite dimensional situation, the quasi-potential (1.6) does not depend on the viscosity $\varepsilon$ and coincides with the thermodynamic free energy functional of the underlying microscopic model, whose invariant measure has the standard Gibbs form. If the flux $f$ does not vanishes but the boundary data are still equal, $\rho_{0}=\rho_{1}$, the quasi-potential does not depend on $f$ and it is equal to the one of the corresponding equilibrium model, see [4, 7] for the analogous result in the case of periodic boundary conditions. On the other hand, if the boundary data are not equal, in general there is no simple expression for the invariant measure of the microscopic dynamics, often called stationary non-equilibrium state. In order to analyze the behavior of such invariant measure in the thermodynamic limit, in [5] we introduced the dynamical/variational approach outlined above. In particular, the quasi-potential $V_{\varepsilon}$ plays an analogous role to the free energy for equilibrium systems. This gives actually a natural way to extend the notion of thermodynamic potential to non-equilibrium systems.

As it has been shown by concrete examples [5, 6, 8, 9, 16, 20, for non-equilibrium models the quasi-potential (1.6) presents peculiar features. While for equilibrium models $V_{\varepsilon}$ is always a convex local functional, i.e. of the form $V_{\varepsilon}(\rho)=\int_{0}^{1} v(\rho(x)) d x$ for some convex real function $v$, for non-equilibrium models $V_{\varepsilon}$ might be non-local and non-convex. In terms of the underlying microscopic model, the non-locality of the quasi-potential corresponds to the presence of long-range correlations which are believed to be a generic feature of stationary non-equilibrium states [18.

The main purpose of the present paper is to show, by a concrete example, that for non-equilibrium models the quasi-potential might have "corners", equivalently that the minimizer for the variational problem (1.6) is not unique. We shall analyze the model defined by a constant diffusion coefficient, $D=1$, while the flux and mobility are given by $f(u)=\sigma(u)=u(1-u), u \in[0,1]$. Then the parabolic equation (1.3) becomes the viscous Burgers equation and the action functional $I^{\varepsilon}$ in $(1.5)$ can be obtained as the large deviation rate functional of the so-called weakly asymmetric simple exclusion process [10, 27. As shown in [11, 21, the quasi-potential (1.6) is also the large deviation rate function for the invariant measure.

When $\rho_{0}>\rho_{1}$, so that both the boundary conditions and the flux $f$ "push" the density $u \in[0,1]$ to the right, the behavior of the invariant measure has been discussed in [20] by combinatorial techniques. In particular, in 20] a static variational characterization for the rate function of the invariant measure is derived in terms of a one-dimensional boundary value problem. More recently, the same model is analyzed in 8 , where it is shown that the quasi-potential (1.6) can be written in terms of the variational expression derived in 20] and the optimal paths for (1.6) are characterized. We emphasize that in this case, i.e. for $\rho_{0}>\rho_{1}$, the quasi-potential has no corners and the minimizer for (1.6) is unique.

In this paper we examine the same model but in the more interesting situation in which $\rho_{0}<\rho_{1}$, so that there is an effective competition between the flux and the boundary conditions. Our main results are summarized as follows. By analyzing 
the variational problem (1.6), we establish a static variational characterization of $V_{\varepsilon}$ analogous to the one in [8, 20]. We emphasize that in the case here discussed there is no uniqueness for the minimizer of (1.6) and this introduces few technical complications. We then discuss the variational convergence of the quasi-potential $V_{\varepsilon}$ in the inviscid limit $\varepsilon \downarrow 0$. In particular, we show that in this limit we recover the functional derived in [17] in the context of the boundary driven asymmetric exclusion process. By a perturbation argument with respect to the limiting case $\varepsilon=0$ we then show, provided the viscosity $\varepsilon$ is small enough, that there exist functions $\rho$ such that the minimizer for (1.6) is not unique. We also show that in such points $\rho$ the super-differential of $V_{\varepsilon}$ is not a singleton. In the context of equilibrium statistical mechanics, the existence of more than a single tangent functional to the quasi-potential, which in this case coincides with the free energy functional, is due to the occurrence of phase transitions. We therefore interpret the fact that the super-differential of $V_{\varepsilon}$ is not a singleton as a non-equilibrium phase transition. Finally, we discuss the connection of the quasi-potential $V_{\varepsilon}$ to the Hamilton-Jacobi equation $\mathbb{H}_{\varepsilon}(\rho, D V)=0$, where $\mathbb{H}_{\varepsilon}$ is the Hamiltonian associated to the action (1.5).

\section{Notation And Results}

Viscous Burgers equation. Consider the viscous Burgers equation on the interval $[0,1]$ with inhomogeneous Dirichlet boundary conditions at the endpoints namely,

$$
\left\{\begin{array}{l}
u_{t}+f(u)_{x}=\varepsilon u_{x x} \\
u(t, 0)=\rho_{0}, \quad u(t, 1)=\rho_{1},
\end{array}\right.
$$

where $u=u(t, x)$ is a scalar function and hereafter we denote partial derivatives with subscripts. The flux $f$ is the function $f(u)=u(1-u), \varepsilon>0$ is the viscosity, and the boundary data, fixed throughout the paper, satisfy $0<\rho_{0}<\rho_{1}<1$.

Simple computations show that the unique stationary solution $\bar{\rho}_{\varepsilon}$ of the viscous Burgers equation (2.1) can be described as follows. Let $J_{0}=J_{0}\left(\rho_{0}, \rho_{1}\right):=$ $\min _{r \in\left[\rho_{0}, \rho_{1}\right]} f(r)$. For each $\varepsilon>0$ there exists a unique $J_{\varepsilon} \in\left(-\infty, J_{0}\right)$ such that

$$
\int_{\rho_{0}}^{\rho_{1}} \frac{\varepsilon}{f(r)-J_{\varepsilon}} d r=1 .
$$

The function $\bar{\rho}_{\varepsilon}$ is then obtained by integrating $f\left(\bar{\rho}_{\varepsilon}\right)-\varepsilon\left(\bar{\rho}_{\varepsilon}\right)_{x}=J_{\varepsilon}$ with the boundary condition $\bar{\rho}_{\varepsilon}(0)=\rho_{0}$. In particular, the function $\bar{\rho}_{\varepsilon}$ is strictly increasing. We remark that the constant $J_{\varepsilon}$ can be interpreted as the current maintained by the stationary solution $\bar{\rho}_{\varepsilon}$. Let $\varphi_{i}:=\log \left[\rho_{i} /\left(1-\rho_{i}\right)\right] \in \mathbb{R}, i=0,1$, and set $\varepsilon_{0}:=1 /\left(\varphi_{1}-\varphi_{0}\right)$. Clearly, $J_{\varepsilon}$ is increasing as $\varepsilon$ decreases and $J_{\varepsilon_{0}}=0$; therefore $0<J_{\varepsilon}<J_{0}$ for $0<\varepsilon<\varepsilon_{0}$ and $J_{\varepsilon}<0$ for $\varepsilon>\varepsilon_{0}$. A simple computation shows also that $\lim _{\varepsilon \downarrow 0} J_{\varepsilon}=J_{0}$.

By standard arguments for parabolic equations, see e.g. the more sophisticated analysis in [15], the stationary solution $\bar{\rho}_{\varepsilon}$ is globally attractive for the flow defined by (2.1). More precisely, fix $\varepsilon>0$, let $u(t ; \rho), t \geq 0$, be the solution to (2.1) with initial condition $u(0, \cdot ; \rho)=\rho(\cdot)$, and assume that $\rho:[0,1] \rightarrow \mathbb{R}$ is a continuous function satisfying the boundary conditions $\rho(0)=\rho_{0}, \rho(1)=\rho_{1}$. Then $u(t ; \rho)$ converges in the $C^{1}$ topology to $\bar{\rho}_{\varepsilon}$ as $t \rightarrow \infty$. Furthermore, this convergence is uniform with respect to $\rho$ in a bounded set of $C([0,1])$ and holds with an exponential rate. 
The action functional. To define rigorously the action functional informally introduced in (1.5), we need to introduce some notation. Given $T>0$, the inner products in $L^{2}([0,1])$ and $L^{2}([-T, 0] \times[0,1])$ are denoted by $\langle\cdot, \cdot\rangle$ and $\langle\langle\cdot, \cdot\rangle\rangle$ respectively. We consider the space $L^{\infty}([0,1])$ equipped with the weak* topology and let $M$ be the set

$$
M=\left\{\rho \in L^{\infty}([0,1]): 0 \leq \rho \leq 1\right\}
$$

equipped with the relative topology. Then $M$ is a compact Polish space, i.e. complete metrizable and separable. Recall that, by definition of the weak* topology, a sequence $\left\{\rho^{n}\right\} \subset M$ converges to $\rho$ in $M$ if and only if $\left\langle\rho^{n}, g\right\rangle \rightarrow\langle\rho, g\rangle$ for any $g \in L^{1}([0,1])$. For $T>0$, we let $C([-T, 0] ; M)$ be the set of continuous paths $u:[-T, 0] \rightarrow M$ equipped with the topology of uniform convergence.

Let $C_{0}^{\infty}([-T, 0] \times[0,1])$ be the space of smooth functions $H:[-T, 0] \times[0,1] \rightarrow \mathbb{R}$ satisfying $H(t, 0)=H(t, 1)=0$ for $t \in[-T, 0]$ and $H(-T, x)=H(0, x)=0$ for $x \in[0,1]$. Given $u \in C([-T, 0] ; M)$, let $L_{u}^{\varepsilon}: C_{0}^{\infty}([-T, 0] \times[0,1]) \rightarrow \mathbb{R}$ be the linear functional defined by

$$
\begin{aligned}
L_{u}^{\varepsilon}(H) & :=-\left\langle\left\langle u, H_{t}\right\rangle\right\rangle-\left\langle\left\langle f(u), H_{x}\right\rangle\right\rangle-\varepsilon\left\langle\left\langle u, H_{x x}\right\rangle\right\rangle \\
& +\varepsilon \int_{-T}^{0}\left[\rho_{1} H_{x}(t, 1)-\rho_{0} H_{x}(t, 0)\right] d t .
\end{aligned}
$$

If $u$ is a smooth function satisfying the boundary conditions $u(t, 0)=\rho_{0}$ and $u(t, 1)=\rho_{1}$ for $t \in[-T, 0]$, then $L_{u}^{\varepsilon}(H)=\left\langle\left\langle u_{t}+f(u)_{x}-\varepsilon u_{x x}, H\right\rangle\right.$. Moreover, the functional $L_{u}^{\varepsilon}$ vanishes if and only if $u$ is a weak solution to (2.1).

Let $\sigma:[0,1] \rightarrow[0,+\infty)$ be the mobility of the system; we assume it is the function defined by $\sigma(a)=a(1-a)$. Given $u \in C([-T, 0], M)$, let

$$
\left\langle\left\langle u_{x}, u_{x}\right\rangle\right\rangle:=\sup _{H}\left\{-2\left\langle\left\langle u, H_{x}\right\rangle\right\rangle-\langle\langle H, H\rangle\},\right.
$$

where the supremum is carried over all smooth functions $H:[-T, 0] \times[0,1] \rightarrow$ $\mathbb{R}$ such that $H(t, 0)=H(t, 1)=0, t \in[-T, 0]$. The action functional $I_{[-T, 0]}^{\varepsilon}$ : $C([-T, 0] ; M) \rightarrow[0,+\infty]$ is then defined by

$$
I_{[-T, 0]}^{\varepsilon}(u):= \begin{cases}\sup _{H}\left\{L_{u}^{\varepsilon}(H)-\varepsilon\left\langle\left\langle H_{x}, \sigma(u) H_{x}\right\rangle\right\rangle\right\} & \text { if }\left\langle\left\langle u_{x}, u_{x}\right\rangle\right\rangle<+\infty \\ +\infty & \text { otherwise }\end{cases}
$$

where the supremum is carried over all functions $H$ in $C_{0}^{\infty}([-T, 0] \times[0,1])$. Clearly, $I_{[-T, 0]}^{\varepsilon}(u)$ vanishes if and only if $u \in C([-T, 0] ; M)$ admits a square integrable derivative and is a weak solution to (2.1). We refer to [10] for equivalent definitions of the action functional $I_{[-T, 0]}^{\varepsilon}$. We remark that in [10 the action functional is defined with the condition $\left\langle\left\langle u_{x}, u_{x}\right\rangle\right\rangle\langle+\infty$ replaced by the stronger condition $\left\langle\left\langle u_{x}, \sigma(u)^{-1} u_{x}\right\rangle\right\rangle<+\infty$. The argument in [10, Lemma 4.9] shows however that if the supremum on the right hand side of (2.3) is finite these conditions are in fact equivalent.

In order to state the connection of the action functional to the perturbed parabolic problem (1.4) we need few more definitions. Denote by $C^{1}([0,1])$ the space of continuously differentiable functions $h:[0,1] \rightarrow \mathbb{R}$ and let $C_{0}^{1}([0,1]):=\{h \in$ $\left.C^{1}([0,1]): h(0)=h(1)=0\right\}$. Given a positive bounded measurable function $\gamma:[0,1] \rightarrow[0,+\infty)$, let $\mathcal{H}_{0}^{1}(\gamma)$ be the Sobolev space induced by $C_{0}^{1}([0,1])$ endowed 
with the inner product

$$
\langle h, g\rangle_{1, \gamma}=\int_{0}^{1} h_{x} g_{x} \gamma d x .
$$

To be precise, the induced space $\mathcal{H}_{0}^{1}(\gamma)$ is obtained by identifying and completing elements $h \in C_{0}^{1}([0,1])$ with respect to the seminorm $\langle h, h\rangle_{1, \gamma}^{1 / 2}$. When $\gamma=1$ the space $\mathcal{H}_{0}^{1}(\gamma)$ is the standard Sobolev space on $[0,1]$, in this case we denote it simply by $\mathcal{H}_{0}^{1}$. Note that in the above equation, as well as below, we drop from the notation the explicit dependence on the integration variable when there is no ambiguity.

Denote by $|\cdot|_{1, \gamma}$ the norm of $\mathcal{H}_{0}^{1}(\gamma)$ and let $\mathcal{H}_{0}^{-1}(\gamma)$ be the dual space of $\mathcal{H}_{0}^{1}(\gamma)$. It is equipped with the dual norm $|\cdot|_{-1, \gamma}$ defined by

$$
|\ell|_{-1, \gamma}^{2}=\sup \left\{2\langle\ell, h\rangle-|h|_{1, \gamma}^{2}, h \in \mathcal{H}_{0}^{1}(\gamma)\right\},
$$

where $\langle\ell, h\rangle$ stands also for the value at $h$ of the linear functional $\ell$.

Fix a path $u$ in $C([-T, 0], M)$ and denote by $\mathfrak{H}_{0}^{1}(\sigma(u))$ the Hilbert space induced by $C_{0}^{\infty}([-T, 0] \times[0,1])$ endowed with the inner product $\left\langle\langle\cdot, \cdot\rangle_{1, \sigma(u)}\right.$ defined by

$$
\langle\langle H, G\rangle\rangle_{1, \sigma(u)}=\int_{-T}^{0}\langle H, G\rangle_{1, \sigma(u(t))} d t
$$

and let $\|\cdot\|_{1, \sigma(u)}$ be the associated norm. Let $\mathfrak{H}_{0}^{-1}(\sigma(u))$ be the dual of $\mathfrak{H}_{0}^{1}(\sigma(u))$; it is a Hilbert space equipped with the norm $\|\cdot\|_{-1, \sigma(u)}$ defined by

$$
\|L\|_{-1, \sigma(u)}^{2}=\sup _{H}\left\{2\left\langle\langle L, H\rangle-\|H\|_{1, \sigma(u)}^{2}\right\},\right.
$$

where the supremum is carried over all functions $H \in C_{0}^{\infty}([-T, 0] \times[0,1])$, equivalently over all $H \in \mathfrak{H}_{0}^{1}(\sigma(u))$, and $\langle\langle L, H\rangle\rangle$ stands for the value of the linear functional $L$ at $H$. The next statement is proven in [10. A functional $f: X \rightarrow$ $(-\infty,+\infty]$ defined on a Polish space $X$ is said to be coercive if all its sub-level sets are precompact: for all $t \in \mathbb{R},\{x: f(x) \leq t\}$ is precompact.

Theorem 2.1. For each $\varepsilon>0$ and $T>0$ the functional $I_{[-T, 0]}^{\varepsilon}: C([-T, 0] ; M) \rightarrow$ $[0,+\infty]$ is coercive and lower semicontinuous, namely it has compact sub-level sets. Moreover, given $u$ such that $I_{[-T, 0]}^{\varepsilon}(u)<+\infty$, there exists a unique $H=H(u)$ in $\mathfrak{H}_{0}^{1}(\sigma(u))$ such that $u$ is a weak solution to

$$
\left\{\begin{array}{l}
u_{t}+f(u)_{x}=\varepsilon u_{x x}-2 \varepsilon\left(\sigma(u) H_{x}\right)_{x} \\
u(t, 0)=\rho_{0}, \quad u(t, 1)=\rho_{1} .
\end{array}\right.
$$

In this case, the linear functional $L_{u}^{\varepsilon}$, as defined in (2.2), extends to a linear functional on $\mathfrak{H}_{0}^{1}(\sigma(u))$, that we denote by $u_{t}+f(u)_{x}-\varepsilon u_{x x}$, and

$$
I_{[-T, 0]}^{\varepsilon}(u)=\varepsilon\|H\|_{1, \sigma(u)}^{2}=\frac{1}{4 \varepsilon}\left\|u_{t}+f(u)_{x}-\varepsilon u_{x x}\right\|_{-1, \sigma(u)}^{2} .
$$

The quasi-potential. The quasi-potential 23 associated to the family of action functionals $I_{[-T, 0]}^{\varepsilon}, T>0$, is the functional $V_{\varepsilon}: M \rightarrow[0,+\infty]$ defined by

$$
V_{\varepsilon}(\rho):=\inf _{T>0} \inf \left\{I_{[-T, 0]}^{\varepsilon}(u): u \in C([-T, 0] ; M), u(-T)=\bar{\rho}_{\varepsilon}, u(0)=\rho\right\},
$$

so that $V_{\varepsilon}(\rho)$ measures the minimal cost to reach the function $\rho$ starting from the stationary solution $\bar{\rho}_{\varepsilon}$. In this sense, while $I_{[-T, 0]}^{\varepsilon}(u)$ measures how much a path $u$ is close to solutions to (2.1), the quasi-potential $V_{\varepsilon}(\rho)$ measures how much $\rho$ is close to the stationary solution $\bar{\rho}_{\varepsilon}$. 
The previous definition implies that $V_{\varepsilon}$ is a Lyapunov functional for the Burgers equation (2.1). This is to say that if $u(t ; \rho), t \geq 0$, is the solution to (2.1) with initial datum $u(0, \cdot ; \rho)=\rho(\cdot) \in M$ then for any $t \geq 0$ we have $V_{\varepsilon}(u(t ; \rho)) \leq V_{\varepsilon}(\rho)$. Observe that for each $t \geq 0$ we have $u(t ; \rho) \in M$ by the maximum principle. The previous claim is easily proven, recalling that $I_{[-T, 0]}^{\varepsilon}$ vanishes on weak solutions to (2.1), by exhibiting a test path for the variational problem (2.4).

It is convenient to formulate the variational problem (2.4) also on paths defined on the semi-infinite time interval $(-\infty, 0]$. To this end we introduce the set

$$
\mathcal{U}\left(\bar{\rho}_{\varepsilon}\right):=\left\{u \in C((-\infty, 0] ; M): \lim _{t \rightarrow-\infty} u(t)=\bar{\rho}_{\varepsilon}\right\}
$$

equipped with the topology of the uniform convergence. The family of action functionals $I_{[-T, 0]}^{\varepsilon}, T>0$, naturally induces the lower semicontinuous functional $I^{\varepsilon}: \mathcal{U}\left(\bar{\rho}_{\varepsilon}\right) \rightarrow[0,+\infty]$ defined by

$$
I^{\varepsilon}(u):=\lim _{T \rightarrow \infty} I_{[-T, 0]}^{\varepsilon}\left(u \uparrow_{[-T, 0]}\right)
$$

where $u \uparrow_{[-T, 0]}$ denotes the restriction of $u \in \mathcal{U}\left(\bar{\rho}_{\varepsilon}\right)$ to $C([-T, 0] ; M)$. Note that the above limit always exists, possibly equal to $+\infty$, in view of the monotonicity of $I_{[-T, 0]}^{\varepsilon}\left(u \uparrow_{[-T, 0]}\right)$ in $T>0$. Let

$$
\widehat{V}_{\varepsilon}(\rho):=\inf \left\{I^{\varepsilon}(u): u \in \mathcal{U}\left(\bar{\rho}_{\varepsilon}\right), u(0)=\rho\right\}
$$

and observe that the inequality $\widehat{V}_{\varepsilon} \leq V_{\varepsilon}$ holds trivially. In the context of diffusion processes in $\mathbb{R}^{n}$, by the continuity of $\widehat{V}_{\varepsilon}$, it is easy to show that $V_{\varepsilon}=\widehat{V}_{\varepsilon}[23$. We shall prove that this identity also holds in the present setting where, as shown below, $\widehat{V}_{\varepsilon}$ is not continuous but only lower semicontinuous.

Characterization of the quasi-potential. The analysis of the quasi-potential in the case $\rho_{0}>\rho_{1}$ and in the case $\rho_{0}<\rho_{1}, \varepsilon \geq \varepsilon_{0}$ has been considered in [8]. Here we discuss the more interesting case in which $\rho_{0}<\rho_{1}$ and $\varepsilon \in\left(0, \varepsilon_{0}\right)$. The first main result of this article states that the quasi-potential $V_{\varepsilon}$ can be expressed in terms of a static variational problem.

Given $m>0$, denote by $\mathcal{P}_{m}([0,1])$ the set of positive Borel measures on $[0,1]$ with total mass equal to $m$. Recall that $\varphi_{i}=\log \left[\rho_{i} /\left(1-\rho_{i}\right)\right], i=0,1, \varphi_{0}<\varphi_{1}$, and set

$$
\mathcal{F}:=\left\{\varphi: \varphi(x)=\varphi_{0}+\mu([0, x]) \text { for some } \mu \in \mathcal{P}_{\varphi_{1}-\varphi_{0}}([0,1])\right\} .
$$

Clearly, if $\varphi$ belongs to $\mathcal{F}$ then $\varphi$ is an increasing càdlàg function satisfying $\varphi_{0} \leq$ $\varphi(0), \varphi(1)=\varphi_{1}$. We consider the set $\mathcal{F}$ equipped with the topology inherited from the weak convergence of measures, namely a sequence $\left\{\varphi^{n}\right\} \subset \mathcal{F}$ converges to $\varphi$ in $\mathcal{F}$ if and only if for any continuous function $g$ on $[0,1]$ we have $\int g d \varphi^{n} \rightarrow \int g d \varphi$. Then $\mathcal{F}$ is a compact Polish space. Moreover, $\varphi^{n} \rightarrow \varphi$ in $\mathcal{F}$ implies $\varphi^{n} \rightarrow \varphi$ a.e.

Let $s: \mathbb{R} \rightarrow(-\infty,+\infty]$ be the convex function defined by

$$
s(a):= \begin{cases}a \log a+(1-a) \log (1-a) & \text { if } a \in[0,1] \\ +\infty & \text { otherwise }\end{cases}
$$

and observe that for $a \in(0,1)$ we have $s^{\prime \prime}(a) \sigma(a)=1$. Let $\mathcal{G}_{\varepsilon}: M \times \mathcal{F} \rightarrow(-\infty,+\infty]$ be the functional defined by

$$
\mathcal{G}_{\varepsilon}(\rho, \varphi)=\int_{0}^{1}\left[s(\rho)+s\left(\varepsilon \varphi_{x}\right)+(1-\rho) \varphi-\log \left(1+e^{\varphi}\right)\right] d x,
$$


where we understand that $\mathcal{G}_{\varepsilon}(\rho, \varphi)=+\infty$ unless the measure $d \varphi$ is absolutely continuous with respect to the Lebesgue measure and its density, denoted by $\varphi_{x}$, is bounded by $\varepsilon^{-1}$. By the convexity of $s$, the functional $\mathcal{G}_{\varepsilon}$ is lower semicontinuous.

We shall connect the quasi-potential $V_{\varepsilon}(\rho)$ to the minimum of the functional $\mathcal{G}_{\varepsilon}(\rho, \cdot)$ over $\mathcal{F}$. Fix $\rho$ in $M$ and consider the Euler-Lagrange equation associated to the functional $\mathcal{G}_{\varepsilon}(\rho, \cdot)$

$$
\left\{\begin{array}{l}
\frac{\varepsilon \varphi_{x x}}{\varphi_{x}\left(1-\varepsilon \varphi_{x}\right)}-\frac{1}{1+e^{\varphi}}+\rho=0, \\
\varphi(0)=\varphi_{0}, \quad \varphi(1)=\varphi_{1} .
\end{array}\right.
$$

Since this equation is not really meaningful for $\varphi \in \mathcal{F}$, we formulate it as a fixed point condition for a suitable operator. Denote by $K_{\rho, \varepsilon}=K\left(\varepsilon, \varphi_{0}, \varphi_{1}, \rho\right): \mathcal{F} \rightarrow \mathcal{F}$ the integral operator

$$
\left(K_{\rho, \varepsilon} \varphi\right)(x)=\varphi_{0}+\frac{1}{\varepsilon} \int_{0}^{x} \frac{A \exp \left\{\frac{1}{\varepsilon} \int_{0}^{y}\left[\left(1+e^{\varphi}\right)^{-1}-\rho\right] d z\right\}}{1+A \exp \left\{\frac{1}{\varepsilon} \int_{0}^{y}\left[\left(1+e^{\varphi}\right)^{-1}-\rho\right] d z\right\}} d y,
$$

where $A=A(\varepsilon, \rho, \varphi) \in(0, \infty)$ is chosen so that $\left(K_{\rho, \varepsilon} \varphi\right)(1)=\varphi_{1}$. We prove in Section 4 that such a choice is always possible and unique. Moreover, if $\varphi$ is a fixed point of $K_{\rho, \varepsilon}$, then $\varphi$ is in $C^{1}([0,1])$, it has a Lipschitz derivative $\varphi_{x}$ satisfying $0<\varepsilon \varphi_{x}<1$, and $\varphi$ solves (2.11) a.e. We adopt, in particular, the following terminology. A function $\varphi \in \mathcal{F}$ is said to be a solution of (2.11) if it belongs to $\mathcal{P}_{\varepsilon}(\rho)$, the set of fixed points of $K_{\rho, \varepsilon}$.

Recall that $\bar{\rho}_{\varepsilon}$ is the stationary solution to (2.1). Let $S_{\varepsilon}^{o}, S_{\varepsilon}: M \rightarrow \mathbb{R}$ be the functionals defined by

$$
S_{\varepsilon}^{o}(\rho):=\inf \left\{\mathcal{G}_{\varepsilon}(\rho, \varphi), \varphi \in \mathcal{F}\right\}, \quad S_{\varepsilon}(\rho)=S_{\varepsilon}^{o}(\rho)-S_{\varepsilon}^{o}\left(\bar{\rho}_{\varepsilon}\right)
$$

and note that the infimum is achieved by the lower semicontinuity of $\mathcal{G}_{\varepsilon}(\rho, \cdot)$ and by the compactness of $\mathcal{F}$. Given $\rho \in M$, we denote by $\mathcal{F}_{\varepsilon}(\rho) \subset \mathcal{F}$ the collection of minimizers for the previous variational problem, i.e.

$$
\mathcal{F}_{\varepsilon}(\rho):=\arg \inf \left\{\mathcal{G}_{\varepsilon}(\rho, \varphi), \varphi \in \mathcal{F}\right\}
$$

and observe that $\mathcal{F}_{\varepsilon}(\rho)$ is a non-empty compact subset of $\mathcal{F}$.

Theorem 2.2. Fix $\varphi_{0}<\varphi_{1}, \varepsilon \in\left(0, \varepsilon_{0}\right)$, and $\rho \in M$. Then any minimizer of $\mathcal{G}_{\varepsilon}(\rho, \cdot)$ solves (2.11), i.e.

$$
\mathcal{F}_{\varepsilon}(\rho) \subset \mathcal{P}_{\varepsilon}(\rho) \text {. }
$$

Moreover, the functional $S_{\varepsilon}$ is lower semicontinuous on $M$. Finally, if the sequence $\left\{\rho^{n}\right\} \subset M$ converges to $\rho$ strongly in $L^{1}([0,1])$ then $S_{\varepsilon}\left(\rho^{n}\right) \rightarrow S_{\varepsilon}(\rho)$.

The connection between the quasi-potential and the functional (2.13) is established by the following theorem, which is the main result of this paper.

Theorem 2.3. For each $\rho_{0}<\rho_{1}$ and $\varepsilon \in\left(0, \varepsilon_{0}\right)$ we have $V_{\varepsilon}=\widehat{V}_{\varepsilon}=S_{\varepsilon}$.

We remark that while the identity $V_{\varepsilon}=\widehat{V}_{\varepsilon}$ holds under general conditions, the characterization of the quasi-potential in terms of the static variational problem (2.13) depends crucially on the specific form of the flux $f$ and the mobility $\sigma$ namely, $f(a)=\sigma(a)=a(1-a), a \in[0,1]$.

In the proof of Theorem 2.3 we actually describe some optimal paths for the variational problem (2.7). Fix $\rho$ in $M$, let $\varphi \in \mathcal{F}_{\varepsilon}(\rho)$, and denote by $F=F(t, x)$, 
$(t, x) \in[0,+\infty) \times[0,1]$ the solution of the viscous Burgers equation (2.1) with initial condition $e^{\varphi} /\left(1+e^{\varphi}\right)$. Set $\psi=s^{\prime}(F)$ and define $v=v(t, x)$ by

$$
v=\frac{1}{1+e^{\psi}}-\frac{\varepsilon \psi_{x x}}{\psi_{x}\left(1-\varepsilon \psi_{x}\right)} .
$$

We prove that an optimal path $u$ for the variational problem (2.7) is the path $v$ reversed in time, i.e. $u(t)=v(-t)$. This construction shows that to each $\varphi \in \mathcal{F}_{\varepsilon}(\rho)$ there is associated a path $u \in \mathcal{U}\left(\bar{\rho}_{\varepsilon}\right)$ which is a minimizer for the variational problem (2.7). If $\mathcal{F}_{\varepsilon}(\rho)$ is not a singleton, to different elements in $\mathcal{F}_{\varepsilon}(\rho)$ are associated different minimizers for (2.7) and there is no uniqueness of the minimizer for (2.7). Unfortunately, we are not able to prove that any minimizer of (2.7) can be obtained from the previous construction; in particular we cannot deduce uniqueness of the minimizer for (2.7) from the uniqueness of the minimizer for $\mathcal{G}_{\varepsilon}(\rho, \cdot)$. We refer however to the heuristic argument presented below, in the context of the Hamiltonian formalism, which suggests that the minimizers for (2.7) are indeed in a one-to-one correspondence with $\mathcal{F}_{\varepsilon}(\rho)$.

Theorem 2.3 implies that the minimum of $S_{\varepsilon}^{o}$ is achieved at $\bar{\rho}_{\varepsilon}$, equivalently that $S_{\varepsilon}$ is a positive functional. This can also be shown by direct computations. Indeed it is enough to observe that, for a fixed $\varphi \in \mathcal{F}$, the strict convexity of the map $\rho \mapsto \mathcal{G}_{\varepsilon}(\rho, \varphi)$ implies that the infimum over $\rho$ is uniquely attained for $\varphi=s^{\prime}(\rho)$. A straightforward computation then shows that the functional $\varphi \mapsto \mathcal{G}_{\varepsilon}\left(s^{\prime-1}(\varphi), \varphi\right)$ has a unique critical point, which is a global minimum, achieved at $s^{\prime}\left(\bar{\rho}_{\varepsilon}\right)$.

Uniqueness / non uniqueness of optimal paths. The connection between the quasi-potential $V_{\varepsilon}$ and a "trial" functional like $\mathcal{G}_{\varepsilon}$ has been established for other action functionals arising as large deviation rate functional for few microscopic stochastic dynamics in the diffusive scaling limit 6, 8, 16, 20. In contrast with all other cases, the functional $\mathcal{G}_{\varepsilon}(\rho, \cdot)$ is neither concave nor convex and might have more than a single critical point. Fix $\rho \in M$. The construction presented above actually shows that to each critical point $\varphi$ for $\mathcal{G}_{\varepsilon}(\rho, \cdot)$ there is associated a path $u \in \mathcal{U}\left(\bar{\rho}_{\varepsilon}\right)$ which is a critical point for the variational problem (2.7). It is therefore natural to investigate whether the sets $\mathcal{P}_{\mathcal{\varepsilon}}(\rho)$ and $\mathcal{F}_{\varepsilon}(\rho)$ are singletons.

In this direction, our first result shows that - under suitable conditions - there exists a unique critical point for $\mathcal{G}_{\varepsilon}(\rho, \cdot)$.

Theorem 2.4. The following statements hold.

(i) Fix $\varphi_{0}<\varphi_{1}$. There exists $\varepsilon_{1} \in\left(0, \varepsilon_{0}\right)$ such that for any $\varepsilon \in\left(\varepsilon_{1}, \varepsilon_{0}\right)$ the set $\mathcal{P}_{\varepsilon}(\rho)$ is a singleton for any $\rho \in M$.

(ii) Fix $\varepsilon>0$. There exists $\delta \in\left(0, \varepsilon^{-1}\right)$ such that for any $0<\varphi_{1}-\varphi_{0}<\delta$ the set $\mathcal{P}_{\varepsilon}(\rho)$ is a singleton for any $\rho \in M$.

(iii) Fix $\varphi_{0}<\varphi_{1}$ and $\varepsilon \in\left(0, \varepsilon_{0}\right)$. If $\rho \in M$ is in $C^{1}([0,1])$ and strictly increasing then the set $\mathcal{P}_{\varepsilon}(\rho)$ is a singleton.

We remark that while the first two results are based on a standard perturbation argument and are quite natural from a statistical mechanics point of view, the third one somehow depends on the global structure of the functional $\mathcal{G}_{\varepsilon}$. Recalling that the stationary solution $\bar{\rho}_{\varepsilon}$ is smooth and strictly increasing, the third statement implies that the functional $\mathcal{G}_{\varepsilon}(\rho, \cdot)$ has a unique critical point when $\rho$ lies in a $C^{1}$-neighborhood of $\bar{\rho}_{\varepsilon}$. 
As discussed above, a most striking feature of the model here examined is that there can be more than a single minimizer for $\mathcal{G}_{\varepsilon}(\rho, \cdot)$. The next result states that this phenomenon does indeed occur.

Theorem 2.5. Fix $\varphi_{0}<\varphi_{1}$. There exists $\varepsilon_{2} \in\left(0, \varepsilon_{0}\right)$ such that the following statement holds. For each $\varepsilon \in\left(0, \varepsilon_{2}\right)$ there exist functions $\rho \in M$ such that $\mathcal{F}_{\varepsilon}(\rho)$ is not a singleton.

As we show in Proposition 4.6, at the points $\rho$ where $\mathcal{F}_{\varepsilon}(\rho)$ is not a singleton the quasi-potential admits more than one Gâteaux super-differential. The proof of the above theorem is based on a perturbation argument with respect to the limiting case $\varepsilon=0$ that we next discuss.

The inviscid limit. It is well known, see e.g. [34, Ch. 15], that in the inviscid limit $\varepsilon \downarrow 0$, the solution to the Cauchy problem associated to 2.1) converges to the entropy solution of the Cauchy problem associated to the inviscid Burgers equation $u_{t}+f(u)_{x}=0$ with the Bardos-Leroux-Nédélec boundary conditions [2]. We also mention that in the case of the Burgers equation on $\mathbb{R}$, the variational convergence as $\varepsilon \downarrow 0$ of the action functional $I_{[-T, 0]}^{\varepsilon}$ for a fixed $T>0$ is discussed in [3]. Referring to [8] for the case $\rho_{0}>\rho_{1}$, we here discuss the variational convergence of the quasi-potential $V_{\varepsilon}$ as $\varepsilon \downarrow 0$.

In the inviscid limit $\varepsilon=0$, the stationary solutions $\bar{\rho}$ are easily described by considering the propagation of shocks for the inviscid Burgers equation on $\mathbb{R}$. If $1-\left(\rho_{1}+\rho_{0}\right)>0$ an entropic shock from $\rho_{0}$ to $\rho_{1}$ travels to the right so that $\bar{\rho}=\rho_{0}$, while $\bar{\rho}=\rho_{1}$ if $1-\left(\rho_{0}+\rho_{1}\right)<0$. In the case $1-\left(\rho_{0}+\rho_{1}\right)=0$ there is a one parameter family of stationary entropic solutions which corresponds to a stationary shock that can be placed anywhere in [0,1]. Equivalently, a stationary entropic solution $\bar{\rho}$ satisfies $f(\bar{\rho})=\min _{r \in\left[\rho_{0}, \rho_{1}\right]} f(r)$. For $1-\left(\rho_{0}+\rho_{1}\right) \neq 0$ it is not difficult to check that, as $\varepsilon \downarrow 0$, the stationary solution $\bar{\rho}_{\varepsilon}$ converges strongly in $L^{1}([0,1])$ to the constant function equal to $\bar{\rho}$. In the case $1-\left(\rho_{0}+\rho_{1}\right)=0, \bar{\rho}_{\varepsilon}$ converges to the stationary solution of the inviscid Burgers equation with a shock placed at $x=1 / 2$.

Recall 2.10) and let $\mathcal{G}: M \times \mathcal{F} \rightarrow \mathbb{R}$ be the lower semicontinuous functional defined by

$$
\mathcal{G}(\rho, \varphi):=\int_{0}^{1}\left[s(\rho)+(1-\rho) \varphi-\log \left(1+e^{\varphi}\right)\right] d x .
$$

Let also $S^{o}, S: M \rightarrow \mathbb{R}$ be the functionals defined by

$$
S^{o}(\rho):=\inf \{\mathcal{G}(\rho, \varphi), \varphi \in \mathcal{F}\}, \quad S(\rho):=S^{o}(\rho)-S^{o}(\bar{\rho})
$$

and observe the infimum is achieved by the lower semicontinuity of $\mathcal{G}(\rho, \cdot)$ and the compactness of $\mathcal{F}$. As discussed before, for $\rho_{0}+\rho_{1} \neq 1$ there exists a unique stationary entropic solution $\bar{\rho}$ of the inviscid Burgers equation. On the other hand, if $\rho_{0}+\rho_{1}=1$ there exists a one-parameter family of stationary entropic solutions $\left\{\bar{\rho}_{\alpha}, \alpha \in[0,1]\right\}$; it is however simple to check that $S^{o}\left(\bar{\rho}_{\alpha}\right)$ is in fact independent of $\alpha$. This shows the functional $S$ is well defined.

As we show in Proposition 6.3 below, we may restrict the infimum in (2.16) to functions $\varphi \in \mathcal{F}$ which are step functions in the sense that $\varphi$ jumps from $\varphi_{0}$ to $\varphi_{1}$ at a single point in $[0,1]$. In view of this result, simple computations show that $S$ coincides with the functional derived in [17] within the context of the boundary driven asymmetric exclusion process. 
In the inviscid limit $\varepsilon \downarrow 0$ we expect the functional $S_{\varepsilon}$ to converge to $S$. From a variational point of view, the natural notion of convergence is the so-called $\Gamma$ convergence that we next recall, see e.g. [12. Let $X$ be a Polish space. A sequence of functionals $F_{n}: X \rightarrow(-\infty,+\infty]$ is said to $\Gamma$-converge to a functional $F$ : $X \rightarrow(\infty,+\infty]$ if the following two conditions hold for each $x \in X$. There exists a sequence $x_{n} \rightarrow x$ such that $\limsup _{n} F_{n}\left(x_{n}\right) \leq F(x)$ ( $\Gamma$-limsup inequality) and for any sequence $x_{n} \rightarrow x$ we have $\liminf { }_{n} F_{n}\left(x_{n}\right) \geq F(x)$ ( $\Gamma$-liminf inequality).

Theorem 2.6. Let $S_{\varepsilon}, S: M \rightarrow[0, \infty)$ be defined as in (2.13), (2.16), respectively. The family of functionals $\left\{S_{\varepsilon}\right\}_{\varepsilon>0} \Gamma$-converges to $S$ in $M$ as $\varepsilon \downarrow 0$. In particular, the functional $S: M \rightarrow[0,+\infty)$ is lower semicontinuous.

Given $\rho \in M$, we also expect that the minimizers of $\mathcal{G}_{\varepsilon}(\rho, \cdot)$ converge, as $\varepsilon \downarrow 0$, to a minimizer of $\mathcal{G}(\rho, \cdot)$. The precise statement is the following.

Theorem 2.7. Fix $\rho \in M$ and let $\varphi_{\varepsilon} \in \mathcal{F}_{\varepsilon}(\rho)$. If $\varepsilon_{n} \downarrow 0$ is a sequence such that $\varphi_{\varepsilon_{n}} \rightarrow \varphi$ for some $\varphi \in \mathcal{F}$, then $\varphi$ is a minimizer for $\mathcal{G}(\rho, \cdot)$.

Notation warning. Apart when we discuss, in Section 6. the inviscid limit, the parameter $\varepsilon>0$ is kept fixed. We therefore drop from most of the notation the explicit dependence of the functionals on $\varepsilon$.

\section{Hamiltonian picture}

As it will be clear in the following discussion, the variational problem (2.7) is naturally described within the Hamiltonian formalism. Accordingly, Theorem 2.3 reflects a peculiar geometric structure of the underlying phase space. In this section we present heuristically this picture, but we emphasize that the actual proofs are logically independent from it.

The functional $I_{[-T, 0]}$ in (2.3) is the action functional corresponding to the Lagrangian

$$
\mathbb{L}\left(u, u_{t}\right)=\frac{1}{4 \varepsilon}\left|u_{t}+f(u)_{x}-\varepsilon u_{x x}\right|_{-1, \sigma(u)}^{2} .
$$

The associated Hamiltonian, obtained by a Legendre transform, is given by

$$
\mathbb{H}(\rho, h)=\varepsilon\left\langle h_{x}, \sigma(\rho) h_{x}\right\rangle+\left\langle\varepsilon \rho_{x x}-f(\rho)_{x}, h\right\rangle,
$$

where $\rho:[0,1] \rightarrow[0,1]$ satisfies $\rho(0)=\rho_{0}, \rho(1)=\rho_{1}$, and $h:[0,1] \rightarrow \mathbb{R}$ is the momentum, satisfying the boundary conditions $h(0)=h(1)=0$. The canonical equations associated to the Hamiltonian $\mathbb{H}$ are

$$
\left\{\begin{array}{l}
u_{t}=\varepsilon u_{x x}-f(u)_{x}-2 \varepsilon\left(\sigma(u) H_{x}\right)_{x} \\
H_{t}=-\varepsilon H_{x x}-f^{\prime}(u) H_{x}-\varepsilon \sigma^{\prime}(u) H_{x}^{2}
\end{array}\right.
$$

with the boundary conditions $u(t, 0)=\rho_{0}, u(t, 1)=\rho_{1}$ and $H(t, 0)=H(t, 1)=0$. It is not clear whether the above equations do define, even locally, a flow and the discussion will be here kept at the informal level.

As follows from the exponential attractiveness of $\bar{\rho}_{\varepsilon}$ for the flow defined by (2.1), $\left(\bar{\rho}_{\varepsilon}, 0\right)$ is a hyperbolic fixed point of the Hamilton flow (3.2). Denote by $\mathcal{M}_{\mathrm{s}}, \mathcal{M}_{\mathrm{u}}$ the associated stable and unstable manifolds. Of course, $\mathcal{M}_{\mathrm{s}}, \mathcal{M}_{u} \subset\{(\rho, h): \mathbb{H}(\rho, h)=$ $\left.\mathbb{H}\left(\bar{\rho}_{\varepsilon}, 0\right)=0\right\}$. Each point $(\rho, 0)$ is driven by the flow to $\left(\bar{\rho}_{\varepsilon}, 0\right)$ as $t \rightarrow+\infty$, and therefore $\mathcal{M}_{\mathrm{s}} \supset\{(\rho, h): h=0\}$. 
Recall the Poincaré-Cartan theorem, see e.g. 11 § 44], which states that the integral of the symplectic one-form $\langle h, d \rho\rangle$ along any closed path in the phase space is preserved by the Hamiltonian flow. This implies that the stable and unstable manifolds are Lagrangian, namely for any closed path which lies either in $\mathcal{M}_{s}$ or in $\mathcal{M}_{\mathrm{u}}$,

$$
\oint\langle H, d u\rangle=0 \text {. }
$$

We can therefore define the pre-potential $W_{\varepsilon}: \mathcal{M}_{\mathrm{u}} \rightarrow \mathbb{R}$ by

$$
W_{\varepsilon}(\rho, h)=\int_{\Gamma}\langle H, d u\rangle,
$$

where the integral is carried over a path $\Gamma$ in $\mathcal{M}_{\mathbf{u}}$ which connects $\left(\bar{\rho}_{\varepsilon}, 0\right)$ to $(\rho, h)$. Recalling (2.7), the connection between the quasi-potential and the pre-potential is given by

$$
\widehat{V}_{\varepsilon}(\rho)=\inf \left\{W_{\varepsilon}(\rho, h), h:(\rho, h) \in \mathcal{M}_{\mathrm{u}}\right\}
$$

In a finite dimensional framework, this result is proven in [13, 14]. For the reader's convenience, we sketch the basic argument.

Denote by $U_{\varepsilon}(\rho)$ the right hand side of (3.4). By means of compactness arguments, one shows the existence of a path $u \in \mathcal{U}\left(\bar{\rho}_{\varepsilon}\right)$ satisfying $u(0)=\rho$ and such that

$$
\widehat{V}_{\varepsilon}(\rho)=I(u)=\int_{-\infty}^{0} \mathbb{L}\left(u, u_{t}\right) d t .
$$

Since $u$ is an extremal path, it satisfies the Euler-Lagrange equation, or, equivalently, the pair $(u, H)$, where $H=\delta \mathbb{L} / \delta u_{t}$ stands for the conjugate momentum, solves the canonical equations (3.2). One then shows that the trajectory $(u, H)$ lies in the unstable manifold $\mathcal{M}_{\mathrm{u}}$; while $u(t) \rightarrow \bar{\rho}_{\varepsilon}$ as $t \rightarrow-\infty$ follows from the definition, some efforts are required to show that also $H(t) \rightarrow 0$ as $t \rightarrow-\infty$. By Legendre duality, the inclusion $\mathcal{M}_{\mathrm{u}} \subset\{\mathbb{H}=0\}$, and (3.3.

$$
\begin{aligned}
\widehat{V}_{\varepsilon}(\rho) & =I(u)=\int_{-\infty}^{0} \mathbb{L}\left(u, u_{t}\right) d t=\int_{-\infty}^{0}\left[\left\langle H, u_{t}\right\rangle-\mathbb{H}(u, H)\right] d t \\
& =\int_{-\infty}^{0}\left\langle H, u_{t}\right\rangle d t=W_{\varepsilon}(\rho, H(0)) \geq U_{\varepsilon}(\rho) .
\end{aligned}
$$

The proof of the reverse inequality is simple. Fix $\rho$ and choose $h$ which minimizes the right hand side of (3.4). Since $(\rho, h)$ belongs to $\mathcal{M}_{\mathrm{u}}$, we need only to follow the Hamiltonian flow (3.2) to obtain a path $(u, H)$ such that $u(0)=\rho, H(0)=h$ and $(u(t), H(t)) \rightarrow\left(\bar{\rho}_{\varepsilon}, 0\right)$ as $t \rightarrow-\infty$. The previous computations now give

$$
\widehat{V}_{\varepsilon}(\rho) \leq I(u)=W_{\varepsilon}(\rho, h)=U_{\varepsilon}(\rho) .
$$

The above argument actually shows that any minimizer $u$ for the variational problem (2.7) is a solution to the canonical equations (3.2) with $u(0)=\rho$ and $H(0)=h$ where $h$ is a minimizer for the right hand side of (3.4).

In a neighborhood of the fixed point $\left(\bar{\rho}_{\varepsilon}, 0\right)$, the unstable manifold $\mathcal{M}_{\mathrm{u}}$ can be written as a graph, namely it has the form $\mathcal{M}_{\mathrm{u}}=\left\{(\rho, h): h=m_{\mathrm{u}}(\rho)\right\}$ for some map $m_{\mathrm{u}}$. In this case, the infimum on the right hand side of (3.4) is trivial and $\widehat{V}_{\varepsilon}(\rho)=W_{\varepsilon}\left(\rho, m_{\mathrm{u}}(\rho)\right)$. In general, though, this is not true globally. Given $\rho \in M$, to each $h$ such that $(\rho, h) \in \mathcal{M}_{\mathrm{u}}$, there corresponds a critical point for the variational problem (2.7). It may happen, for special $\rho$, that the variational problem on the 
right hand side of (3.4) admits more than a single minimizer. In this case there is also more than one minimizer for the variational problem (2.7). In particular, as will be clearer in the following, Theorem 2.4 implies that, for either $\varepsilon$ close to $\varepsilon_{0}$ or $\varphi_{1}-\varphi_{0}$ small, the manifold $\mathcal{M}_{\mathrm{u}}$ is globally a graph. On the other hand, when $\mathcal{P}(\rho)$ is not a singleton for some $\rho \in M$ the manifold $\mathcal{M}_{\mathrm{u}}$ is not globally a graph. Finally, Theorem 2.5 implies that for $\varepsilon$ small enough there exist functions $\rho$ such that the minimizer for the right hand side of (3.4) is not unique.

In view of (3.4), to prove heuristically Theorem 2.3 we need to replace $W_{\varepsilon}$ by $\mathcal{G}_{\varepsilon}$ on the right hand side of (3.4). It is convenient to perform the symplectic change of variables $(\rho, h) \mapsto(\varphi, \pi)$ given by

$$
\left\{\begin{array}{l}
\varphi=s^{\prime}(\rho)-h, \\
\pi=\rho .
\end{array}\right.
$$

In the new variables $(\varphi, \pi)$ the Hamiltonian reads

$$
\widetilde{\mathbb{H}}(\varphi, \pi)=\mathbb{H}\left(\pi, s^{\prime}(\pi)-\varphi\right)=\varepsilon\left\langle\varphi_{x}, \sigma(\pi) \varphi_{x}\right\rangle-\left\langle\varepsilon \pi_{x}+\sigma(\pi), \varphi_{x}\right\rangle+\rho_{1}-\rho_{0},
$$

where we used that $s^{\prime}(\pi)-\varphi$ vanishes at the boundary, $\sigma(\pi) s^{\prime \prime}(\pi)=1, f=\sigma$ and $\rho(0)=\rho_{0}, \rho(1)=\rho_{1}$. The corresponding canonical equations are

$$
\left\{\begin{array}{l}
\Phi_{t}=\frac{\delta \widetilde{\mathbb{H}}}{\delta \Pi}=\varepsilon \Phi_{x x}-\sigma^{\prime}(\Pi) \Phi_{x}\left(1-\varepsilon \Phi_{x}\right), \\
\Pi_{t}=-\frac{\delta \widetilde{\mathbb{H}}}{\delta \Phi}=-\varepsilon \Pi_{x x}-\sigma(\Pi)_{x}+2 \varepsilon\left(\sigma(\Pi) \Phi_{x}\right)_{x} .
\end{array}\right.
$$

In the new variables the fixed point $\left(\bar{\rho}_{\varepsilon}, 0\right)$ reads $\left(s^{\prime}\left(\bar{\rho}_{\varepsilon}\right), \bar{\rho}_{\varepsilon}\right)$. The associated stable manifold is $\left\{(\varphi, \pi): \varphi=s^{\prime}(\pi)\right\}$. Let

$$
\Sigma=\left\{(\varphi, \pi): \pi=\frac{1}{1+e^{\varphi}}-\frac{\varepsilon \varphi_{x x}}{\varphi_{x}\left(1-\varepsilon \varphi_{x}\right)}\right\} .
$$

By using that $\sigma(\pi)=\pi(1-\pi)$, a long and tedious computation that we omit shows that the set $\Sigma$ is invariant under the flow (3.7). More precisely, pick a point $(\varphi, \pi) \in \Sigma$ and let $\Phi$ be the solution to

$$
\Phi_{t}=-\varepsilon \Phi_{x x}+\frac{1-e^{\Phi}}{1+e^{\Phi}} \Phi_{x}\left(1-\varepsilon \Phi_{x}\right)
$$

with initial condition $\Phi(0)=\varphi$. Set now

$$
\Pi=\frac{1}{1+e^{\Phi}}-\frac{\varepsilon \Phi_{x x}}{\Phi_{x}\left(1-\varepsilon \Phi_{x}\right)}
$$

and observe that $\Pi(0)=\pi$ since $(\varphi, \pi) \in \Sigma$. Then $(\Phi, \Pi)$ is a solution to the canonical equations (3.7). Moreover, as we show in Lemmata 5.4 and 5.5, under the Hamiltonian flow any point in $\Sigma$ converges to the fixed point $\left.\left(s^{\prime}\left(\bar{\rho}_{\varepsilon}\right), \bar{\rho}_{\varepsilon}\right)\right)$ as $t \rightarrow-\infty$. This implies $\Sigma$ is the unstable manifold. The previous arguments really only show that $\left\{(\varphi, \pi): \varphi=s^{\prime}(\pi)\right\} \subset \mathcal{M}_{\mathrm{s}}$ and $\Sigma \subset \mathcal{M}_{\mathrm{u}}$. On the other hand, as the tangent spaces to $\left\{(\varphi, \pi): \varphi=s^{\prime}(\pi)\right\}$ and $\Sigma$ at $\left(s^{\prime}\left(\bar{\rho}_{\varepsilon}\right), \bar{\rho}_{\varepsilon}\right)$ span the whole space, we informally claim that the previous inclusions are equalities.

At this point, the informal deduction of Theorem 2.3 will be completed by the computation of the pre-potential. This is easily achieved in the new variables $(\varphi, \pi)$. 
We start by the generating function of the symplectic transformation (3.5). Let

$$
F(\rho, \varphi)=\int_{0}^{1}[s(\rho)-\rho \varphi] d x
$$

be the so-called free generating function of (3.5) (see e.g. [1, $\S 48]$ ), so that

Equivalently,

$$
h=\frac{\delta F}{\delta \rho}, \quad \pi=-\frac{\delta F}{\delta \varphi} .
$$

$$
\langle h, d \rho\rangle-\langle\pi, d \varphi\rangle=d F .
$$

Hence, for any path $\Gamma=\{\gamma(t), t \in[0,1]\}$ in the phase space

$$
\int_{\Gamma}\langle H, d \Pi\rangle=\int_{\Gamma}\langle\Pi, d \Phi\rangle+F(\gamma(1))-F(\gamma(0)) .
$$

Assume now that $\Gamma \subset \Sigma$. By (3.8) and since

we have that

$$
\frac{\varepsilon \varphi_{x x}}{\varphi_{x}\left(1-\varepsilon \varphi_{x}\right)}=\varepsilon\left[s^{\prime}\left(\varepsilon \varphi_{x}\right)\right]_{x},
$$

$$
\int_{\Gamma}\langle\Pi, d \Phi\rangle=\left.\int_{0}^{1}\left[\Phi(t)-\log \left(1+e^{\Phi(t)}\right)+s\left(\varepsilon \Phi_{x}(t)\right)\right] d x\right|_{t=0} ^{t=1} .
$$

Therefore, in view of (2.10), the previous identities imply that

$$
\int_{\Gamma}\langle H, d u\rangle=\int_{\Gamma}\langle H, d \Pi\rangle=\mathcal{G}_{\varepsilon}(\Pi(1), \Phi(1))-\mathcal{G}_{\varepsilon}(\Pi(0), \Phi(0)) .
$$

Hence, by (3.3), $W_{\varepsilon}(\rho, h)=\mathcal{G}_{\varepsilon}(\rho, \varphi)-\mathcal{G}_{\varepsilon}\left(\bar{\rho}_{\varepsilon}, s^{\prime}\left(\bar{\rho}_{\varepsilon}\right)\right)$, where $h$ and $\varphi$ are related by (3.5). As stated above, $S_{\varepsilon}^{o}\left(\bar{\rho}_{\varepsilon}\right)=\mathcal{G}_{\varepsilon}\left(\bar{\rho}_{\varepsilon}, s^{\prime}\left(\bar{\rho}_{\varepsilon}\right)\right)$ and therefore, in view of (3.4),

$$
\widehat{V}_{\varepsilon}(\rho)=\inf \left\{\mathcal{G}_{\varepsilon}(\rho, \varphi), \varphi:(\varphi, \rho) \in \Sigma\right\}-S_{\varepsilon}^{o}\left(\bar{\rho}_{\varepsilon}\right) .
$$

Since, by Theorem 2.2. $\Sigma$ is the set of critical points of the functional $\mathcal{G}_{\varepsilon}(\pi, \cdot)$, we can drop in the previous formula the condition that $(\varphi, \rho)$ belongs to the unstable manifold $\Sigma$ as this condition will be automatically satisfied by any minimizer. This concludes the heuristic proof of Theorem 2.3 .

\section{The Static VARiational PROBLEM}

In this section we analyze the variational problem (2.13). In particular, we prove here Theorems 2.2 and 2.4 .

Critical points of $\mathcal{G}_{\varepsilon}(\rho, \cdot)$. Fix $\rho \in M$. Recall the definition (2.12) of the operator $K_{\rho}: \mathcal{F} \rightarrow \mathcal{F}$ and that we denote by $\mathcal{P}(\rho) \subset \mathcal{F}$ the set of fixed points of $K_{\rho}$. We claim that there exists a unique $A=A(\varphi) \in(0, \infty)$, depending also on $\varphi_{0}, \varphi_{1}, \varepsilon, \rho$, such that $\left(K_{\rho} \varphi\right)(1)=\varphi_{1}$. Note indeed that the integral on the right hand side of (2.12) evaluated for $x=1$ is strictly increasing in $A$, equals 0 when $A$ vanishes, and increases to 1 as $A \uparrow \infty$. Since $\varepsilon\left(\varphi_{1}-\varphi_{0}\right)=\varepsilon / \varepsilon_{0} \in(0,1)$ by assumption, the claim follows. By using that $0 \leq \rho \leq 1$ and $\varphi_{0} \leq \varphi \leq \varphi_{1}$, it is also straightforward to check that there exist reals $0<b_{0}<b_{1}<\infty, b_{i}=b_{i}\left(\varphi_{0}, \varphi_{1}, \varepsilon\right)$, such that for any $\rho \in M$ and $\varphi \in \mathcal{F}$ we have $b_{0} \leq A \leq b_{1}$.

Let $M^{o}$ be the subset of $M$ given by the smooth functions bounded away from zero and one and satisfying the boundary conditions $\rho(0)=\rho_{0}, \rho(1)=\rho_{1}$ :

$$
M^{o}:=\left\{\rho \in C^{2}([0,1]): 0<\rho<1, \rho(0)=\rho_{0}, \rho(1)=\rho_{1}\right\} .
$$


Recall the notation $\mathcal{H}_{0}^{1}(\gamma)$ introduced before Theorem 2.1 and note that for $\rho \in M^{o}$ the norm in $\mathcal{H}_{0}^{1}(\sigma(\rho))$ is in fact equivalent to the norm in the standard Sobolev space $\mathcal{H}_{0}^{1}$. Let the Hamiltonian $\mathbb{H}: M^{o} \times \mathcal{H}_{0}^{1} \rightarrow \mathbb{R}$ be the functional defined in (3.1):

$$
\mathbb{H}(\rho, h):=\varepsilon\left\langle h_{x}, \sigma(\rho) h_{x}\right\rangle-\left\langle\varepsilon \rho_{x}-f(\rho), h_{x}\right\rangle .
$$

Proposition 4.1. Fix $\varphi_{0}<\varphi_{1}, \varepsilon \in\left(0, \varepsilon_{0}\right)$, and $\rho \in M$.

(i) The set $\mathcal{P}(\rho)$ is not empty.

(ii) If $\varphi \in \mathcal{P}(\rho)$ then $\varphi \in C^{1}([0,1])$. Moreover, there exist $\delta \in(0,1 / 2)$ and $C \in$ $(0, \infty)$ independent of $\rho$ such that any $\varphi \in \mathcal{P}(\rho)$ satisfies $\delta \leq \varepsilon \varphi_{x} \leq 1-\delta$, and

$$
\left|\varphi_{x}(x)-\varphi_{x}(y)\right| \leq C|x-y| \quad \forall x, y \in[0,1]
$$

Finally, if $\varphi \in \mathcal{P}(\rho)$ then it solves (2.11) a.e.

(iii) If $\rho \in M^{o}$ then any $\varphi \in \mathcal{P}(\rho)$ belongs to $C^{2}([0,1])$ and satisfies $\mathbb{H}\left(\rho, s^{\prime}(\rho)-\right.$ $\varphi)=0$.

Proof.

(i) It is simple to check that $K_{\rho}: \mathcal{F} \rightarrow \mathcal{F}$ is continuous. By the convexity and compactness of $\mathcal{F}$, the statement follows from Schauder's fixed point theorem.

(ii) Let $\varphi \in \mathcal{P}(\rho)$. The statement $\varphi \in C^{1}([0,1])$ follows immediately from the definition of $K_{\rho}$. By using that $0<b_{0} \leq A \leq b_{1}<\infty$, it is simple to check there exists $\delta>0$ such that $\delta \leq \varepsilon \varphi_{x} \leq 1-\delta$ as well as the Lipschitz bound on $\varphi_{x}$. In view of these bounds, we can rewrite the equation $K_{\rho} \varphi=\varphi$ as

$$
\log \frac{\varepsilon \varphi_{x}(x)}{1-\varepsilon \varphi_{x}(x)}=\log A+\frac{1}{\varepsilon} \int_{0}^{x}\left[\left(1+e^{\varphi(y)}\right)^{-1}-\rho(y)\right] d y .
$$

Since $\varphi_{x}$ is a.e. differentiable, the above identity implies that $\varphi$ satisfies the differential equation in (2.11) a.e.

(iii) The first statement is trivial. To prove the second, observe that if $\rho \in M^{o}$ then $s^{\prime}(\rho)-\varphi$ vanishes at the boundary. Recalling that $f=\sigma$, an integration by parts shows that $\mathbb{H}\left(\rho, s^{\prime}(\rho)-\varphi\right)=0$ is equivalent to

$$
\left\langle\rho_{x},\left(1-\varepsilon \varphi_{x}\right)\right\rangle-\left\langle\sigma(\rho), \varphi_{x}\left(1-\varepsilon \varphi_{x}\right)\right\rangle=0 .
$$

To eliminate from this equation any derivative of $\rho$, we need to integrate by parts the first term. To avoid boundary terms, we add and subtract $e^{\varphi} /\left[1+e^{\varphi}\right]$ from $\rho$ and then integrate by parts. After these steps the previous equation becomes

$$
\left\langle\rho-\frac{e^{\varphi}}{1+e^{\varphi}}, \varepsilon \varphi_{x x}+\varphi_{x}\left(1-\varepsilon \varphi_{x}\right)\left(\rho-\frac{1}{1+e^{\varphi}}\right)\right\rangle=0,
$$

where we used that $\sigma(a)=a(1-a)$ which implies that $\sigma(b)-\sigma(a)=(b-a)(1-a-b)$. To conclude the proof it is now enough to recall that, in view of item (ii), $\varphi$ solves (2.11).

Proof of Theorem 2.4.

(i) Recall that $\varepsilon_{0}\left(\varphi_{1}-\varphi_{0}\right)=1$. Let $\widetilde{\mathcal{F}}:=\left\{\varphi \in C^{1}([0,1]): \varphi(0)=\varphi_{0}, \varphi(1)=\right.$ $\left.\varphi_{1}, 0 \leq \varepsilon \varphi_{x} \leq 1\right\}$, and observe that $\tilde{\mathcal{F}} \subset \mathcal{F}$. Fix $\rho \in M$ and consider the integrodifferential operator $\mathcal{K}_{\rho}^{(1)}$ on $\widetilde{\mathcal{F}}$ defined by

$$
\left(\mathcal{K}_{\rho}^{(1)} \varphi\right)(x):=\varphi_{0}+\frac{x}{\varepsilon}-\left(\frac{1}{\varepsilon}-\frac{1}{\varepsilon_{0}}\right) \frac{\int_{0}^{x} \exp \left\{\int_{0}^{y} \mathcal{R}^{(1)}(\rho, \varphi ; z) d z\right\} d y}{\int_{0}^{1} \exp \left\{\int_{0}^{y} \mathcal{R}^{(1)}(\rho, \varphi ; z) d z\right\} d y},
$$


where

$$
\mathcal{R}^{(1)}(\rho, \varphi ; x):=\varepsilon^{-1}\left[\rho(x)-\frac{1}{1+e^{\varphi(x)}}\right] \varphi_{x}(x),
$$

which is informally obtained multiplying (2.11) by $\varphi_{x}$ and integrating the resulting equation. It is simple to check that if $\varepsilon_{0}-\varepsilon$ is small enough, then $\mathcal{K}_{\rho}^{(1)}: \widetilde{\mathcal{F}} \rightarrow \widetilde{\mathcal{F}}$.

Let $\varphi \in \mathcal{P}(\rho)$. By Proposition 4.1, $\varphi$ solves (2.11) a.e. and therefore it is also a fixed point of $\mathcal{K}_{\rho}^{(1)}$. Consider now the set $\widetilde{\mathcal{F}}$ endowed with the distance $\mathrm{d}(\varphi, \psi):=$ $\sup _{x}\left|\varphi_{x}(x)-\psi_{x}(x)\right|$. It is simple to show that, provided $\varepsilon_{0}-\varepsilon$ is small enough, the operator $\mathcal{K}_{\rho}^{(1)}$ is a contraction with respect to this distance. Namely, there exists $\alpha \in(0,1)$ such that $\mathrm{d}\left(\mathcal{K}_{\rho}^{(1)} \varphi, \mathcal{K}_{\rho}^{(1)} \psi\right) \leq \alpha \mathrm{d}(\varphi, \psi)$. This yields uniqueness of the fixed point of $\mathcal{K}_{\rho}^{(1)}$ and concludes the proof.

(ii) The proof is achieved by the same argument of the previous item by considering the integro-differential operator $\mathcal{K}_{\rho}^{(2)}$ on $\widetilde{\mathcal{F}}$ defined by

$$
\left(\mathcal{K}_{\rho}^{(2)} \varphi\right)(x):=\varphi_{0}+\left(\varphi_{1}-\varphi_{0}\right) \frac{\int_{0}^{x} \exp \left\{\int_{0}^{y} \mathcal{R}^{(2)}(\rho, \varphi ; z) d z\right\} d y}{\int_{0}^{1} \exp \left\{\int_{0}^{y} \mathcal{R}^{(2)}(\rho, \varphi ; z) d z\right\} d y}
$$

where

$$
\mathcal{R}^{(2)}(\rho, \varphi ; x):=\varepsilon^{-1}\left[\frac{1}{1+e^{\varphi(x)}}-\rho(x)\right]\left[1-\varepsilon \varphi_{x}(x)\right],
$$

which is informally obtained multiplying (2.11) by $1-\varepsilon \varphi_{x}$ and integrating the resulting equation.

(iii) Fix a strictly increasing function $\rho \in M \cap C^{1}([0,1])$ and let $\varphi \in \mathcal{P}(\rho)$. We shall show that the quadratic form given by the second variation of the functional $\mathcal{G}_{\varepsilon}(\rho, \cdot)$ evaluated at $\varphi$ is uniformly elliptic. This implies uniqueness of the critical point.

The second variation of $\mathcal{G}_{\varepsilon}(\rho, \cdot)$ evaluated at $\varphi$ is the quadratic form

$$
\left\langle h, \frac{\delta^{2}}{\delta \varphi^{2}} \mathcal{G}_{\varepsilon}(\rho, \varphi) h\right\rangle=\int_{0}^{1}\left[\frac{\varepsilon h_{x}^{2}}{\varphi_{x}\left(1-\varepsilon \varphi_{x}\right)}-\frac{e^{\varphi} h^{2}}{\left(1+e^{\varphi}\right)^{2}}\right] d x
$$

defined on functions $h$ in $\mathcal{H}_{0}^{1}$. Let $\psi:=\varphi_{x}$. Since $\rho$ and $\varphi$ are smooth, by differentiating 2.11) we deduce that

$$
\left(\frac{\varepsilon \psi_{x}}{\varphi_{x}\left(1-\varepsilon \varphi_{x}\right)}\right)_{x}+\frac{e^{\varphi} \psi}{\left(1+e^{\varphi}\right)^{2}}=-\rho_{x} .
$$

Performing the change of variables $h=\psi g$ in (4.3), which is legal because $\psi$ is smooth and strictly positive, a two lines computation based on the previous identity shows that

$$
\left\langle(\psi g), \frac{\delta^{2}}{\delta \varphi^{2}} \mathcal{G}_{\varepsilon}(\rho, \varphi)(\psi g)\right\rangle=\int_{0}^{1}\left[\frac{\varepsilon \psi^{2}}{\varphi_{x}\left[1-\varepsilon \varphi_{x}\right]} g_{x}^{2}+\psi \rho_{x} g^{2}\right] d x
$$

By item (ii) in Proposition 4.1 and the hypothesis $\rho_{x}>0$, we deduce that there exists a constant $c>0$ depending on $\rho$, but independent of $\varphi \in \mathcal{P}(\rho)$, such that

$$
\left\langle h, \frac{\delta^{2}}{\delta \varphi^{2}} \mathcal{G}_{\varepsilon}(\rho, \varphi) h\right\rangle \geq c\langle h, h\rangle
$$

which concludes the proof. 
Minimizers of $\mathcal{G}_{\varepsilon}(\rho, \cdot)$. We here analyze the minimizers of the functional $\mathcal{G}_{\varepsilon}(\rho, \cdot)$.

Lemma 4.2. Let $\rho \in M$ be smooth. Then any minimizer of $\mathcal{G}_{\varepsilon}(\rho, \cdot)$ is a fixed point of $K_{\rho}$, i.e.

$$
\mathcal{F}(\rho) \subset \mathcal{P}(\rho)
$$

Proof. To show that any minimizer of the variational problem (2.13) is a fixed point of $K_{\rho}$ we use a dynamical argument. Let $\varphi \in \mathcal{F}$ be such that $0 \leq \varepsilon \varphi_{x} \leq 1$ and consider the evolution equation

$$
\left\{\begin{array}{l}
v_{t}=\varepsilon v_{x x}+v_{x}\left(1-\varepsilon v_{x}\right)\left[\rho-\left(1+e^{v}\right)^{-1}\right] \\
v(t, 0)=\varphi_{0}, \quad v(t, 1)=\varphi_{1} \\
v(0, \cdot)=\varphi(\cdot)
\end{array}\right.
$$

Since $\rho$ is smooth, by classical results on uniformly parabolic equations, the solution $v$ is smooth in $(0, \infty) \times[0,1]$.

The functional $\mathcal{G}_{\varepsilon}(\rho, \cdot)$ is a Lyapunov functional for the evolution (4.4). Indeed, for $t>0$ we have

$$
\frac{d}{d t} \mathcal{G}_{\varepsilon}(\rho, v(t))=-\int_{0}^{1} v_{x}\left(1-\varepsilon v_{x}\right)\left[\frac{\varepsilon v_{x x}}{v_{x}\left(1-\varepsilon v_{x}\right)}+\rho-\frac{1}{1+e^{v}}\right]^{2} d x,
$$

which shows that $\mathcal{G}_{\varepsilon}(\rho, v(t)) \leq \mathcal{G}_{\varepsilon}(\rho, \varphi)$ provided $\varphi$ is smooth. By a standard approximation argument, we then extend this inequality to any $\varphi \in \mathcal{F}$.

Let $F=\rho-\left(1+e^{v}\right)^{-1}$ and $w=v_{x}$. Since $v_{t}(t, 0)=v_{t}(t, 1)=0, t>0$, a simple computation shows that $w$ solves

$$
\left\{\begin{array}{l}
w_{t}=\varepsilon w_{x x}+[w(1-\varepsilon w) F]_{x} \\
\varepsilon w_{x}(t, i)+w(t, i)[1-\varepsilon w(t, i)] F(t, i)=0, i=0,1, \\
w(0, \cdot)=\varphi_{x}(\cdot) .
\end{array}\right.
$$

Since $F_{x}$ is bounded on compact subsets of $(0, \infty) \times[0,1]$ and $\varphi_{x}$ is neither identically equal to 0 nor to $\varepsilon^{-1}$, Theorem 3.7 in $[33$ and the remark (ii) following it, imply that $0<\varepsilon w(t, x)<1$ for any $(t, x) \in(0, \infty) \times[0,1]$. This proves that $0<\varepsilon v_{x}<1$ in $(0, \infty) \times[0,1]$.

Let now $\varphi \in \mathcal{F}(\rho)$, i.e. $\varphi$ is a minimizer for $\mathcal{G}_{\varepsilon}(\rho, \cdot)$. We deduce $\mathcal{G}_{\varepsilon}(\rho, v(t))=$ $\mathcal{G}_{\varepsilon}(\rho, \varphi)$, namely that for each $t \geq 0$ the function $v(t)$ is a minimizer for $\mathcal{G}_{\varepsilon}(\rho, \cdot)$. Since, for $t>0$, the function $v(t)$ is smooth and $0<\varepsilon v_{x}(t)<1$, it satisfies the Euler-Lagrange equation (2.11). In particular, $v(t)$ is a fixed point of $K_{\rho}$, that is $v(t) \in \mathcal{P}(\rho)$. Since $v(t)$ converges to $\varphi$ strongly in $L^{1}([0,1])$ as $t \downarrow 0$, by taking the limit $t \downarrow 0$ in the equation $K_{\rho} v(t)=v(t)$, we conclude that $\varphi \in \mathcal{P}(\rho)$.

Since $\bar{\rho}_{\varepsilon}$ is smooth and strictly increasing, item (iii) in Theorem 2.4 implies that $\mathcal{P}\left(\bar{\rho}_{\varepsilon}\right)$ is a singleton. A simple computation shows that $s^{\prime}\left(\bar{\rho}_{\varepsilon}\right)$ solves the differential equation (2.11) for $\rho=\bar{\rho}_{\varepsilon}$. By the previous lemma, $s^{\prime}\left(\bar{\rho}_{\varepsilon}\right)$ is the unique minimizer of $\mathcal{G}_{\varepsilon}\left(\bar{\rho}_{\varepsilon}, \cdot\right)$. Hence,

$$
S_{\varepsilon}^{o}\left(\bar{\rho}_{\varepsilon}\right)=\inf _{\varphi \in \mathcal{F}} \mathcal{G}_{\varepsilon}\left(\bar{\rho}_{\varepsilon}, \varphi\right)=\mathcal{G}_{\varepsilon}\left(\bar{\rho}_{\varepsilon}, s^{\prime}\left(\bar{\rho}_{\varepsilon}\right)\right) .
$$

The previous lemma proves the first statement in Theorem 2.2 for smooth functions $\rho$. The proof of the general result is based in the following estimate.

Lemma 4.3. Fix $\varphi_{0}<\varphi_{1}$ and $\varepsilon \in\left(0, \varepsilon_{0}\right)$. There exists $\delta \in(0,1 / 2)$ such that for any $\rho \in M$ and any $\varphi \in \mathcal{F}(\rho)$ we have $\delta \leq \varepsilon \varphi_{x} \leq 1-\delta$ a.e. 
The most direct approach to prove this lemma would be by contradiction. Assuming the existence of a minimizer $\varphi$ with derivative not bounded away from 0 and $\varepsilon^{-1}$, we would need to construct a suitable function $\psi$ with derivative bounded away from 0 and $\varepsilon^{-1}$ such that $\mathcal{G}_{\varepsilon}(\rho, \psi)<\mathcal{G}_{\varepsilon}(\rho, \varphi)$. Our attempts in this direction have not however been successful and we shall prove Lemma 4.3 by an indirect route based on a geometric characterization of the minimizers, results from convex analysis, and Lemma 4.2 which implies the statement for smooth $\rho$. Postponing this proof, we show Theorem 2.2 .

Proof of Theorem 2.2: the inclusion $\mathcal{F}(\rho) \subset \mathcal{P}(\rho)$. Let $\rho \in M$ and $\varphi \in \mathcal{F}(\rho)$. In view of Lemma 4.3. we easily deduce that $\varphi$ satisfies the Euler-Lagrange equation (2.11) weakly, namely that for any $\lambda \in C_{0}^{\infty}([0,1])$

$$
\left\langle\varepsilon s^{\prime}\left(\varepsilon \varphi_{x}\right), \lambda_{x}\right\rangle+\left\langle-\rho+\frac{1}{1+e^{\varphi}}, \lambda\right\rangle=0 \text {. }
$$

We deduce there exists a constant $C$ such that for a.e. $x \in[0,1]$

$$
\varepsilon s^{\prime}\left(\varepsilon \varphi_{x}(x)\right)=C+\int_{0}^{x}\left[\frac{1}{1+e^{\varphi}}-\rho\right] d y .
$$

Recalling (2.12) and that $A=A(\varphi)$ is chosen so that $\left(K_{\rho} \varphi\right)(1)=\varphi_{1}$, it is straightforward to check that $K_{\rho} \varphi=\varphi$.

To conclude the proof of Theorem 2.2 it remains to prove the continuity properties of the functional $S_{\varepsilon}$. To this end, we consider the space $L^{1}([0,1])$ endowed with the weak topology and, recalling (2.9), we let $\Lambda: L^{1}([0,1]) \rightarrow(-\infty,+\infty]$ be the lower semicontinuous functional defined by

$$
\Lambda(\varphi):= \begin{cases}\int_{0}^{1}\left[s\left(\varepsilon \varphi_{x}\right)+\varphi-\log \left(1+e^{\varphi}\right)\right] d x & \text { if } \varphi \in \mathcal{F} \\ +\infty & \text { otherwise }\end{cases}
$$

where we understand that $\Lambda(\varphi)=+\infty$ unless $\varphi$ is absolutely continuous, and the density of the measure $d \varphi$, denoted by $\varphi_{x}$, satisfies $0 \leq \varepsilon \varphi_{x} \leq 1$ a.e. Note that the set $\{\varphi: \Lambda(\varphi)<+\infty\}$ is compact in $L^{1}([0,1])$. Recalling that we consider $L^{\infty}([0,1])$ endowed with the weak* topology, the Legendre transform of $\Lambda$ is defined as the function $\Lambda^{*}: L^{\infty}([0,1]) \rightarrow \mathbb{R}$ given by

$$
\Lambda^{*}(\rho):=\sup _{\varphi}\{\langle\rho, \varphi\rangle-\Lambda(\varphi)\}
$$

where the supremum is carried over all functions $\varphi$ in $L^{1}([0,1])$. Recalling (2.9), we also let $\mathcal{S}: L^{\infty}([0,1]) \rightarrow(-\infty,+\infty]$ be the functional defined by

$$
\mathcal{S}(\rho):=\int_{0}^{1} s(\rho) d x .
$$

In view of (2.13), the previous definitions imply

$$
S_{\varepsilon}^{o}=\mathcal{S}-\Lambda^{*}
$$

where we understand that $S_{\varepsilon}^{o}$, defined in (2.13), has been extended to a functional on $L^{\infty}([0,1])$ by setting $S_{\varepsilon}^{o}(\rho)=+\infty$ for $\rho \notin M$. 
Lemma 4.4. Fix $\varphi_{0}<\varphi_{1}, \varepsilon \in\left(0, \varepsilon_{0}\right)$ and a not empty closed subset $K$ of $\mathcal{F}$. Let $\Lambda_{K}^{*}: M \rightarrow \mathbb{R}$ be the functional defined by

$$
\Lambda_{K}^{*}(\rho):=\sup \{\langle\rho, \varphi\rangle-\Lambda(\varphi), \varphi \in K\} .
$$

Then $\Lambda_{K}^{*}$ is continuous.

Proof. The continuity of the map $M \ni \rho \mapsto\langle\rho, \varphi\rangle \in \mathbb{R}$ for a given $\varphi \in \mathcal{F}$ implies immediately the lower semicontinuity of $\Lambda_{K}^{*}$. To prove the upper semicontinuity, fix a sequence $\left\{\rho^{n}\right\} \subset M$ converging to $\rho$. Since $\Lambda_{K}^{*}\left(\rho^{n}\right)<\infty, n \geq 1$, there exists a sequence $\left\{\varphi^{n}\right\} \subset K$ such that

$$
\lim _{n \rightarrow \infty} \Lambda_{K}^{*}\left(\rho^{n}\right)=\lim _{n \rightarrow \infty}\left\{\left\langle\rho^{n}, \varphi^{n}\right\rangle-\Lambda\left(\varphi^{n}\right)\right\} .
$$

Since $\mathcal{F}$ is compact, by taking a subsequence, if necessary, we may assume that $\left\{\varphi^{n}\right\}$ converges in $\mathcal{F}$ to some $\varphi \in K$. As $\rho^{n} \rightarrow \rho$ in $M$ and $\varphi^{n} \rightarrow \varphi$ in $\mathcal{F}$ imply $\left\langle\rho^{n}, \varphi^{n}\right\rangle \rightarrow\langle\rho, \varphi\rangle$ and $\liminf _{n} \Lambda\left(\varphi^{n}\right) \geq \Lambda(\varphi)$, we deduce

$$
\limsup _{n} \Lambda_{K}^{*}\left(\rho^{n}\right) \leq\langle\rho, \varphi\rangle-\Lambda(\varphi) \leq \Lambda_{K}^{*}(\rho),
$$

which is the desired upper semicontinuity.

Proof of Theorem 2.2: conclusion. Recall (4.8) and observe that the convexity of $s$ immediately implies the lower semicontinuity of $\mathcal{S}$ on $M$. On the other hand, $\mathcal{S}: M \rightarrow \mathbb{R}$ is clearly continuous with respect to the strong topology of $L^{1}([0,1])$. In view of the decomposition (4.9), the proof of Theorem 2.2 is then concluded by applying Lemma 4.4 with $K=\mathcal{F}$.

In the sequel we shall need the following density result. Recall the definition of the set $M^{\circ}$ introduced in (4.1).

Lemma 4.5. Fix $\rho$ in $M$. There exist a function $\varphi$ in $\mathcal{F}(\rho)$, a sequence $\left\{\rho^{n}\right\} \subset M^{o}$, and a sequence $\left\{\varphi^{n}\right\} \subset \mathcal{F}, \varphi^{n} \in \mathcal{F}\left(\rho^{n}\right)$, such that $\rho^{n} \rightarrow \rho$ strongly in $L^{1}([0,1])$, $\varphi^{n} \rightarrow \varphi$ in the $C^{1}$ topology, and $\mathcal{G}_{\varepsilon}\left(\rho^{n}, \varphi^{n}\right) \rightarrow \mathcal{G}_{\varepsilon}(\rho, \varphi)$.

Proof. Fix $\rho$ in $M$ and pick a sequence $\left\{\rho^{n}\right\} \subset M^{o}$ converging to $\rho$ strongly in $L^{1}([0,1])$. Since $\mathcal{F}$ is compact and $\mathcal{G}_{\varepsilon}$ is lower semicontinuous, there exists a sequence $\left\{\varphi^{n}\right\} \subset \mathcal{F}$ such that $\varphi^{n} \in \mathcal{F}\left(\rho^{n}\right)$. By Lemma 4.2 we also have $\varphi^{n} \in \mathcal{P}\left(\rho^{n}\right)$. Hence, by item (ii) in Proposition 4.1 and Ascoli-Arzelà theorem, $\left\{\varphi^{n}\right\}$ is a precompact sequence in $C^{1}([0,1])$. In particular, by taking if necessary a subsequence, there exists a function $\varphi$ in $\mathcal{F} \cap C^{1}([0,1])$ such that $\varphi^{n} \rightarrow \varphi$ in $C^{1}([0,1])$. The last statement and the choice of $\left\{\rho^{n}\right\}$ imply $\mathcal{G}_{\varepsilon}\left(\rho^{n}, \varphi^{n}\right) \rightarrow \mathcal{G}_{\varepsilon}(\rho, \varphi)$.

It remains to show that $\varphi \in \mathcal{F}(\rho)$. By taking the limit $n \rightarrow \infty$ in the equation $K_{\rho^{n}} \varphi^{n}=\varphi^{n}$, we readily deduce that $\varphi \in \mathcal{P}(\rho)$. To show that $\varphi$ belongs to $\mathcal{F}(\rho)$ we proceed as follows. Since $\varphi^{n} \in \mathcal{F}\left(\rho^{n}\right)$, Lemma 4.4 and the continuity of $\mathcal{S}$ with respect to the strong $L^{1}([0,1])$ topology imply $\mathcal{G}_{\varepsilon}\left(\rho^{n}, \varphi^{n}\right)=S_{\varepsilon}^{o}\left(\rho^{n}\right) \rightarrow S_{\varepsilon}^{o}(\rho)$. Therefore, as $\mathcal{G}_{\varepsilon}\left(\rho^{n}, \varphi^{n}\right) \rightarrow \mathcal{G}_{\varepsilon}(\rho, \varphi)$, we deduce that $S_{\varepsilon}^{o}(\rho)=\mathcal{G}_{\varepsilon}(\rho, \varphi)$, i.e. $\varphi \in$ $\mathcal{F}(\rho)$.

Convexity considerations. Let $X$ and $X^{*}$ be vector spaces in duality with respect to $\langle\cdot, \cdot\rangle$. We consider $X$ and $X^{*}$ respectively endowed with the weak and weak* topology. Recall that $f: X \rightarrow(-\infty,+\infty]$ is Gâteaux differentiable at $x$ if there exists $\ell \in X^{*}$ such that

$$
\lim _{\lambda \downarrow 0} \frac{1}{\lambda}[f(x+\lambda v)-f(x)-\lambda\langle\ell, v\rangle]=0 \quad \text { for any } v \in X .
$$


In such a case we denote $\ell$ by $D_{\mathrm{G}} f(x)$. In general, we define the Gâteaux subdifferential $D_{\mathrm{G}}^{-} f(x)$ and Gâteaux super-differential $D_{\mathrm{G}}^{+} f(x)$ of $f$ at the point $x$ as the following, possibly empty, convex subsets of $X^{*}$

$$
\begin{aligned}
& D_{\mathrm{G}}^{+} f(x):=\left\{\ell \in X^{*}: \limsup _{\lambda \downarrow 0} \frac{1}{\lambda}[f(x+\lambda v)-f(x)-\lambda\langle\ell, v\rangle] \leq 0 \text { for any } v \in X\right\} \\
& D_{\mathrm{G}}^{-} f(x):=\left\{\ell \in X^{*}: \liminf _{\lambda \downarrow 0} \frac{1}{\lambda}[f(x+\lambda v)-f(x)-\lambda\langle\ell, v\rangle] \geq 0 \text { for any } v \in X\right\}
\end{aligned}
$$

in which we understand $D_{\mathrm{G}}^{ \pm} f(x)=\varnothing$ if $f(x)=+\infty$.

In the context of convex analysis, the sub-differential of a convex function $f$ : $X \rightarrow(-\infty,+\infty]$ at a point $x \in X$, here denoted by $\partial f(x)$, is the set of linear functionals $\ell \in X^{*}$ such that

$$
f(y)-f(x) \geq\langle\ell, y-x\rangle \quad \text { for any } y \in X
$$

in which we understand that $\partial f(x)=\varnothing$ if $f(x)=+\infty$. It is well-know, see e.g. [19, Prop. 1.5.3], that if $f$ is convex and Gâteaux differentiable at $x$ then $\partial f(x)=\left\{D_{\mathrm{G}} f(x)\right\}$. It is also simple to check that for any convex function $f: X \rightarrow$ $(-\infty,+\infty]$ the equality $D_{\mathrm{G}}^{-} f(x)=\partial f(x)$ holds for all $x \in X$.

Recall from (2.14) that the set $\mathcal{F}(\rho) \subset \mathcal{F}, \rho \in M$, represents the minimizers of $\mathcal{G}_{\varepsilon}(\rho, \cdot)$. In view of definition (4.6), the set $\mathcal{F}(\rho)$ coincides with the maximizers for the variational problem on the right hand side of (4.7). We here consider such variational problem for $\rho \in L^{\infty}([0,1])$ and still denote by $\mathcal{F}(\rho)$ the set of maximizers, i.e. $\mathcal{F}(\rho)=\arg \sup \left\{\langle\rho, \varphi\rangle-\Lambda(\varphi), \varphi \in L^{1}([0,1])\right\}$. For each $\rho \in L^{\infty}([0,1])$, the set $\mathcal{F}(\rho)$ is a not empty compact subset of $\mathcal{F}$. Given a set $A$ we $\operatorname{denote}$ by co $A$ its convex hull.

Proposition 4.6. The functional $\Lambda^{*}: L^{\infty}([0,1]) \rightarrow \mathbb{R}$ defined in (4.7) is lower semicontinuous and convex. Moreover, for each $\rho \in L^{\infty}([0,1])$ we have

$$
\begin{aligned}
& D_{\mathrm{G}}^{-} \Lambda^{*}(\rho)=\partial \Lambda^{*}(\rho)=\operatorname{co} \mathcal{F}(\rho), \\
& D_{\mathrm{G}}^{+} \Lambda^{*}(\rho)= \begin{cases}\{\varphi\} & \text { if } \mathcal{F}(\rho)=\{\varphi\} \text { for some } \varphi \in \mathcal{F}, \\
\varnothing & \text { otherwise. }\end{cases}
\end{aligned}
$$

In particular, $\Lambda^{*}$ is Gâteaux differentiable at $\rho$ if and only if $\mathcal{F}(\rho)$ is a singleton.

To prove this statement we need the following elementary result from convex analysis. We say that $f: X \rightarrow(-\infty,+\infty]$ has super-linear growth iff for each affine function on $X$, i.e. a map $X \ni x \mapsto\langle\ell, x\rangle+\alpha \in \mathbb{R}$ for some $\ell \in X^{*}$ and $\alpha \in \mathbb{R}$, there exists a compact $K=K_{\ell, \alpha} \subset X$ such that $f(x) \geq\langle\ell, x\rangle+\alpha$ for any $x \notin K$. Observe that if $f$ has super-linear growth, then $f$ is coercive.

Lemma 4.7. Let $f: X \rightarrow(-\infty,+\infty]$ be lower semicontinuous with super-linear growth. Denote by $f^{* *}$ the convex envelope of $f$, i.e. the largest convex and lower semicontinuous function below $f$. Then

$$
\text { co } \arg \inf \{f(x), x \in X\}=\arg \inf \left\{f^{* *}(x), x \in X\right\} .
$$

Proof. The inclusion co $\arg \inf \{f(x), x \in X\} \subset \arg \inf \left\{f^{* *}(x), x \in X\right\}$ is trivial; to prove the converse we shall argue by contradiction and assume, with no loss of generality, that inf $f=0$. Recall that the epigraph of $f$ is the subset of $X \times \mathbb{R}$ given by epi $f:=\{(x, t): f(x) \leq t\}$. The convex envelope $f^{* *}$ is then characterized by the identity co epi $f=$ epi $f^{* *}$, see e.g. [19, Proposition 1.3.2]. 
Assume, by contradiction, that there exists $\bar{x} \in \arg \inf \left\{f^{* *}(x), x \in X\right\}$ such that $\bar{x} \notin$ co $\arg \inf \{f(x), x \in X\}=: C$. Since $f$ is coercive and lower semicontinuous, its sub-level sets, and therefore $C$, are compact. By the Hahn-Banach theorem there exist $\alpha \in \mathbb{R}$ and $\ell \in X^{*}$ such that $C \subset\{x:\langle\ell, x\rangle>\alpha\}$ and $\bar{x} \in\{x:\langle\ell, x\rangle\langle\alpha\}$. Since $C$ is compact, we can find an open neighborhood $A$ of $C$ such that $A \subset\{x$ : $\langle\ell, x\rangle>\alpha\}$. Since $f$ has super-linear growth, we can also find a compact $K$ such that for any $x \notin K$ we have $f(x) \geq-\langle\ell, x\rangle+\alpha$. Let now

$$
m:=\inf \{f(x), x \notin A\}>0, \quad M:=\sup \{|\langle\ell, x\rangle|, x \in K\}<\infty,
$$

and set $\lambda:=\max \{1,(M+\alpha) / m\} \in[1, \infty)$. Consider the following half-spaces in $X \times \mathbb{R}$

$$
\Pi_{+}:=\{(x, t):\langle\ell, x\rangle+\lambda t \geq \alpha\} \quad \Pi_{-}:=\{(x, t):\langle\ell, x\rangle+\lambda t \leq \alpha\}
$$

and observe that $(\bar{x}, 0)$ belongs to the interior of $\Pi_{-}$. It is also easy to check that epi $f \subset \Pi_{+}$. Therefore, as co epi $f$ is the intersection of all the half-spaces containing epi $f$, we deduce that $(\bar{x}, 0) \notin$ co epi $f$ which yields the desired contradiction.

Proof of Proposition 4.6. The lower semicontinuity and convexity of $\Lambda^{*}$ follows trivially from its definition. Given $\rho \in L^{\infty}([0,1]$, set

$$
\widetilde{\mathcal{F}}(\rho):=\arg \sup \left\{\langle\rho, \varphi\rangle-\Lambda^{* *}(\varphi), \varphi \in L^{1}([0,1])\right\}
$$

where, we recall, $\Lambda^{* *}$ denotes the convex envelope of $\Lambda$. As $\left\{\varphi \in L^{1}([0,1]): \Lambda(\varphi)<\right.$ $\infty\}$ is compact, $\Lambda$ has super-linear growth and Lemma 4.7 yields $\widetilde{\mathcal{F}}(\rho)=\operatorname{co} \mathcal{F}(\rho)$. On the other hand, by e.g. [19, Prop. 1.4.1 and Cor. 1.4.1,],

$$
\Lambda^{*}(\rho)=\Lambda^{* * *}(\rho):=\sup _{\varphi}\left\{\langle\rho, \varphi\rangle-\Lambda^{* *}(\varphi)\right\} .
$$

Since we are now dealing with Legendre duality between convex functions, by e.g. 19. Prop. 1.5.1 and Cor. 1.5.2], $\varphi \in \partial \Lambda^{*}(\rho)$ iff $\rho \in \partial \Lambda^{* *}(\varphi)$ iff $\varphi \in \widetilde{\mathcal{F}}(\rho)$. This concludes the proof of the equality $\partial \Lambda^{*}(\rho)=\operatorname{co} \mathcal{F}(\rho)$.

We claim that if $\mathcal{F}(\rho)=\{\varphi\}$ for some $\varphi \in \mathcal{F}$ then $\varphi \in D_{\mathrm{G}}^{+} \Lambda^{*}(\rho)$. This statement completes the proof of the proposition. Indeed, if $D_{\mathrm{G}}^{+} \Lambda^{*}(\rho) \neq \varnothing$ and $D_{\mathrm{G}}^{-} \Lambda^{*}(\rho) \neq \varnothing$ then $\Lambda^{*}(\rho)$ is necessary Gâteaux differentiable at $\rho$.

To prove the claim, given $v \in L^{\infty}([0,1])$ and $\lambda>0$, pick $\varphi_{\lambda} \in \mathcal{F}(\rho+\lambda v)$. By the very definition of $\Lambda^{*}(\rho)$,

$$
\Lambda^{*}(\rho+\lambda v)-\Lambda^{*}(\rho)-\lambda\langle\varphi, v\rangle \leq \lambda\left\langle\varphi_{\lambda}-\varphi, v\right\rangle .
$$

The proof will therefore be completed once we show that any element in $\mathcal{F}(\rho+\lambda v)$ converges, as $\lambda \downarrow 0$, weakly in $L^{1}([0,1])$ to $\varphi$. Since we assumed $\mathcal{F}(\rho)$ to be a singleton, this is a straightforward consequence of the lower semicontinuity of $\Lambda$ and the compactness of $\mathcal{F}$.

The proof of Lemma4.3 is basically achieved by Lemma4.2. Proposition4.6 and the following general result in convex analysis, which is proven in [29. Let $B$ be a separable Banach space and $f: B \rightarrow \mathbb{R}$ a continuous convex function. Given $x \in B$ and $\ell \in \partial f(x)$ we say that $\ell$ is approximable by unique tangent functionals iff there exists a sequence $\left\{x_{n}\right\} \subset B$ converging (in norm) to $x$ such that $\partial f\left(x_{n}\right)=\left\{\ell_{n}\right\}$ for some $\ell_{n} \in B^{*}$ and $\ell_{n} \rightarrow \ell$ in the weak* topology of $B^{*}$. The collection of elements in $\partial f(x)$ approximable by unique tangent functionals is denoted by $\partial_{\text {app }} f(x)$. 
Theorem 4.8. Let $B$ be a separable Banach space and $f: B \rightarrow \mathbb{R}$ be a continuous convex function. Then for each $x \in B$

$$
\partial f(x)=\overline{\mathrm{co}} \partial_{\mathrm{app}} f(x),
$$

where $\overline{\mathrm{co}}$ denotes the weak ${ }^{*}$ closure of the convex hull.

As the previous theorem requires to work in a separable Banach space, we extend the functional $\Lambda^{*}: L^{\infty}([0,1]) \rightarrow \mathbb{R}$, as defined in (4.7), to a functional on the space $L^{1}([0,1])$ endowed with the strong topology. To avoid ambiguities we shall denote the extended functional by $\widehat{\Lambda}$. In other words, we let $\widehat{\Lambda}:\left(L^{1}([0,1])\right.$, strong $) \rightarrow \mathbb{R}$ be the functional defined by

$$
\widehat{\Lambda}(\rho):=\sup _{\varphi}\{\langle\rho, \varphi\rangle-\Lambda(\varphi)\}
$$

where the supremum is carried over all $\varphi$ in $L^{1}([0,1])$ such that $\Lambda(\varphi)<\infty$.

Clearly, $\widehat{\Lambda}$ is convex and, by the argument used in Lemma 4.4, continuous with respect to the strong topology of $L^{1}\left([0,1]\right.$. We claim that for each $\rho \in L^{\infty}([0,1])$ we have

$$
\partial \widehat{\Lambda}(\rho)=\partial \Lambda^{*}(\rho)
$$

By the previous identity we mean that any element in $\partial \Lambda^{*}(\rho)$, which a priori belongs only to $L^{1}([0,1])$, belongs also to $L^{\infty}([0,1])$ and it is an element in $\partial \widehat{\Lambda}(\rho)$. Indeed, on the one hand, it follows from the definition of sub-differentials that $\partial \widehat{\Lambda}(\rho) \subset \partial \Lambda^{*}(\rho)$ for each $\rho \in L^{\infty}([0,1])$. The reverse inclusion follows from the definition of subdifferentials, the fact, proven in Proposition 4.6. that $\partial \Lambda^{*}(\rho)=\operatorname{co} \mathcal{F}(\rho)$ and the continuity of $\widehat{\Lambda}$ with respect to the strong topology of $L^{1}([0,1])$.

Proof of Lemma 4.3. We shall consider the integral operator $K_{\rho}: \mathcal{F} \rightarrow \mathcal{F}$, as defined in (2.12), for $\rho \in L^{1}([0,1])$ instead of $L^{\infty}([0,1])$. To avoid ambiguities, denote by $\widehat{\mathcal{P}}(\rho)$ the set of $\varphi \in \mathcal{F}$ which are fixed points of $K_{\rho}, \rho \in L^{1}([0,1])$. By the proof of Proposition 4.1, $\widehat{\mathcal{P}}(\rho)$ is not empty and any $\varphi \in \widehat{\mathcal{P}}(\rho)$ belongs to $C^{1}([0,1])$. Furthermore, there exists $\delta>0$ depending on $\varphi_{0}<\varphi_{1}, \varepsilon \in\left(0, \varepsilon_{0}\right)$, and $|\rho|_{L^{1}}$ such that any $\varphi \in \widehat{\mathcal{P}}(\rho)$ satisfies the bound $\delta \leq \varepsilon \varphi_{x} \leq 1-\delta$. We stress that $\delta$ depends on $\rho$ only via $|\rho|_{L^{1}}$.

We claim that if $\rho \in L^{1}([0,1])$ is such that $\partial \widehat{\Lambda}(\rho)=\{\varphi\}$ for some $\varphi \in L^{\infty}([0,1])$, then $\varphi \in \widehat{\mathcal{P}}(\rho)$. Postponing the proof of this claim, we first conclude the proof of the lemma. Fix $\rho \in L^{\infty}([0,1])$ and $\varphi \in \partial_{\text {app }} \widehat{\Lambda}(\rho)$. By definition, there exists a sequence $\left\{\rho^{n}\right\} \subset L^{1}([0,1])$ converging to $\rho$ strongly in $L^{1}([0,1])$ such that $\partial \widehat{\Lambda}\left(\rho^{n}\right)=\left\{\varphi^{n}\right\}$ and $\varphi^{n} \rightarrow \varphi$ weak $^{*}$ in $L^{\infty}([0,1])$. In view of the previous claim $\varphi^{n} \in C^{1}([0,1])$ and there exists $\delta_{n}>0$, depending only on $\left|\rho^{n}\right|_{L^{1}}$, such that $\delta_{n} \leq \varepsilon \varphi_{x}^{n} \leq 1-\delta_{n}$. Since $\rho^{n}$ converges to $\rho$ in $L^{1}([0,1]), \delta=\min \left\{\delta_{n}: n \geq 1\right\}>0$. By duality, it is readily shown that the map $\psi \mapsto\left|\psi_{x}\right|_{L^{\infty}}$ is lower semicontinuous with respect to the weak* convergence in $L^{\infty}([0,1])$. Therefore $\left|\varepsilon \varphi_{x}\right|_{L^{\infty}} \leq 1-\delta$ and, by the same argument, $\left|1-\varepsilon \varphi_{x}\right|_{L^{\infty}} \leq 1-\delta$, that is, $\delta \leq \varepsilon \varphi_{x} \leq 1-\delta$ a.e.

Lemma 4.3 thus holds for $\varphi$ in $\partial_{\text {app }} \widehat{\Lambda}(\rho)$. Fix now $\rho \in L^{\infty}([0,1]),|\rho|_{L^{\infty}} \leq 1$, and $\varphi \in \mathcal{F}(\rho)$. By Proposition 4.6 and by (4.12), $\varphi$ belongs to $\partial \Lambda^{*}(\rho)=\partial \widehat{\Lambda}(\rho)$. Hence, by Theorem 4.8 and by the first part of the proof, there exists a sequence $\left\{\psi^{n}\right\}, \psi^{n}$ convex combinations of elements in $\partial_{\text {app }} \widehat{\Lambda}(\rho)$, such that $\delta \leq \varepsilon \psi_{x}^{n} \leq 1-\delta$ a.e. for some $\delta>0$ and $\psi^{n}$ converges in the weak* topology of $L^{\infty}([0,1])$ to $\varphi$. To 
conclude, it remains to recall the lower semicontinuity of $\psi \mapsto\left|\psi_{x}\right|_{L^{\infty}}$ with respect to the weak* convergence in $L^{\infty}([0,1])$.

We turn now to the proof of the claim. Pick a sequence of smooth functions $\left\{\rho^{n}\right\}$ converging to $\rho$ strongly in $L^{1}([0,1])$ and choose $\varphi^{n} \in \mathcal{F}\left(\rho^{n}\right)$. By Proposition 4.6 and (4.12), $\varphi^{n} \in \partial \Lambda^{*}\left(\rho^{n}\right)=\partial \widehat{\Lambda}\left(\rho^{n}\right)$. On the other hand, by Lemma 4.2, as $\rho^{n}$ is smooth, $\varphi^{n} \in \widehat{\mathcal{P}}\left(\rho^{n}\right)=\mathcal{P}\left(\rho^{n}\right)$. In particular $\left\{\varphi^{n}\right\} \subset \mathcal{F}$. By taking, if necessary, a subsequence, the compactness of $\mathcal{F}$ now yields the existence of $\psi \in \mathcal{F}$ such that $\varphi^{n} \rightarrow \psi$ in $\mathcal{F}$. We can thus take the limit $n \rightarrow \infty$ in the equation $\varphi^{n}=K_{\rho^{n}} \varphi^{n}$ and conclude that $\psi \in \widehat{\mathcal{P}}(\rho)$. Finally, by using the definition of sub-differentials, we easily deduce that $\psi \in \partial \widehat{\Lambda}(\rho)$. Since we assumed $\partial \widehat{\Lambda}(\rho)=\{\varphi\}$, we conclude $\psi=\varphi$.

\section{THE QUASI-POTENTIAL}

Relying on the properties of the static variational problem presented in the previous section, we prove here the main result namely, the identity between the quasi potential $V_{\varepsilon}$ and the functional $S_{\varepsilon}$. The proof is organized as follows. We first prove the equality $\widehat{V}_{\varepsilon}=V_{\varepsilon}$. We then show that the algorithm presented below the statement of Theorem 2.3 provides a legal test path for the variational problem (2.7). By elementary computations, basically the ones described in the context of Hamiltonian formalism, we deduce the inequality $\widehat{V}_{\varepsilon} \leq S_{\varepsilon}$. Finally, by using the variational definition (2.3) of the action functional, we prove the inequality $V_{\varepsilon} \geq S_{\varepsilon}$ by exhibiting a suitable test function $H$. In these arguments, all the computations will be performed for smooth paths and we will use density results to extend the bounds to arbitrary paths.

The identity $\widehat{V}_{\varepsilon}=V_{\varepsilon}$. We prove in this subsection that the variational problems (2.4) and (2.7) are equivalent. We emphasize that this statement does not depend on the specific form of the flux $f$ and the mobility $\sigma$. The argument relies on two results. The first one asserts that if the action of a path $u$ in $\mathcal{U}\left(\bar{\rho}_{\varepsilon}\right)$ is finite, then there exists a sequence $t_{n} \rightarrow-\infty$ such that $u\left(t_{n}\right)-\bar{\rho}_{\varepsilon}$ converges to 0 in $\mathcal{H}_{0}^{1}$, recall the notation for the Sobolev spaces introduced before the statement of Theorem 2.1. The second one asserts that if a function $\rho \in M$ is such that $\rho-\bar{\rho}_{\varepsilon}$ is small in $\mathcal{H}_{0}^{1}$, then there exists a path in a time interval of length one which connects $\bar{\rho}_{\varepsilon}$ to $\rho$ and has small action. In this subsection we drop the subscript $\varepsilon$ also in the stationary solution $\bar{\rho}_{\varepsilon}$ and denote its derivative by $\bar{\rho}_{x}$.

Lemma 5.1. Fix $T>0$ and a path $u$ in $C([-T, 0], M)$ such that $I_{[-T, 0]}(u)<\infty$. Then,

$$
\begin{aligned}
& \frac{\varepsilon}{8} \int_{-T}^{0}|u(t)-\bar{\rho}|_{\mathcal{H}_{0}^{1}}^{2} d t+\frac{1}{4}|u(0)-\bar{\rho}|_{L^{2}}^{2} \\
& \quad \leq \frac{1}{4}|u(-T)-\bar{\rho}|_{L^{2}}^{2}+I_{[-T, 0]}(u)+\frac{1}{2 \varepsilon} \int_{-T}^{0}|u(t)-\bar{\rho}|_{L^{2}}^{2} d t .
\end{aligned}
$$

Proof. The proof of this lemma is similar to the one of Lemma 4.9 in [10] or the one of Lemma 4.2 in 22. We thus just sketch the argument.

Fix $T>0$ and recall the definition of the linear functional $L_{u}$ introduced in (2.2). By definition of the action functional $I_{[-T, 0]}$, for any function $H$ in $C_{0}^{\infty}([-T, 0] \times$ 
$[0,1])$

$$
L_{u}(H)-\varepsilon\left\langle\left\langle H_{x}, \sigma(u) H_{x}\right\rangle\right\rangle \leq I_{[-T, 0]}(u) .
$$

Take $H=(1 / 2)(u-\bar{\rho})$. This function is not smooth and does not satisfy the boundary conditions at $-T$ and 0 , but can be approximated by such smooth functions in the norms needed for our purposes. This is presented with all details in the proofs of Lemma 4.9 in [10] and Lemma 4.2 in [22]. For instance, to match the boundary conditions at $-T$ and 0 , we multiply $H$ by a time dependent function which vanishes at $-T$ and 0 and which is close to the indicator of the interval $[0, T]$.

Integrating by parts and adding to the previous expression $\left\langle\left\langle f(\bar{\rho})_{x}-\varepsilon \bar{\rho}_{x x}, H\right\rangle\right\rangle$, which vanishes, the previous equation implies

$$
\begin{gathered}
\frac{1}{4}|u(0)-\bar{\rho}|_{L^{2}}^{2}+\frac{\varepsilon}{2}\left\langle\left\langle u_{x}-\bar{\rho}_{x}, u_{x}-\bar{\rho}_{x}\right\rangle\right\rangle-\frac{\varepsilon}{4}\left\langle\left\langle u_{x}-\bar{\rho}_{x}, \sigma(u)\left[u_{x}-\bar{\rho}_{x}\right]\right\rangle\right\rangle \\
\leq I_{[-T, 0]}(u)+\frac{1}{4}|u(-T)-\bar{\rho}|_{L^{2}}^{2}+\frac{1}{2}\left\langle\left\langle f(u)-f(\bar{\rho}), u_{x}-\bar{\rho}_{x}\right\rangle\right\rangle
\end{gathered}
$$

Since $\sigma(u) \leq 1 / 4$, it remains to apply Schwarz inequality to bound the last term and to recall that $f$ is Lipschitz with Lipschitz constant one.

Lemma 5.2. Fix a path $u$ in $\mathcal{U}(\bar{\rho})$ such that $I(u)<\infty$. Then

$$
\lim _{t \rightarrow-\infty}|u(t)-\bar{\rho}|_{L^{2}}=0 \quad \text { and } \quad \lim _{n \rightarrow \infty} \int_{-(n+1)}^{-n}|u(t)-\bar{\rho}|_{\mathcal{H}_{0}^{1}}^{2} d t=0 .
$$

Proof. We first show that

$$
\lim _{n \rightarrow \infty} \int_{-(n+1)}^{-n}\langle u(t)-\bar{\rho}, u(t)-\bar{\rho}\rangle d t=0 .
$$

Let $\left\{e_{k}, k \geq 1\right\}$ be the complete orthonormal system of $L_{2}([0,1])$ given by $e_{k}(x)=\sqrt{2} \sin (k \pi x)$ and set $\theta_{k}(t)=\left\langle u(t)-\bar{\rho}, e_{k}\right\rangle, k \geq 1$. The integral in the previous formula can be written as

$$
\sum_{k \geq 1} \int_{-(n+1)}^{-n} \theta_{k}(t)^{2} d t \leq \sum_{k=1}^{k_{0}} \int_{-(n+1)}^{-n} \theta_{k}(t)^{2} d t+\frac{1}{k_{0}^{2}} \sum_{k \geq 1} \int_{-(n+1)}^{-n} k^{2} \theta_{k}(t)^{2} d t
$$

for any $k_{0} \geq 1$. Since $e_{k}$ is an eigenfunction of the Laplacian with Dirichlet boundary conditions, the second term is equal to the time integral of $\left(\pi k_{0}\right)^{-2}|u(t)-\bar{\rho}|_{\mathcal{H}_{0}^{1}}^{2}$. Therefore, by Lemma 5.1, the last expression is less than or equal to

$$
\begin{aligned}
& \sum_{k=1}^{k_{0}} \int_{-(n+1)}^{-n}\left\langle u(t)-\bar{\rho}, e_{k}\right\rangle^{2} d t+\frac{2}{\pi^{2} k_{0}^{2} \varepsilon}|u(-(n+1))-\bar{\rho}|_{L^{2}}^{2} \\
& \quad+\frac{1}{\pi^{2} k_{0}^{2}}\left\{\frac{8}{\varepsilon} I_{[-(n+1),-n]}(u)+\frac{4}{\varepsilon^{2}} \int_{-(n+1)}^{-n}|u(t)-\bar{\rho}|_{L^{2}}^{2} d t\right\} .
\end{aligned}
$$

Since $u(t)-\bar{\rho}$ is absolutely bounded by 1 and since $I_{[-(n+1),-n]}(u) \leq I(u)$, the previous expression is bounded by

$$
\sum_{k=1}^{k_{0}} \int_{-(n+1)}^{-n}\left\langle u(t)-\bar{\rho}, e_{k}\right\rangle^{2} d t+\frac{1}{\pi^{2} k_{0}^{2}}\left\{\frac{2}{\varepsilon}+\frac{4}{\varepsilon^{2}}+\frac{8}{\varepsilon} I(u)\right\} .
$$

Since $u(t)$ converges weakly in $L^{2}([0,1])$ to $\bar{\rho}$, as $t \rightarrow-\infty$, to conclude the proof of the claim (5.1) it remains to let $n \rightarrow \infty$ and then $k_{0} \rightarrow \infty$. 
Since $u$ belongs to $C((-\infty, 0], M)$, the function $t \mapsto|u(t)-\bar{\rho}|_{L^{2}}$ is lower semicontinuous. In particular, there exists $s_{n} \in[-n,-(n-1)]$ such that

$$
\left|u\left(s_{n}\right)-\bar{\rho}\right|_{L^{2}}^{2}=\min _{-n \leq t \leq-(n-1)}|u(t)-\bar{\rho}|_{L^{2}}^{2} .
$$

By applying Lemma 5.1 in the time interval $\left[s_{n+1}, t\right]$ with $t \in[-n,-(n-1)]$ we deduce

$$
\sup _{-n \leq t \leq-(n-1)}|u(t)-\bar{\rho}|_{L^{2}}^{2} \leq 4 I_{[-(n+1),-(n-1)]}(u)+\left(1+\frac{2}{\varepsilon}\right) \int_{-(n+1)}^{-(n-1)}|u(t)-\bar{\rho}|_{L^{2}}^{2} d t,
$$

where we bounded $\left|u\left(s_{n+1}\right)-\bar{\rho}\right|_{L^{2}}^{2}$ by the time integral of $|u(t)-\bar{\rho}|_{L^{2}}^{2}$ over the interval $[-(n+1),-n]$. In view of the hypothesis $I(u)<\infty$ and (5.1), the first statement of the lemma follows from the previous estimate. The second one is a direct consequence of the first and Lemma 5.1

Fix a path $u$ in $\mathcal{U}\left(\bar{\rho}_{\varepsilon}\right)$ such that $I(u)<\infty$. It follows from the previous lemma that there exists a sequence $t_{n} \rightarrow-\infty$ such that

$$
\lim _{n}\left|u\left(t_{n}\right)-\bar{\rho}\right|_{\mathcal{H}_{0}^{1}}=0 \text {. }
$$

Lemma 5.3. Suppose that $\rho$ is a function in $M$ such that

$$
|\rho-\bar{\rho}|_{\mathcal{H}_{0}^{1}} \leq \frac{1}{2} \min \left\{\rho_{0}, 1-\rho_{1}\right\} .
$$

Then, there exists a constant $C_{0}$, depending only on $\rho_{0}$ and $\rho_{1}$, such that for any $T>0$

$$
\inf \left\{I_{[-T, 0]}(u), u(-T)=\bar{\rho}, u(0)=\rho\right\} \leq C_{0}\left(T+\frac{1}{T}\right)\left(\varepsilon+\frac{1}{\varepsilon}\right)|\rho-\bar{\rho}|_{\mathcal{H}_{0}^{1}}^{2} .
$$

Proof. We have to exhibit a path whose action can be estimated by the right hand side of the inequality appearing in the statement of the lemma. We claim that the straight one $u(t)=[1+(t / T)] \rho-(t / T) \bar{\rho}, t \in[-T, 0]$, fulfills the requirements.

Since $\rho_{0} \leq \bar{\rho}(x) \leq \rho_{1}$, it follows from the assumption of the lemma and from the elementary estimate between the $L^{\infty}$ norm and the $\mathcal{H}_{0}^{1}$ norm, namely, from the estimate $|h|_{L^{\infty}}^{2} \leq \int_{0}^{1} h_{x}^{2} d x$, that $\rho_{0} / 2 \leq u \leq \rho_{1}+\left(1-\rho_{1}\right) / 2$. In particular, $\sigma(u) \geq c_{0}>0$ for some constant $c_{0}$ which depends only on $\rho_{0}$ and $\rho_{1}$.

Recall the definition (2.3) of the action functional $I_{[-T, 0]}$. Fix a function $H$ in $C_{0}^{\infty}([-T, 0] \times[0,1])$, we need to estimate three terms to get a bound on $I_{[-T, 0]}(u)$. The first one is $\left\langle\left\langle u_{t}, H\right\rangle\right\rangle$. Since $u_{t}=(\rho-\bar{\rho}) / T$, by using Schwarz inequality and $\langle h, h\rangle \leq\left\langle h_{x}, h_{x}\right\rangle$, we deduce

$$
\left\langle\left\langle u_{t}, H\right\rangle \leq \frac{\varepsilon c_{0}}{2}\left\langle\left\langle H_{x}, H_{x}\right\rangle\right\rangle+\frac{1}{2 \varepsilon c_{0} T}|\rho-\bar{\rho}|_{\mathcal{H}_{0}^{1}}^{2} .\right.
$$

The second and third terms are estimated as follows. Since $f(\bar{\rho})_{x}+\varepsilon \bar{\rho}_{x x}=0$,

$$
\left\langle\left\langle f(u)_{x}-\varepsilon u_{x x}, H\right\rangle\right\rangle=-\left\langle\left\langle f(u)-f(\bar{\rho}), H_{x}\right\rangle\right\rangle+\varepsilon\left\langle\left\langle u_{x}-\bar{\rho}_{x}, H_{x}\right\rangle\right\rangle .
$$

Since $u-\bar{\rho}=[1+(t / T)][\rho-\bar{\rho}]$, again by Schwarz inequality

$$
\left\langle\left\langle f(u)_{x}-\varepsilon u_{x x}, H\right\rangle \leq \frac{\varepsilon c_{0}}{2}\left\langle\left\langle H_{x}, H_{x}\right\rangle\right\rangle+\frac{T}{c_{0}}\left(\varepsilon+\frac{1}{\varepsilon}\right)|\rho-\bar{\rho}|_{\mathcal{H}_{0}^{1}}^{2},\right.
$$

where we used the fact that $f^{\prime}$ is absolutely bounded by 1 and again the estimate $\langle h, h\rangle \leq\left\langle h_{x}, h_{x}\right\rangle$. Since $\sigma(u) \geq c_{0}$, the lemma follows from the previous bounds and (2.3). 
We are now ready to prove the equivalence between the variational problems (2.4) and (2.7).

Proof of Theorem [2.3: the identity $\widehat{V}_{\varepsilon}=V_{\varepsilon}$. Any path $u$ in $C([-T, 0] ; M)$ such that $u(-T)=\bar{\rho}$ may be extended to a path in $\mathcal{U}(\bar{\rho})$ by setting $u(t)=\bar{\rho}$, for $t \in$ $(-\infty,-T)$. The inequality $\widehat{V}_{\varepsilon} \leq V_{\varepsilon}$ follows trivially.

To prove the reverse inequality, fix $\rho$ in $M$ such that $\widehat{V}_{\varepsilon}(\rho)<\infty$. Fix $0<\delta \leq$ $(1 / 2) \min \left\{\rho_{0}, 1-\rho_{1}\right\}$ and let $u^{\delta} \in \mathcal{U}(\bar{\rho})$ be such that $u^{\delta}(0)=\rho$ and $I\left(u^{\delta}\right) \leq \widehat{V}_{\varepsilon}(\rho)+\delta$. By (5.2), there exists $T>0$ such that

$$
\left|u^{\delta}(-T)-\bar{\rho}\right|_{\mathcal{H}_{0}^{1}} \leq \delta .
$$

By Lemma 5.3, there exists a path $v$ in $C([-1,0], M)$ such that $v(-1)=\bar{\rho}, v(0)=$ $u^{\delta}(-T)$, and $I_{[-1,0]}(v) \leq 2 C_{0}\left(\varepsilon+\varepsilon^{-1}\right) \delta^{2}$. Consider the path $w$ in $C([-T-1,0], M)$ defined by $w(t)=v(t+T), t \in[-(T+1),-T], w(t)=u^{\delta}(t), t \in(-T, 0]$. Clearly, $w(-T-1)=\bar{\rho}, w(0)=\rho$ and

$$
I_{[-(T+1), 0]}(w)=I_{[-1,0]}(v)+I_{[-T, 0]}\left(u^{\delta}\right) \leq \widehat{V}_{\varepsilon}(\rho)+\delta+2 C_{0}\left(\varepsilon+\frac{1}{\varepsilon}\right) \delta^{2},
$$

which, by the arbitrariness of $\delta$, yields $V_{\varepsilon}(\rho) \leq \widehat{V}_{\varepsilon}(\rho)$ and concludes the proof.

Upper bound for quasi-potential. Recalling (4.1) and (3.8), the first two lemmata below state that if $\rho$ belongs to $M^{o}$ and $(\varphi, \rho) \in \Sigma$ then we can construct a solution to the canonical equations (3.7) converging to $\left(s^{\prime}\left(\bar{\rho}_{\varepsilon}\right), \bar{\rho}_{\varepsilon}\right)$ as $t \rightarrow-\infty$.

Fix a continuous function $\varphi:[0,1] \rightarrow\left[\varphi_{0}, \varphi_{1}\right]$ satisfying the boundary conditions $\varphi(0)=\varphi_{0}, \varphi(1)=\varphi_{1}$, and consider the parabolic equation

$$
\left\{\begin{array}{l}
\psi_{t}=\varepsilon \psi_{x x}+\frac{e^{\psi}-1}{e^{\psi}+1} \psi_{x}\left(1-\varepsilon \psi_{x}\right), \\
\psi(t, 0)=\varphi_{0}, \quad \psi(t, 1)=\varphi_{1}, \\
\psi(0, \cdot)=\varphi(\cdot) .
\end{array}\right.
$$

A classical solution $\psi$ of $(5.3)$ is a function $\psi:[0, \infty) \times[0,1] \rightarrow \mathbb{R}$ such that $\psi$ is continuous in $[0, \infty) \times[0,1], \psi_{t}, \psi_{x}$ and $\psi_{x x}$ are continuous in $(0, \infty) \times(0,1)$, and $\psi$ satisfies the identities (5.3). Let $F:[0, \infty) \times[0,1] \rightarrow[0,1]$ be a classical solution to the viscous Burgers equation (2.1). A simple computation shows that $\psi=s^{\prime}(F)=\log [F /(1-F)]$ is a classical solution to (5.3).

Lemma 5.4. Fix an absolutely continuous function $\varphi:[0,1] \rightarrow\left[\varphi_{0}, \varphi_{1}\right]$ satisfying the boundary conditions $\varphi(0)=\varphi_{0}, \varphi(1)=\varphi_{1}$ and $0 \leq \varepsilon \varphi_{x} \leq 1$. There exists a unique classical solution $\psi$ to (5.3). Moreover, this solution satisfies $0<\varepsilon \psi_{x}<1$ for $(t, x) \in(0, \infty) \times[0,1]$. Finally, as $t \rightarrow+\infty$ the function $\psi(t)$ converges to $s^{\prime}\left(\bar{\rho}_{\varepsilon}\right)=\log \left[\bar{\rho}_{\varepsilon} /\left(1-\bar{\rho}_{\varepsilon}\right)\right]$ in the $C^{3}([0,1])$ topology uniformly in $\varphi$.

Proof. We start with existence. Set $F_{0}:=e^{\varphi} /\left[1+e^{\varphi}\right]$. By assumption, the function $F_{0}$ is continuous, bounded below by $\rho_{0}$, bounded above by $\rho_{1}$ and satisfies $F_{0}(0)=$ $\rho_{0}, F_{0}(1)=\rho_{1}$. By Theorem 4.4 in Chapter 6 of [28], there exists a unique classical solution, denoted by $F=F(t, x)$, to the viscous Burgers (2.1) with initial condition $F_{0}$. By maximum principle, $\rho_{0} \leq F \leq \rho_{1}$. The function $\psi:[0, \infty) \times[0,1] \rightarrow \mathbb{R}$ given by $\psi:=s^{\prime}(F)=\log [F /(1-F)]$ is therefore well defined. An elementary computation that we omit, relying on the special form of the flux given by $f(u)=$ $u(1-u)$, shows that $\psi$ is a classical solution to (5.3). Uniqueness follows from a 
similar argument. Indeed, if $\psi$ is a classical solution to (5.3), then $F=e^{\psi} /\left[1+e^{\psi}\right]$ is a classical solution to (2.1). Hence, uniqueness of (5.3) follows from uniqueness of (2.1).

A recursive argument, relying on differentiability properties of solutions of linear parabolic equations, shows that $F(t), F_{t}(t)$, and therefore $\psi(t), \psi_{t}(t)$, belong to $C^{k}([0,1])$ for any $k \geq 1$. To prove that $0<\varepsilon \psi_{x}<1$ for $(t, x) \in(0, \infty) \times[0,1]$, let $\gamma:=\psi_{x}$ and observe that $\gamma$ solves the nonlinear equation with mixed boundary conditions

$$
\left\{\begin{array}{l}
\gamma_{t}=\varepsilon \gamma_{x x}+[g \gamma(1-\varepsilon \gamma)]_{x} \\
\varepsilon \gamma_{x}(t, 0)=-g(t, 0) \gamma(t, 0)(1-\varepsilon \gamma(t, 0)), \\
\varepsilon \gamma_{x}(t, 1)=-g(t, 1) \gamma(t, 1)(1-\varepsilon \gamma(t, 1)) \\
\gamma(0, \cdot)=\varphi_{x}(\cdot)
\end{array}\right.
$$

where $g=\left[e^{\psi}-1\right] /\left[e^{\psi}+1\right]$. Since $g_{x}$ is bounded on compact subsets of $(0, \infty) \times[0,1]$, and $\varphi_{x}$ is neither identically equal to 0 nor to $\varepsilon^{-1}$, Theorem 3.7 in [33] and the remark (ii) following it, imply the result.

Finally, by [15. Thm. 4.9], as $t \rightarrow \infty$, the function $F(t)$ converges to $\bar{\rho}_{\varepsilon}$ in the $C^{1}([0,1])$ topology, uniformly over $F_{0}:[0,1] \rightarrow\left[\rho_{0}, \rho_{1}\right]$. By the methods there developed, it is straightforward to prove this statement in the $C^{3}([0,1])$ topology. Since $F(t)$ converges to $\bar{\rho}_{\varepsilon}$ in the $C^{3}([0,1])$ topology uniformly in $F_{0}, \psi(t)$ converges to $\log \left[\bar{\rho}_{\varepsilon} /\left(1+\bar{\rho}_{\varepsilon}\right)\right]=\bar{\varphi}_{\varepsilon}$ in the $C^{3}([0,1])$ topology, uniformly in $\varphi$.

Recalling (4.1), fix $\rho$ in $M^{o}$ and $\varphi$ in $\mathcal{P}(\rho)$. Let $\psi$ be the solution to (5.3) and define $v:[0, \infty) \times[0,1] \rightarrow \mathbb{R}$ by

$$
v:=\frac{1}{1+e^{\psi}}-\frac{\varepsilon \psi_{x x}}{\psi_{x}\left(1-\varepsilon \psi_{x}\right)} .
$$

Since $\varphi$ is smooth, $\psi(t)$ and $v(t)$ belong to $C^{k}([0,1])$ for all $t \geq 0, k \geq 1$.

Lemma 5.5. The function $v$ defined by (5.4) is smooth, solves

$$
\left\{\begin{array}{l}
v_{t}-f(v)_{x}=\varepsilon v_{x x}-2 \varepsilon\left[\sigma(v) \psi_{x}\right]_{x} \\
v(t, 0)=\rho_{0}, \quad v(t, 1)=\rho_{1} \\
v(0, \cdot)=\rho(\cdot)
\end{array}\right.
$$

and satisfies $0<v<1,(t, x) \in[0, \infty) \times[0,1]$. Moreover, as $t \rightarrow+\infty$ the function $v(t)$ converges to $\bar{\rho}_{\varepsilon}$ in the $C^{1}([0,1])$ topology, uniformly for $\rho$ in $M^{o}$.

Proof. By using the differential equation in (5.3) and the identity $f(u)=\sigma(u)=$ $u(1-u)$, a tedious computation shows that $v$ solves the differential equation in (5.5). The boundary conditions in (5.5) follow directly from (5.3) and the definition of $v$. The initial condition in (5.5) holds, in view of (5.4), because $\varphi$ is a fixed point of $K_{\rho}$ and therefore solves the Euler-Lagrange equation (2.11). Since the boundary conditions are bounded away from 0 and 1 , and since $\psi_{x x}$ is bounded on compact subsets of $[0, \infty) \times[0,1]$, by Theorem 3.7 in 33 and the remark (ii) following it, $0<v<1,(t, x) \in[0, \infty) \times[0,1]$.

By Lemma [5.4 $\psi(t)$ converges in the $C^{3}([0,1])$ topology to $\log \left[\bar{\rho}_{\varepsilon} /\left(1-\bar{\rho}_{\varepsilon}\right)\right]$, uniformly in $\varphi$. Therefore, by (5.4), $v(t)$ converges in the $C^{1}([0,1])$ topology to $\bar{\rho}_{\varepsilon}$ uniformly for $\rho$ in $M^{o}$. 
Lemma 5.6. Fix a time interval $\left[T_{1}, T_{2}\right]$, a smooth path $u \in C\left(\left[T_{1}, T_{2}\right] ; M^{o}\right)$, and a smooth path $\varphi \in C\left(\left[T_{1}, T_{2}\right] ; \mathcal{F}\right)$ with $0<\varepsilon \varphi_{x}<1$. Then,

$$
\begin{aligned}
\mathcal{G}_{\varepsilon}(u & \left.\left(T_{2}\right), \varphi\left(T_{2}\right)\right)-\mathcal{G}_{\varepsilon}\left(u\left(T_{1}\right), \varphi\left(T_{1}\right)\right) \\
\quad=\int_{T_{1}}^{T_{2}} & \left\{\left\langle s^{\prime}(u)-\varphi, u_{t}\right\rangle-\left\langle\frac{\varepsilon \varphi_{x x}}{\varphi_{x}\left(1-\varepsilon \varphi_{x}\right)}-\frac{1}{1+e^{\varphi}}+u, \varphi_{t}\right\rangle\right\} d t .
\end{aligned}
$$

The proof relies on a simple computation and it is omitted. We remark that if the paths $u$ and $\varphi$ are chosen so that $(\varphi(t), u(t)) \in \Sigma, t \in\left[T_{1}, T_{2}\right]$, then the second term on the right hand side of (5.6) vanishes. Therefore, the previous lemma provides an explicit expression for the integral of the symplectic one-form along any path that lies in $\Sigma$, showing in particular that the result depends only on the endpoints of the path, namely that $\Sigma$ is Lagrangian.

In view of Lemma 5.5, the time reversal of the function $v$ defined in (5.4) can be chosen as a test path for the variational problem (2.7). In the next lemma we compute the action of such a path.

Lemma 5.7. Fix a function $\rho$ in $M^{o}$ and $\varphi$ in $\mathcal{P}(\rho)$. Let $v$ be the path (5.4) and let $u \in \mathcal{U}\left(\bar{\rho}_{\varepsilon}\right)$ be defined by $u(t):=v(-t)$. Then, $I(u)=\mathcal{G}_{\varepsilon}(\rho, \varphi)-S_{\varepsilon}^{o}\left(\bar{\rho}_{\varepsilon}\right)$.

Proof. By Lemma [5.5, $u$ is a smooth path in $C\left((-\infty, 0] ; M^{\circ}\right)$, satisfies the final condition $u(0, \cdot)=\rho(\cdot)$, and solves

$$
u_{t}+f(u)_{x}=-\varepsilon u_{x x}+2 \varepsilon\left[\sigma(u) \psi_{x}^{*}\right]_{x},
$$

where $\psi^{*}(t)=\psi(-t), t \leq 0$. Equivalently, by setting $K=s^{\prime}(u)-\psi^{*}, u$ solves

$$
u_{t}+f(u)_{x}=\varepsilon u_{x x}-2 \varepsilon\left[\sigma(u) K_{x}\right]_{x} .
$$

Fix $T>0$, recall that $u$ satisfies the boundary conditions $u(t, 0)=\rho_{0}, u(t, 1)=\rho_{1}$, $t \in[-T, 0]$, and observe that $K$ is smooth and satisfies $K(t, 0)=K(t, 1)=0$, $t \in[-T, 0]$. In particular, $K \in \mathfrak{H}_{0}^{1}(\sigma(u))$ and therefore, by Theorem 2.1

$$
I_{[-T, 0]}(u)=\varepsilon\|K\|_{1, \sigma(u)}^{2}=\varepsilon\left\langle\left\langle K_{x}, \sigma(u) K_{x}\right\rangle\right\rangle
$$

Recall the definition (4.2) of the Hamiltonian $\mathbb{H}$. Multiplying both sides of (5.7) by $K$ and integrating, we get that

$$
\left\langle K, u_{t}\right\rangle-\mathbb{H}(u, K)=\varepsilon\left\langle K_{x}, \sigma(u) K_{x}\right\rangle
$$

so that

$$
I_{[-T, 0]}(u)=\int_{-T}^{0}\left\{\left\langle s^{\prime}(u)-\psi^{*}, u_{t}\right\rangle-\mathbb{H}\left(u, s^{\prime}(u)-\psi^{*}\right)\right\} d t .
$$

Since $v$ is defined by (5.4), $u$ is given by the same equation with $\psi^{*}$ replacing $\psi$. In particular, by item (iii) in Proposition $4.1 \mathbb{H}\left(u, s^{\prime}(u)-\psi^{*}\right)$ vanishes. Hence, by Lemma 5.6 .

$$
I_{[-T, 0]}(u)=\int_{-T}^{0}\left\langle s^{\prime}(u)-\psi^{*}, u_{t}\right\rangle d t=\mathcal{G}_{\varepsilon}(\rho, \varphi)-\mathcal{G}_{\varepsilon}(v(T), \psi(T)) .
$$

By Lemma 5.4, $\psi(T)$ converges to $s^{\prime}\left(\bar{\rho}_{\varepsilon}\right)$ in the $C^{3}([0,1])$ topology and by Lemma 5.5. $v(T)$ converges to $\bar{\rho}_{\varepsilon}$ in the $C^{1}([0,1])$ topology. Therefore, $\mathcal{G}_{\varepsilon}(v(T), \psi(T))$ converges to $\mathcal{G}_{\varepsilon}\left(\bar{\rho}_{\varepsilon}, s^{\prime}\left(\bar{\rho}_{\varepsilon}\right)\right)$ which is equal to $S_{\varepsilon}^{o}\left(\bar{\rho}_{\varepsilon}\right)$ by (4.5). In conclusion,

$$
I(u)=\lim _{T \rightarrow \infty} I_{[-T, 0]}(u)=\mathcal{G}_{\varepsilon}(\rho, \varphi)-S_{\varepsilon}^{o}\left(\bar{\rho}_{\varepsilon}\right),
$$

which proves the lemma. 
It follows from the previous lemma that if $\varphi$ is chosen in $\mathcal{F}(\rho)$, namely $\varphi$ is a minimizer for $\mathcal{G}_{\varepsilon}(\rho, \cdot)$, then $I(u)=S_{\varepsilon}^{o}(\rho)-S_{\varepsilon}^{o}\left(\bar{\rho}_{\varepsilon}\right)=S_{\varepsilon}(\rho)$. In particular, this proves the inequality $\widehat{V}_{\varepsilon}(\rho) \leq S_{\varepsilon}(\rho)$ for smooth functions $\rho$ in $M^{o}$, By a density argument, we next show this bound holds for any $\rho \in M$.

Proof of Theorem 2.3; the bound $\widehat{V}_{\varepsilon} \leq S_{\varepsilon}$. Fix $\rho \in M$ and denote by $\varphi \in \mathcal{F}(\rho)$, $\left\{\rho^{n}\right\} \subset M^{o}$, and $\left\{\varphi^{n}\right\} \subset \mathcal{F}$ the function and the sequences provided by Lemma 4.5. Denote by $\psi$ the classical solution to (5.3), and define $u:(-\infty, 0] \times[0,1] \rightarrow[0,1]$ by

$$
u(t)= \begin{cases}\rho & \text { if } t=0, \\ v(-t) & \text { if } t<0,\end{cases}
$$

where $v$ is the function defined in (5.4). Since $\varphi \in C^{1}([0,1]), \psi(t) \rightarrow \varphi$ in the $C^{1}$ topology as $t \downarrow 0$. Since by Lemma 4.3 we have $0<\varepsilon \varphi_{x}<1$, it is simple to check that the function $u(t)$ converges to $\rho$ in $M$ as $t \uparrow 0$. Hence, by the convergence of $\psi(t)$ as $t \rightarrow+\infty$ stated in Lemma [5.4, the path $u$ belongs to the set $\mathcal{U}\left(\bar{\rho}_{\varepsilon}\right)$ introduced in (2.5).

Let $\psi^{n}$ be the solution to (5.3) with $\varphi$ replaced by $\varphi^{n}$ and $v^{n}$ be as defined in (5.4) with $\psi$ replaced by $\psi^{n}$. Let finally $u^{n}:(-\infty, 0] \times[0,1] \rightarrow[0,1]$ be defined by $u^{n}(t)=v^{n}(-t)$. In view of the continuity with respect to the initial condition of the solution to the viscous Burgers equation (2.1) and the uniformity of the convergence as $t \rightarrow+\infty$ stated in Lemma [5.5, the sequence $\left\{u^{n}\right\}$ converges to $u$ in $\mathcal{U}\left(\bar{\rho}_{\varepsilon}\right)$. The lower semicontinuity of the functional $I: \mathcal{U}\left(\bar{\rho}_{\varepsilon}\right) \rightarrow[0,+\infty]$, together with Lemmata 5.7 and 4.5 . now imply

$$
I(u) \leq \liminf _{n} I\left(u^{n}\right)=\liminf _{n}\left[\mathcal{G}_{\varepsilon}\left(\rho^{n}, \varphi^{n}\right)-S_{\varepsilon}^{o}\left(\bar{\rho}_{\varepsilon}\right)\right]=\mathcal{G}_{\varepsilon}(\rho, \varphi)-S_{\varepsilon}^{o}\left(\bar{\rho}_{\varepsilon}\right)=S_{\varepsilon}(\rho),
$$

whence $\widehat{V}_{\varepsilon}(\rho) \leq S_{\varepsilon}(\rho)$ and the proof is concluded.

Lower bound for the quasi-potential. Before carrying out the details, we explain the main idea and the novel difficulty here encountered. Fix $\rho \in M, T>0$ and a path $u \in C([-T, 0] ; M)$ such that $u(-T)=\bar{\rho}_{\varepsilon}$ and $u(0)=\rho$. We need to show $I_{[-T, 0]}(u) \geq S_{\varepsilon}(\rho)$. Assume that the path $u$ is smooth, bounded away from zero and one, and satisfies the boundary conditions $\rho_{0}, \rho_{1}$ at the endpoints of $[0,1]$. By the variational definition (2.3) of the action functional and the definition (4.2) of the Hamiltonian $\mathbb{H}$, for each smooth function $H:[-T, 0] \times[0,1] \rightarrow \mathbb{R}$ vanishing at the boundary, we have

$$
\begin{aligned}
I_{[-T, 0]}(u) & \geq\left\langle\left\langle u_{t}+f(u)_{x}-\varepsilon u_{x x}, H\right\rangle\right\rangle-\varepsilon\left\langle\left\langle H_{x}, \sigma(u) H_{x}\right\rangle\right\rangle \\
& =\int_{-T}^{0}\left[\left\langle H, u_{t}\right\rangle-\mathbb{H}(u, H)\right] d t .
\end{aligned}
$$

Assume now that for each $t \in[-T, 0]$ there exists a unique solution $\varphi(t)$ to the Euler-Lagrange equation (2.11) with $\rho$ replaced by $u(t)$. Assume furthermore that $\varphi(t)$ is smooth; in this case we may choose above $H=s^{\prime}(u)-\varphi$. In view of item (iii) in Proposition 4.1 and Lemma 5.6 we then conclude $I_{[-T, 0]}(u) \geq S_{\varepsilon}(\rho)$. If for each $\rho \in M$ the functional $\mathcal{G}(\rho, \cdot)$ has a unique critical point, it is not difficult to turn the previous argument into a proof [6, 8]. On the other hand, in the case here discussed we have to face the lack of uniqueness of (2.11): if we choose the "wrong" $\varphi$, the bound we would get by the previous argument will not be sharp. We shall overcome this problem by discretizing the time interval $[-T, 0]$ and choosing 
a piecewise constant path $\varphi(t)$. As we show, the previous argument gives then the sharp bound provided we choose the optimal $\varphi$ at the endpoint of each time step.

We start by recalling the following density result, which is proven in Theorem 5.1 of 10 .

Lemma 5.8. Fix $T>0$ and a path $u$ in $C([-T, 0] ; M)$ such that $u(-T)=\bar{\rho}_{\varepsilon}$ and $I_{[-T, 0]}(u)<\infty$. There exists a sequence $\left\{u^{n}\right\} \subset C([-T, 0] ; M)$ of smooth functions $u^{n}:[-T, 0] \times[0,1] \rightarrow(0,1)$ converging to $u$ in $C([-T, 0] ; M)$ such that $u^{n}(-T, \cdot)=\bar{\rho}_{\varepsilon}(\cdot), u^{n}(t, 0)=\rho_{0}, u^{n}(t, 1)=\rho_{1}, t \in[-T, 0]$, and $I_{[-T, 0]}\left(u^{n}\right)$ converges to $I_{[-T, 0]}(u)$.

Proof of Theorem 2.3: the bound $S_{\varepsilon} \leq V_{\varepsilon}$. Fix a function $\rho$ in $M, T>0$ and a path $u$ in $C([-T, 0] ; M)$ such that $u(-T)=\bar{\rho}_{\varepsilon}, u(0)=\rho$. We need to show that $I_{[-T, 0]}(u) \geq S_{\varepsilon}(\rho)$.

Assume firstly that $\rho$ belongs to $M^{o}$ and that $u:[-T, 0] \times[0,1] \rightarrow[0,1]$ is a smooth path bounded away from zero and one and satisfies $u(t, 0)=\rho_{0}, u(t, 1)=\rho_{1}$, $t \in[-T, 0]$. In this case, as we have seen above, (5.9) holds for any $H$ in $\mathfrak{H}_{0}^{1}(\sigma(u))$. Consider a partition $[-T, 0)=\bigcup_{k=1}^{n}\left[-T_{k},-T_{k-1}\right)$ with $T_{0}=0$ and $T_{n}=T$. For $k=1, \cdots, n$, choose $\varphi^{k} \in \mathcal{F}\left(u\left(-T_{k}\right)\right)$ namely, $\varphi^{k}$ is a minimizer for $\mathcal{G}_{\varepsilon}\left(u\left(-T_{k}\right), \cdot\right)$. In view of Lemma 4.2 $\varphi^{k}$ belongs also to $\mathcal{P}\left(u\left(-T_{k}\right)\right)$. Define the path $\varphi$ piecewise constant in $[-T, 0]$ by $\varphi(t)=\varphi^{k}$ for $t \in\left[-T_{k},-T_{k-1}\right), k=1, \cdots, n$ and $\varphi(0)=\varphi^{1}$.

Since the path $u$ is smooth, by the definition of $\varphi, \varphi(t, 0)=\varphi_{0}, \varphi(t, 1)=\varphi_{1}$, $t \in[-T, 0]$, and $s^{\prime}(u)-\varphi$ is a smooth function in space, piecewise smooth in time which vanishes at the endpoints of $[0,1]$. In particular, $s^{\prime}(u)-\varphi$ belongs to $\mathfrak{H}_{0}^{1}(\sigma(u))$. By choosing $H=s^{\prime}(u)-\varphi$ in (5.9) we then get

$$
I_{[-T, 0]}(u) \geq \sum_{k=1}^{n} \int_{-T_{k}}^{-T_{k-1}}\left[\left\langle s^{\prime}(u)-\varphi^{k}, u_{t}\right\rangle-\mathbb{H}\left(u, s^{\prime}(u)-\varphi^{k}\right)\right] d t .
$$

By the choice of $\varphi^{k}$ and item (iii) in Proposition 4.1. $\mathbb{H}\left(u(t), s^{\prime}(u(t))-\varphi^{k}\right)$ vanishes for $t=-T_{k}$. A simple computation, based on the bounds stated in item (ii) of Proposition 4.1, shows that the map $\left[-T_{k},-T_{k-1}\right) \ni t \mapsto \mathbb{H}\left(u(t), s^{\prime}(u(t))-\varphi^{k}\right)$ is Lipschitz with a Lipschitz constant depending on $u$ but independent of $\varphi^{k}$. Hence,

$$
\lim _{n \rightarrow \infty} \sum_{k=1}^{n} \int_{-T_{k}}^{-T_{k-1}} \mathbb{H}\left(u, s^{\prime}(u)-\varphi^{k}\right) d t=0
$$

provided the mesh of the partition vanishes as $n \rightarrow \infty$.

On the other hand, since $\varphi(t)$ is constant in the interval $\left[-T_{k},-T_{k-1}\right)$, Lemma 5.6 yields

$$
\sum_{k=1}^{n} \int_{-T_{k}}^{-T_{k-1}}\left\langle s^{\prime}(u(t))-\varphi^{k}, u_{t}\right\rangle d t=\sum_{k=1}^{n}\left[\mathcal{G}_{\varepsilon}\left(u\left(-T_{k-1}\right), \varphi^{k}\right)-\mathcal{G}_{\varepsilon}\left(u\left(-T_{k}\right), \varphi^{k}\right)\right] .
$$

Since $\varphi^{k-1} \in \mathcal{F}\left(u\left(-T_{k-1}\right)\right)$, it follows $\mathcal{G}_{\varepsilon}\left(u\left(-T_{k-1}\right), \varphi^{k}\right) \geq \mathcal{G}_{\varepsilon}\left(u\left(-T_{k-1}\right), \varphi^{k-1}\right)$. The previous expression is thus bounded below by the telescopic sum

$$
\sum_{k=1}^{n}\left[\mathcal{G}_{\varepsilon}\left(u\left(-T_{k-1}\right), \varphi^{k-1}\right)-\mathcal{G}_{\varepsilon}\left(u\left(-T_{k}\right), \varphi^{k}\right)\right]=\mathcal{G}_{\varepsilon}\left(u(0), \varphi^{1}\right)-\mathcal{G}_{\varepsilon}\left(u(-T), \varphi^{n}\right) .
$$

Since $u(-T)=\bar{\rho}_{\varepsilon}$, by the choice of $\varphi^{n}$, we have $\mathcal{G}_{\varepsilon}\left(\bar{\rho}_{\varepsilon}, \varphi^{n}\right)=\inf _{\psi} \mathcal{G}_{\varepsilon}\left(\bar{\rho}_{\varepsilon}, \psi\right)=$ $S_{\varepsilon}^{o}\left(\bar{\rho}_{\varepsilon}\right)$. On the other hand, since $u(0)=\rho$, we have $\mathcal{G}_{\varepsilon}\left(u(0), \varphi^{1}\right)=\mathcal{G}_{\varepsilon}\left(\rho, \varphi^{1}\right) \geq$ 
$S_{\varepsilon}^{o}(\rho)$. By taking the limit $n \rightarrow \infty$, the previous bounds imply

$$
I_{[-T, 0]}(u) \geq S_{\varepsilon}^{o}(\rho)-S_{\varepsilon}^{o}\left(\bar{\rho}_{\varepsilon}\right)=S_{\varepsilon}(\rho) .
$$

Let now $\rho \in M$ be arbitrary and consider an arbitrary path $u \in C([-T, 0] ; M)$ such that $u(0)=\rho$ and $u(-T)=\bar{\rho}_{\varepsilon}$. Since we can assume $I_{[-T, 0]}(u)<\infty$, by Lemma 5.8. there exists a sequence of smooth paths $u^{n}$ bounded away from zero and one which converges to $u$ in $C([-T, 0] ; M)$ and such that $\lim _{n} I_{[-T, 0]}\left(u^{n}\right)=$ $I_{[-T, 0]}(u)$. The lower semicontinuity of $S_{\varepsilon}$ on $M$, see Theorem 2.2] and the result for smooth paths yield

$$
I_{[-T, 0]}(u)=\lim _{n} I_{[-T, 0]}\left(u^{n}\right) \geq \liminf _{n} S_{\varepsilon}\left(u^{n}(0)\right) \geq S_{\varepsilon}(u(0))=S_{\varepsilon}(\rho),
$$

which concludes the proof.

\section{THE INVISCID LIMIT}

In this section we discuss the inviscid limit $\varepsilon \downarrow 0$. We first discuss the variational convergence of the functional $\mathcal{G}_{\varepsilon}$ to $\mathcal{G}$. By analyzing the limiting variational problem (2.16) and using a perturbation argument we then show, provided $\varepsilon$ is small enough, that there exists $\rho \in M$ such that $\mathcal{G}_{\varepsilon}(\rho, \cdot)$ admits at least two minimizers.

Variational convergence of $\mathcal{G}_{\varepsilon}$. By standard properties of $\Gamma$-convergence, Theorems 2.6 and 2.7 are corollaries of the following result.

Theorem 6.1. Let $\mathcal{G}_{\varepsilon}: M \times \mathcal{F} \rightarrow(-\infty,+\infty]$ be the functional defined in (2.10). As $\varepsilon \downarrow 0$, the family $\left\{\mathcal{G}_{\varepsilon}\right\}_{\varepsilon>0} \Gamma$-converges to the functional $\mathcal{G}$ defined in (2.15).

Proof. We start by showing the $\Gamma$-lim inf inequality. Fix a sequence $\varepsilon_{n} \downarrow 0,(\rho, \varphi) \in$ $M \times \mathcal{F}$, and a sequence $\left\{\left(\rho^{n}, \varphi^{n}\right)\right\} \subset M \times \mathcal{F}$ converging to $(\rho, \varphi)$. We need to show $\lim \inf _{n} \mathcal{G}_{\varepsilon_{n}}\left(\rho^{n}, \varphi^{n}\right) \geq \mathcal{G}(\rho, \varphi)$. The convexity of the real function $s$ trivially implies $\liminf \operatorname{in}_{n} \int_{0}^{1} s\left(\rho^{n}\right) d x \geq \int_{0}^{1} s(\rho) d x$. Likewise, by Jensen inequality, $\int_{0}^{1} s\left(\varepsilon_{n} \varphi_{x}^{n}\right) d x \geq$ $s\left(\varepsilon_{n}\left[\varphi_{1}-\varphi_{0}\right]\right)$ which vanishes as $\varepsilon_{n} \downarrow 0$. To conclude the proof, it remains to observe that the convergence of $\rho^{n}$ to $\rho$ in $M$ and the one of $\varphi^{n}$ to $\varphi$ in $\mathcal{F}$ implies the convergence of $\int_{0}^{1}\left[\left(1-\rho^{n}\right) \varphi^{n}-\log \left(1+e^{\varphi^{n}}\right)\right] d x$.

We next show the $\Gamma$-lim sup inequality. Fix a sequence $\varepsilon_{n} \downarrow 0$ and $(\rho, \varphi) \in M \times \mathcal{F}$. We need to exhibit a sequence $\left\{\left(\rho^{n}, \varphi^{n}\right)\right\} \subset M \times \mathcal{F}$ converging to $(\rho, \varphi)$ such that $\lim \sup _{n} \mathcal{G}_{\varepsilon_{n}}\left(\rho^{n}, \varphi^{n}\right) \leq \mathcal{G}(\rho, \varphi)$. Consider first the case in which $\varphi \in \mathcal{F}$ is smooth, say $C^{1}$, and satisfies $\varphi(0)=\varphi_{0}, \varphi(1)=\varphi_{1}$. We then claim a recovering sequence is simply given by the constant sequence $\left(\rho^{n}, \varphi^{n}\right)=(\rho, \varphi)$. Indeed, by the smoothness of $\varphi$, for such a sequence we have $\int_{0}^{1} s\left(\varepsilon_{n} \varphi_{x}^{n}\right) d x \rightarrow 0$. The proof is now completed by a density argument. More precisely, it is enough to observe that, given $\varphi \in \mathcal{F}$, there exists a sequence $\left\{\varphi^{k}\right\} \subset \mathcal{F}$ converging to $\varphi$ such that $\varphi^{k} \in C^{1}([0,1]), \varphi^{k}(0)=\varphi_{0}$, $\varphi^{k}(1)=\varphi_{1}$, and $\lim _{k} \mathcal{G}\left(\rho, \varphi^{k}\right)=\mathcal{G}(\rho, \varphi)$.

Next result is a straightforward consequence of the the previous proof because the sequence used in the $\Gamma$-lim sup inequality is constant in $\rho$.

Corollary 6.2. For every $\rho$ in $M, \mathcal{G}_{\varepsilon}(\rho, \cdot) \Gamma$-converges to $\mathcal{G}(\rho, \cdot)$ as $\varepsilon \downarrow 0$.

Proof of Theorem 2.7. Since $\mathcal{F}$ is compact, Theorem 2.7 follows from Theorem 6.1 and [12, Thm. 1.21].

To deduce Theorem 2.6 we only need to "project" Theorem 6.1 to the first variable. For completeness, we detail below the proof. 
Proof of Theorem 2.6. We first show that, as $\varepsilon \downarrow 0$, the family of functionals $\left\{S_{\varepsilon}^{o}\right\}_{\varepsilon>0} \Gamma$-converges to $S^{o}$. We start by proving the $\Gamma$-liminf inequality. Fix a sequence $\varepsilon_{n} \downarrow 0, \rho \in M$ and a sequence $\left\{\rho^{n}\right\} \subset M$ converging to $\rho$. In view of the compactness of $\mathcal{F}$ and the lower semicontinuity of $\mathcal{G}_{\varepsilon_{n}}\left(\rho^{n}, \cdot\right)$, there exists a sequence $\left\{\varphi^{n}\right\} \subset \mathcal{F}$ such that $S_{\varepsilon_{n}}^{o}\left(\rho^{n}\right)=\mathcal{G}_{\varepsilon_{n}}\left(\rho^{n}, \varphi^{n}\right)$. Again by the compactness of $\mathcal{F}$, by taking if necessary a subsequence, there exists $\varphi \in \mathcal{F}$ such that $\varphi^{n} \rightarrow \varphi$. By Theorem 6.1 we then deduce

$$
\liminf _{n} S_{\varepsilon_{n}}^{o}\left(\rho^{n}\right)=\liminf _{n} \mathcal{G}_{\varepsilon_{n}}\left(\rho^{n}, \varphi^{n}\right) \geq \mathcal{G}(\rho, \varphi) \geq S^{o}(\rho) .
$$

We next prove the $\Gamma$-limsup inequality. Fix a sequence $\varepsilon_{n} \downarrow 0, \rho \in M$ and choose the constant sequence $\rho^{n}=\rho$. By Corollary 6.2, the compactness of $\mathcal{F}$, and [12, Theorem. 1.21]

$$
\lim _{n} S_{\varepsilon_{n}}^{o}(\rho)=\lim _{n} \inf _{\varphi} \mathcal{G}_{\varepsilon_{n}}(\rho, \varphi)=\inf _{\varphi} \mathcal{G}(\rho, \varphi)=S^{o}(\rho)
$$

To complete the proof we need to show that $S_{\varepsilon}^{o}\left(\bar{\rho}_{\varepsilon}\right) \rightarrow S^{o}(\bar{\rho})$ as $\varepsilon \downarrow 0$. While this statement can be proven by using (4.5) and the explicit expression of $\mathcal{G}_{\varepsilon}$ and $\mathcal{G}$, we next give an argument again based on Theorem 6.1 and the fact that $\bar{\rho}_{\varepsilon}$ converges to $\bar{\rho}$ in $L^{1}([0,1])$, pointed out just before 2.15). In the case $\rho_{0}+\rho_{1}=1$, by the latter statement we mean that $\bar{\rho}_{\varepsilon}$ converges to the stationary entropic solution with a shock placed at $x=1 / 2$. Since $\bar{\rho}_{\varepsilon} \rightarrow \bar{\rho}$ in $M$, the $\Gamma$-lim inf inequality proven above yields $\liminf { }_{\varepsilon} S_{\varepsilon}^{o}\left(\bar{\rho}_{\varepsilon}\right) \geq S^{o}(\bar{\rho})$. To prove the other inequality, fix a smooth $\varphi \in \mathcal{F}$ so that $\int_{0}^{1} s\left(\varepsilon \varphi_{x}\right) d x$ vanishes as $\varepsilon \downarrow 0$. Since $\bar{\rho}_{\varepsilon}$ converges to $\bar{\rho}$ in $L^{1}([0,1]), \int_{0}^{1} s\left(\bar{\rho}_{\varepsilon}\right) d x$ converges to $\int_{0}^{1} s(\bar{\rho}) d x$. Therefore

$$
\limsup _{\varepsilon} S_{\varepsilon}^{o}\left(\bar{\rho}_{\varepsilon}\right) \leq \limsup _{\varepsilon} \mathcal{G}_{\varepsilon}\left(\bar{\rho}_{\varepsilon}, \varphi\right)=\mathcal{G}(\bar{\rho}, \varphi) .
$$

By optimizing on $\varphi$ we then deduce $\lim _{\sup _{\varepsilon}} S_{\varepsilon}^{o}\left(\bar{\rho}_{\varepsilon}\right) \leq S^{o}(\bar{\rho})$, which concludes the proof.

The variational problem appearing in (2.16) is much simpler than the one in (2.13). In fact, the former can be reduced to a one-dimensional minimum problem. More precisely, we can restrict the infimum in (2.16) to step functions $\varphi$. Denote by $\widetilde{\mathcal{G}}(\rho, y)$ the functional $\mathcal{G}(\rho, \varphi)$ defined in 2.15 evaluated at $\varphi=\varphi^{(y)}, y \in[0,1]$, where $\varphi^{(y)}(x):=\varphi_{0} \mathbf{1}_{[0, y)}(x)+\varphi_{1} \mathbf{1}_{[y, 1]}(x)$. In other words, let $\widetilde{\mathcal{G}}: M \times[0,1] \rightarrow \mathbb{R}$ be the functional defined by

$$
\begin{aligned}
\widetilde{\mathcal{G}}(\rho, y):= & \int_{0}^{1} s(\rho) d x+\varphi_{0} \int_{0}^{y}(1-\rho) d x+\varphi_{1} \int_{y}^{1}(1-\rho) d x \\
& -y \log \left(1+e^{\varphi_{0}}\right)-(1-y) \log \left(1+e^{\varphi_{1}}\right) .
\end{aligned}
$$

Proposition 6.3. Fix $\rho \in M$. Then,

$$
\inf \{\mathcal{G}(\rho, \varphi), \varphi \in \mathcal{F}\}=\inf \{\widetilde{\mathcal{G}}(\rho, y), y \in[0,1]\} .
$$

Proof. Since the functional $\mathcal{G}(\rho, \cdot)$ is concave and $\mathcal{F}$ is a compact convex set, the infimum on the left hand side of (6.1) is achieved when $\varphi$ belongs to the extremal elements of $\mathcal{F}$. We thus have to show that the extremal elements of $\mathcal{F}$ are $\left\{\varphi^{(y)}, y \in\right.$ $[0,1]\}$. This is easily proven recalling (2.8) and noticing that, given $m>0$, the extremal elements of $\mathcal{P}_{m}([0,1])$ are $\left\{m \delta_{y}, y \in[0,1]\right\}$, where $\delta_{y}$ is the Dirac measure at $y$. 
Non uniqueness of the minimizers. The statements of Theorems 2.2 and 2.5 imply that for $\varepsilon$ small enough there exists $\rho \in M$ such that $\mathcal{P}_{\varepsilon}(\rho)$ is a not a singleton. This result can however be directly proven by a simpler argument, presented below, which also shows that there exists points in $\mathcal{P}_{\varepsilon}(\rho)$ which are not in $\mathcal{F}_{\varepsilon}(\rho)$.

Lemma 6.4. Fix $\varphi_{0}<\varphi_{1}$. There exists $\varepsilon^{*} \in\left(0, \varepsilon_{0}\right)$ and a smooth function $\rho$ in $M$ such that $K_{\rho, \varepsilon}$ has at least two fixed points for $\varepsilon<\varepsilon^{*}$.

Proof. Let $\varphi \in \mathcal{F}$ be the affine function $\varphi(x)=\varphi_{0}(1-x)+\varphi_{1} x$, and let $\rho \in M$ be given by $\rho=1 /\left(1+e^{\varphi}\right)$. Clearly, $\varphi$ is a fixed point of $K_{\rho, \varepsilon}$. To conclude the proof, it is enough to show that, provided $\varepsilon$ is small enough, $\varphi$ is not a minimizer of $\mathcal{G}_{\varepsilon}(\rho, \cdot)$. Recall the second variation of $\mathcal{G}_{\varepsilon}(\rho, \cdot)$ evaluated at $\varphi$ is the quadratic form (4.3). For the above choices of $\rho$ and $\varphi$, it is easy to check that this quadratic form is not positive semi-definite for $\varepsilon$ small enough, which proves the lemma.

In order to prove Theorem 2.5 we first analyze the limiting variational problem (2.16) and characterize, for suitable functions $\rho$, its minimizers. Fix $\varphi_{0}<\varphi_{1}$, set $\bar{\varphi}:=\left(\varphi_{0}+\varphi_{1}\right) / 2$, and let

$$
\begin{gathered}
A:=1-\frac{\log \left(1+e^{\varphi_{1}}\right)-\log \left(1+e^{\varphi_{0}}\right)}{\varphi_{1}-\varphi_{0}}, \quad A_{+}:=1-\frac{\log \left(1+e^{\bar{\varphi}}\right)-\log \left(1+e^{\varphi_{0}}\right)}{\bar{\varphi}-\varphi_{0}}, \\
A_{-}:=1-\frac{\log \left(1+e^{\varphi_{1}}\right)-\log \left(1+e^{\bar{\varphi}}\right)}{\varphi_{1}-\bar{\varphi}} .
\end{gathered}
$$

Since the real function $x \mapsto \log \left(1+e^{x}\right)$ is strictly increasing, strictly convex, and Lipschitz with Lipschitz constant one, we have $0<A_{-}<A<A_{+}<1$. Fix a continuous function $\rho:[0,1] \rightarrow[0,1]$ satisfying the following condition. There exist three reals $0 \leq y_{-}<y_{0}<y_{+} \leq 1$ such that: $\rho\left(y_{0}\right)=\rho\left(y_{ \pm}\right)=A, \rho(x)<A$ for $x \in\left[0, y_{-}\right) \cup\left(y_{0}, y_{+}\right), \rho(x)>A$ for $x \in\left(y_{-}, y_{0}\right) \cup\left(y_{+}, 1\right)$, and $A_{-}<\rho(x)<A_{+}$for $x \in\left[y_{-}, y_{+}\right]$. Recalling $\mathcal{P}_{m}([0,1]), m>0$, denotes the set of positive Borel measure on $[0,1]$ with mass $m$ endowed with the topology of weak convergence, let

$$
\begin{gathered}
\mathcal{P}_{m}^{-}([0,1]):=\left\{\mu \in \mathcal{P}_{m}([0,1]): \mu\left(\left[0, y_{0}\right]\right) \geq m / 2\right\}, \\
\mathcal{P}_{m}^{+}([0,1]):=\left\{\mu \in \mathcal{P}_{m}([0,1]): \mu\left(\left[y_{0}, 1\right]\right) \geq m / 2\right\} .
\end{gathered}
$$

Note that $\mathcal{P}_{m}^{ \pm}([0,1])$ is a closed convex subset of $\mathcal{P}_{m}([0,1])$. Recalling (2.8), let accordingly

$$
\mathcal{F}^{ \pm}:=\left\{\varphi \in \mathcal{F}: \varphi(x)=\varphi_{0}+\mu([0, x]) \text { for some } \mu \in \mathcal{P}_{\varphi_{1}-\varphi_{0}}^{ \pm}([0,1])\right\}
$$

and observe that $\mathcal{F}=\mathcal{F}^{-} \cup \mathcal{F}^{+}$.

Lemma 6.5. Fix $\rho$ as above and set $\varphi_{ \pm}^{*}:=\varphi_{0} \mathbf{1}_{\left[0, y_{ \pm}\right)}+\varphi_{1} \mathbf{1}_{\left[y_{ \pm}, 1\right]}$. Then

$$
\arg \inf \left\{\mathcal{G}(\rho, \varphi), \varphi \in \mathcal{F}^{ \pm}\right\}=\left\{\varphi_{ \pm}^{*}\right\}
$$

namely, the infimum of $\mathcal{G}(\rho, \cdot)$ over $\mathcal{F}^{ \pm}$is uniquely achieved at $\varphi_{ \pm}^{*}$. If furthermore $\rho$ satisfies $\int_{y_{-}}^{y_{+}} \rho d x=A\left(y_{+}-y_{-}\right)$then $\inf _{\mathcal{F}^{-}} \mathcal{G}(\rho, \cdot)=\inf _{\mathcal{F}^{+}} \mathcal{G}(\rho, \cdot)$.

This result implies that if the function $\rho$ satisfies all conditions of the previous lemma then

$$
\arg \inf \{\mathcal{G}(\rho, \varphi), \varphi \in \mathcal{F}\}=\left\{\varphi_{-}^{*}, \varphi_{+}^{*}\right\}
$$

namely, the functional $\mathcal{G}(\rho, \cdot)$ on $\mathcal{F}$ has exactly two minimizers. In fact, by using Proposition 6.3. this can be easily proven even if the condition $A_{-}<\rho(x)<A_{+}$, $x \in\left[y_{-}, y_{+}\right]$, is dropped. We also mention that, as observed in [17, if $\rho$ is constant 
and equal to $A$, then there exists a one parameter family of minimizers for $\mathcal{G}(\rho, \cdot)$ which is exactly the collection of the extremal elements $\left\{\varphi^{(y)}, y \in[0,1]\right\}$ of $\mathcal{F}$.

Proof of Lemma 6.5. Since $\mathcal{G}(\rho, \cdot)$ is a strictly concave functional and $\mathcal{F}^{ \pm}$is a compact convex set, the infimum can only be achieved at the extremal elements of $\mathcal{F}^{ \pm}$. These elements can be easily characterized. Indeed, it is simple to check that the extremal points of $\mathcal{P}_{m}^{-}([0,1])$ are given by $E_{1}^{-} \cup E_{2}^{-}$where

$$
E_{1}^{-}=\left\{m \delta_{x}, x \in\left[0, y_{0}\right]\right\}, \quad E_{2}^{-}=\left\{\frac{m}{2}\left(\delta_{x}+\delta_{x^{\prime}}\right), x \in\left[0, y_{0}\right], x^{\prime} \in\left[y_{0}, 1\right]\right\}
$$

We denote by $\mathcal{F}_{\mathrm{e}, 1}^{-}$and $\mathcal{F}_{\mathrm{e}, 2}^{-}$the corresponding subsets of $\mathcal{F}^{-}$with $m=\varphi_{1}-\varphi_{0}$. Note that $\varphi \in \mathcal{F}_{\mathrm{e}, 1}^{-}$iff $\varphi$ jumps from $\varphi_{0}$ to $\varphi_{1}$ at some point $x \in\left[0, y_{0}\right]$ while $\varphi \in \mathcal{F}_{\mathrm{e}, 2}^{-}$iff $\varphi$ jumps from $\varphi_{0}$ to $\bar{\varphi}$ at some point $x \in\left[0, y_{0}\right]$ and from $\bar{\varphi}$ to $\varphi_{1}$ at some point $x^{\prime} \in\left[y_{0}, 1\right]$.

We have thus reduced the original problem of the minimum over $\mathcal{F}^{-}$to a minimum problem in one and two real variables. By using that $\rho(x)<A_{+}$for $x \in\left[0, y_{0}\right]$ and $\rho(x)>A_{-}$for $x \in\left[y_{0}, 1\right]$, elementary computations show that the infimum of $\mathcal{G}(\rho, \cdot)$ over $\mathcal{F}_{\mathrm{e}, 2}^{-}$is achieved when $\varphi$ has a single jump at $y_{0}$. Note that such a $\varphi$ belongs both to $\mathcal{F}_{\mathrm{e}, 2}^{-}$and $\mathcal{F}_{\mathrm{e}, 1}^{-}$. Likewise, by using that $\rho(x)<A$ for $x \in\left[0, y_{-}\right)$and $\rho(x)>A$ for $x \in\left(y_{-}, y_{0}\right)$, it is readily seen that the infimum over $\mathcal{F}_{\mathrm{e}, 1}^{-}$is uniquely achieved at $\varphi_{-}^{*}$.

The argument for $\mathcal{F}^{+}$is the same and the last statement follows from a direct computation.

By using the variational convergence of $\mathcal{G}_{\varepsilon}$ to $\mathcal{G}$ and the above lemma, we finally show, provided $\varepsilon$ is small enough and $\rho$ is suitably chosen, the functional $\mathcal{G}_{\varepsilon}(\rho, \cdot)$ admits more than a single minimizer.

Proof of Theorem 2.5. Fix a continuous function $\rho \in M$ bounded away from zero and one satisfying the condition stated above Lemma 6.5 as well as the condition in the last statement of that lemma. Pick now a continuous positive function $\lambda:[0,1] \rightarrow \mathbb{R}_{+}$such that $\operatorname{supp} \lambda \subset\left(y_{-}, y_{0}\right)$ and $\int_{0}^{1} d x \lambda=1$. Choose finally $\delta>0$ so small that for each $\alpha \in[-\delta, \delta]$ the function $\rho^{(\alpha)}:=\rho+\alpha \lambda$ still satisfies the condition stated above Lemma 6.5 with $y_{0}$ and $y_{ \pm}$independent on $\alpha$. Note however that for $\alpha \neq 0$ the functional $\mathcal{G}\left(\rho^{(\alpha)}, \cdot\right)$ has a unique minimizer. The proof of the theorem will be accomplished by considering the one parameter family of functions $\left\{\rho^{(\alpha)},|\alpha| \leq \delta\right\}$ and showing that, for $\varepsilon$ small enough, there exists $\alpha_{0}$ for which $\mathcal{G}_{\varepsilon}\left(\rho^{\left(\alpha_{0}\right)}, \cdot\right)$ has at least two distinct minimizers.

Given $\varepsilon>0$, let $g_{\varepsilon}:[-\delta, \delta] \rightarrow \mathbb{R}$ be defined by

$$
g_{\varepsilon}(\alpha):=\inf \left\{\mathcal{G}_{\varepsilon}\left(\rho^{(\alpha)}, \varphi\right), \varphi \in \mathcal{F}^{+}\right\}-\inf \left\{\mathcal{G}_{\varepsilon}\left(\rho^{(\alpha)}, \varphi\right), \varphi \in \mathcal{F}^{-}\right\}
$$

and observe that, in view of Lemma 4.4, the function $g_{\varepsilon}$ is continuous. Let int $\mathcal{F}^{ \pm}$ be the interior of $\mathcal{F}^{ \pm}$. By Theorem 6.1 and standard properties of $\Gamma$-convergence, see e.g. [12, Prop. 1.18], for each $\alpha \in[-\delta, \delta]$

$$
\inf _{\mathcal{F}^{ \pm}} \mathcal{G}\left(\rho^{(\alpha)}, \cdot\right) \leq \liminf _{\varepsilon \downarrow 0} \inf _{\mathcal{F}^{ \pm}} \mathcal{G}_{\varepsilon}\left(\rho^{(\alpha)}, \cdot\right) \leq \limsup _{\varepsilon \downarrow 0} \inf _{\operatorname{int} \mathcal{F}^{ \pm}} \mathcal{G}_{\varepsilon}\left(\rho^{(\alpha)}, \cdot\right) \leq \inf _{\operatorname{int} \mathcal{F}^{ \pm}} \mathcal{G}\left(\rho^{(\alpha)}, \cdot\right)
$$

Whence, using $\varphi_{ \pm}^{*} \in \operatorname{int} \mathcal{F}^{ \pm}$and Lemma 6.5.

$$
\lim _{\varepsilon \downarrow 0} \inf _{\varphi \in \mathcal{F}_{ \pm}} \mathcal{G}_{\varepsilon}\left(\rho^{(\alpha)}, \varphi\right)=\mathcal{G}\left(\rho^{(\alpha)}, \varphi_{ \pm}^{*}\right)
$$


so that

$$
\begin{aligned}
\lim _{\varepsilon \downarrow 0} g_{\varepsilon}(\delta) & =\mathcal{G}\left(\rho^{(\delta)}, \varphi_{+}^{*}\right)-\mathcal{G}\left(\rho^{(\delta)}, \varphi_{-}^{*}\right) \\
& =\mathcal{G}\left(\rho, \varphi_{+}^{*}\right)-\mathcal{G}\left(\rho, \varphi_{-}^{*}\right)-\delta \int_{0}^{1} \lambda\left(\varphi_{+}^{*}-\varphi_{-}^{*}\right) d x=\delta\left(\varphi_{1}-\varphi_{0}\right)
\end{aligned}
$$

In particular, there exists $\varepsilon_{1} \in\left(0, \varepsilon_{0}\right)$ such that for any $\varepsilon \in\left(0, \varepsilon_{1}\right)$ we have $g_{\varepsilon}(\delta)>0$ and, by the same argument, $g_{\varepsilon}(-\delta)<0$.

Applying the theorem on the existence of zeros for a continuous function of a real variable, we deduce that for each $\varepsilon \in\left(0, \varepsilon_{1}\right)$ there exists $\alpha_{0} \in(-\delta, \delta)$ such that $g_{\varepsilon}\left(\alpha_{0}\right)=0$. This implies the existence of at least two distinct minimizers for $\mathcal{G}_{\varepsilon}\left(\rho^{\left(\alpha_{0}\right)}, \cdot\right)$. Note indeed that although the sets $\mathcal{F}^{+}$and $\mathcal{F}^{-}$are not disjoint, the minimizer of $\mathcal{G}_{\varepsilon}\left(\rho^{\left(\alpha_{0}\right)}, \cdot\right)$ over $\mathcal{F}^{-}$cannot coincide with the one over $\mathcal{F}^{+}$since they respectively converge to $\varphi_{+}^{*}$ and $\varphi_{-}^{*}$ as $\varepsilon \downarrow 0$. The last statement follows again from Theorem 6.1 and standard properties of $\Gamma$-convergence, see e.g. [12, Thm. 1.21].

\section{HAMILTON-JACOBI EQUATION}

In the context of diffusion processes in $\mathbb{R}^{n}$, the quasi-potential is connected to a Hamilton-Jacobi equation. More precisely, let $I$ be the action functional corresponding to the Lagrangian $\mathbb{L}(x, \dot{x})$ and denote by $\mathbb{H}(x, p)$ the associated Hamiltonian. Under suitable conditions, the quasi-potential, as defined in (1.2), is then a viscosity solution to the Hamilton-Jacobi equation $\mathbb{H}(x, D v)=0$ [13, 32. In an infinite dimensional setting, the theory of Hamilton-Jacobi equation, in particular of stationary Hamilton-Jacobi equation, is much less developed. As Theorem 2.3 gives a somewhat explicit expression for the quasi-potential, the problem here discussed may reveal itself to be a good example for the development of the theory of infinite dimensional Hamilton-Jacobi equations. In this section, we present some possible formulations of the Hamilton-Jacobi equation which seem apt for the variational problems (2.4) or 2.7).

It is not clear how to introduce a differentiable structure on the set $M$ in such a way that the Hamiltonian $\mathbb{H}$ in (4.2) becomes a well defined function on the cotangent bundle of $M$. The formalization of the Hamilton-Jacobi equation is thus a non trivial issue. In the following we consider two possibilities to circumvent this problem. In the first we simply consider the subset of $M$ given by the smooth functions $\rho:[0,1] \rightarrow[0,1]$ which are bounded away from zero and one and satisfy the boundary conditions. In the second one we exploit the symplectic transformation (3.5) and deduce the functional $\Lambda^{*}$, recall (4.7) and (4.9), solves the Hamilton-Jacobi equation with the Hamiltonian $\widetilde{\mathbb{H}}$ introduced in $(3.6)$.

Recall the definitions (2.13), (4.1), and (4.2). We would like to claim that the functional $S_{\varepsilon}^{o}$ solves the Hamilton-Jacobi equation $\mathbb{H}(\rho, D U)=0$ in $M^{o}$. In this setting, we formulate the notion of viscosity solutions to such Hamilton-Jacobi equation in terms of Gâteaux sub-differentials, recall (4.10).

Theorem 7.1. For each $\rho \in M^{o}$ we have $D_{\mathrm{G}}^{ \pm} S_{\varepsilon}^{o}(\rho) \subset \mathcal{H}_{0}^{1}$. Moreover, $S_{\varepsilon}^{o}$ is a Gâteaux viscosity solution to $\mathbb{H}(\rho, D U)=0$ in $M^{o}$ namely, the two following inequalities hold for any $\rho \in M^{\circ}$

$$
\begin{array}{ll}
\mathbb{H}(\rho, h) \leq 0, & \forall h \in D_{\mathrm{G}}^{+} S_{\varepsilon}^{o}(\rho), \\
\mathbb{H}(\rho, h) \geq 0, & \forall h \in D_{\mathrm{G}}^{-} S_{\varepsilon}^{o}(\rho) .
\end{array}
$$


Proof. Recall the decomposition (4.9) and fix $\rho \in M^{o}$. It is straightforward to check that $\mathcal{S}$ is Gauteaux differentiable at $\rho$ and $D_{\mathrm{G}} \mathcal{S}(\rho)=s^{\prime}(\rho)$. In view of Proposition 4.6 we deduce that $D_{\mathrm{G}}^{+} S_{\varepsilon}^{o}(\rho)=s^{\prime}(\rho)-\operatorname{co} \mathcal{F}(\rho)$ and

$$
D_{\mathrm{G}}^{-} S_{\varepsilon}^{o}(\rho)= \begin{cases}s^{\prime}(\rho)-\varphi & \text { if } \mathcal{F}(\rho)=\{\varphi\} \text { for some } \varphi \in \mathcal{F} \\ \varnothing & \text { otherwise }\end{cases}
$$

By Lemma 4.2 and item (ii) in Proposition 4.1, we then deduce $D_{\mathrm{G}}^{ \pm} S_{\varepsilon}^{o}(\rho) \subset \mathcal{H}_{0}^{1}$.

By item (iii) in Proposition 4.1 $\mathbb{H}\left(\rho, s^{\prime}(\rho)-\varphi\right)=0$ for any $\rho \in M^{o}$ and any $\varphi \in \mathcal{P}(\rho)$. In particular, again by Lemma 4.2 this equation holds for any $\rho \in M^{o}$ and $\varphi \in \mathcal{F}(\rho)$. The explicit expression of the Gâteaux sub-differentials above and the convexity of $\mathbb{H}(\rho, \cdot)$ on $\mathcal{H}_{0}^{1}$ now easily yield the statements.

Since in our case $\bar{\rho}_{\varepsilon}$ is the unique, globally attractive, fixed point of the flow defined by (2.1), we can try to characterize the quasi-potential $V_{\varepsilon}$, as defined in (2.4), as the maximal viscosity sub-solution of the Hamilton-Jacobi equation $\mathbb{H}(\rho, D U)=0$ satisfying $U\left(\bar{\rho}_{\varepsilon}\right)=0$. Our next result, which does not depend on the special form of the flux $f$ and mobility $\sigma$, goes in this direction.

Theorem 7.2. Let $U_{\varepsilon}: M \rightarrow \mathbb{R}$ be a lower semicontinuous functional such that $U_{\varepsilon}\left(\bar{\rho}_{\varepsilon}\right)=0$ and satisfying the following condition. For each $T>0$ and each smooth path $u:[-T, 0] \times[0,1] \rightarrow(0,1)$ such that $u(t, 0)=\rho_{0}, u(t, 1)=\rho_{1}, t \in[-T, 0]$, consider a partition $[-T, 0)=\bigcup_{k=1}^{n}\left[-T_{k},-T_{k-1}\right)$ with $T_{0}=0$ and $T_{n}=T$. Then there are elements $h^{k} \in \mathcal{H}_{0}^{1}, k=1, \ldots, n$ which are uniformly bounded in $\mathcal{H}_{0}^{1}$ and such that

$$
\mathbb{H}\left(u\left(-T_{k}\right), h^{k}\right) \leq 0, \quad k=1, \ldots, n
$$

and

$$
\limsup _{\max \left|T_{k-1}-T_{k}\right| \rightarrow 0} \sum_{k=1}^{n}\left[U_{\varepsilon}\left(-T_{k-1}\right)-U_{\varepsilon}\left(-T_{k}\right)-\int_{-T_{k}}^{-T_{k-1}}\left\langle h^{k}, u_{t}\right\rangle d t\right] \leq 0 .
$$

Then $V_{\varepsilon} \geq U_{\varepsilon}$.

Note that condition (7.2) basically requires that $h^{k}$ belongs to the super-differential of $U_{\varepsilon}$ at the point $u\left(-T_{k}\right)$ with a uniform control over the smooth path $u(t)$, $t \in[-T, 0]$. Equation (7.1) is thus a viscosity formulation of $\mathbb{H}\left(\rho, D U_{\varepsilon}\right) \leq 0$. As a matter of fact, the proof of the above theorem is quite similar to the one of the lower bound in Theorem 2.3 proven in Section 5 and we only sketch the argument.

Proof. Let $U_{\varepsilon}: M \rightarrow \mathbb{R}$ be as in the statement of the theorem. In view of Lemma 5.8 and the lower semicontinuity of $U_{\varepsilon}$, it is enough to prove the following statement. Fix $\rho \in M^{o}, T>0$, and a smooth path $u \in C\left([-T, 0] ; M^{o}\right)$ such that $u(-T)=\bar{\rho}_{\varepsilon}$, $u(0)=\rho$; then $I_{[-T, 0]}(u) \geq U_{\varepsilon}(\rho)$.

Consider a partition $[-T, 0)=\bigcup_{k=1}^{n}\left[-T_{k},-T_{k-1}\right)$ with $T_{0}=0$ and $T_{n}=T$. For $k=1, \cdots, n$, let $h^{k} \in \mathcal{H}_{0}^{1}$ as in the statement of the theorem and choose in (5.9) the piecewise constant path $H(t)=h^{k}$ for $t \in\left[-T_{k},-T_{k-1}\right), k=1, \cdots, n$ and $H(0)=h^{1}$. By assumption, $\mathbb{H}\left(u\left(-T_{k}\right), h^{k}\right) \leq 0$. Whence, by the smoothness of the path $u$ and the assumption that $\left|h^{k}\right|_{\mathcal{H}_{0}^{1}}$ is uniformly bounded,

$$
\limsup _{n \rightarrow \infty} \sum_{k=1}^{n} \int_{-T_{k}}^{-T_{k-1}} \mathbb{H}\left(u(t), h^{k}\right) d t \leq 0
$$

provided the mesh of the partition vanishes as $n \rightarrow \infty$. 
Again by assumption,

$$
\liminf _{n \rightarrow \infty} \sum_{k=1}^{n}\left\{\int_{-T_{k}}^{-T_{k-1}}\left\langle h^{k}, u_{t}\right\rangle d t-\left[U_{\varepsilon}\left(u\left(-T_{k-1}\right)\right)-U_{\varepsilon}\left(u\left(-T_{k}\right)\right)\right]\right\} \geq 0
$$

provided the mesh of the partition vanishes as $n \rightarrow \infty$. We then deduce that

$$
I_{[-T, 0]}(u) \geq U(u(0))-U(u(-T))=U(\rho),
$$

which concludes the proof.

We next discuss a formulation of the Hamilton-Jacobi equation in a strong topology and in the whole set $M$. Recalling (4.9), the basic idea is to look for an equation for $\Lambda^{*}$, equivalently to exploit the symplectic transformation (3.5).

Consider the space $L^{1}([0,1])$ equipped with the strong topology and denote by $\widehat{M}$ its (closed) subset given by $\widehat{M}:=\left\{\rho \in L^{1}([0,1]): 0 \leq \rho \leq 1\right\}$. There is nothing really peculiar with the choice of $L^{1}([0,1])$, any $L^{p}([0,1])$ with $p \in[1, \infty)$ would lead to the same results; $L^{\infty}([0,1])$ might look more natural, but its lack of separability (with respect to the strong topology) prevents its use. Let

$$
\mathcal{W}:=\left\{w \in W^{2,1}([0,1]): w(0)=\varphi_{0}, w(1)=\varphi_{0}\right\},
$$

where $W^{2,1}([0,1])$ is the Sobolev space of the functions whose second (weak) derivative belongs to $L^{1}([0,1])$. Let $\widehat{\mathbb{H}}: \widehat{M} \times \mathcal{W} \rightarrow \mathbb{R}$ be the Hamiltonian defined by

$$
\begin{aligned}
\widehat{\mathbb{H}}(\rho, w):= & -\varepsilon\left\langle w_{x}, \sigma(\rho) w_{x}\right\rangle-\varepsilon\left\langle\rho, w_{x x}\right\rangle \\
& +\left\langle\sigma(\rho), w_{x}\right\rangle-\rho_{1}\left[1-\varepsilon w_{x}(1)\right]+\rho_{0}\left[1-\varepsilon w_{x}(0)\right] .
\end{aligned}
$$

The relationship of $\widehat{\mathbb{H}}$ to the original Hamiltonian $\mathbb{H}$ is the following. Consider the anti-symplectic transformation $(\rho, h) \mapsto(\rho, w)$ where $w=s^{\prime}(\rho)-h$. The associated Hamiltonian $\widehat{\mathbb{H}}(\rho, w)=-\mathbb{H}\left(\rho, s^{\prime}(\rho)-w\right)$ is then the one defined above. We remark that while the identity $\widehat{\mathbb{H}}(\rho, w)=-\mathbb{H}\left(\rho, s^{\prime}(\rho)-w\right)$ holds only for $\rho \in M^{o}$, recall (4.1), the Hamiltonian $\widehat{\mathbb{H}}$ is defined for all $\rho$ in $\widehat{M}$.

We claim that the functional $\widehat{\Lambda}$ in (4.11) is a viscosity solution of the HamiltonJacobi equation $\widehat{\mathbb{H}}(\rho, D U)=0, \rho \in \widehat{M}$. In order to state precisely this result, we recall the notion of Fréchet sub-differentials. Let $B$ be a Banach space and denote by $B^{*}$ its dual. The norms in $B$ and $B^{*}$ are respectively denoted by $|\cdot|_{B}$ and $|\cdot|_{B^{*}}$. A function $f: B \rightarrow \mathbb{R}$ is Fréchet differentiable at $x \in B$ iff there exists $\ell \in B^{*}$ such that

$$
\lim _{y \rightarrow x} \frac{f(y)-f(x)-\langle\ell, y-x\rangle}{|y-x|_{B}}=0
$$

in this case we denote $\ell$ by $D_{\mathrm{F}} f(x)$. In general, the Fréchet sub-differential $D_{\mathrm{F}}^{-} f(x)$ and Fréchet super-differential $D_{\mathrm{F}}^{+} f(x)$ of $f$ at the point $x$ are defined as the, possibly empty, convex subsets of $B^{*}$

$$
\begin{aligned}
& D_{\mathrm{F}}^{+} f(x):=\left\{\ell \in B^{*}: \limsup _{y \rightarrow x} \frac{f(y)-f(x)-\langle\ell, y-x\rangle}{|y-x|_{B}} \leq 0\right\}, \\
& D_{\mathrm{F}}^{-} f(x):=\left\{\ell \in B^{*}: \liminf _{y \rightarrow x} \frac{f(y)-f(x)-\langle\ell, y-x\rangle}{|y-x|_{B}} \geq 0\right\} .
\end{aligned}
$$


Theorem 7.3. The functional $\widehat{\Lambda}:\left(L^{1}([0,1])\right.$, strong $) \rightarrow \mathbb{R}$ is convex and continuous, in particular locally Lipschitz. Moreover, for each $\rho \in L^{1}([0,1])$ we have $D_{\mathrm{F}}^{ \pm} \widehat{\Lambda}(\rho) \subset \mathcal{W}$. Finally, $\widehat{\Lambda}$ is a Fréchet viscosity solution of $\widehat{\mathbb{H}}(\rho, D U)=0$ in $\widehat{M}$ namely, the two following inequalities hold for any $\rho \in \widehat{M}$

$$
\begin{array}{ll}
\widehat{\mathbb{H}}(\rho, w) \leq 0, & \forall w \in D_{\mathrm{F}}^{+} \widehat{\Lambda}(\rho), \\
\widehat{\mathbb{H}}(\rho, w) \geq 0, & \forall w \in D_{\mathrm{F}}^{-} \widehat{\Lambda}(\rho) .
\end{array}
$$

Proof. Clearly $\widehat{\Lambda}$ is convex and, by the argument used in Lemma 4.4. continuous. Fix $\rho \in \widehat{M}$. Proposition 4.6 and the argument presented below Theorem 4.8 show that $D_{\mathrm{F}}^{-} \widehat{\Lambda}(\rho)=\mathrm{co} \mathcal{F}(\rho)$ and $D_{\mathrm{F}}^{+} \widehat{\Lambda}(\rho)=\{\varphi\}$ when $\mathcal{F}(\rho)=\{\varphi\}, D_{\mathrm{F}}^{+} \widehat{\Lambda}(\rho)=\varnothing$ otherwise. In particular, by the inclusion $\mathcal{F}(\rho) \subset \mathcal{P}(\rho)$ and item (ii) in Proposition 4.1. $D_{\mathrm{F}}^{ \pm} \widehat{\Lambda}(\rho) \subset \mathcal{W}$.

As in the proof of Lemma 4.3 we consider the integral operator $K_{\rho}$ defined in (2.12) also for $\rho \in L^{1}([0,1])$ and denote by $\widehat{\mathcal{P}}(\rho) \subset \mathcal{F}$ the set of fixed points of $K_{\rho}$. As in Proposition 4.1, if $\varphi \in \widehat{\mathcal{P}}(\rho)$ then $\varphi \in C^{1}([0,1])$ and there exist $\delta \in(0,1)$ and $C<\infty$, depending only on $|\rho|_{L^{1}}$, such that $\delta \leq \varepsilon \varphi_{x} \leq 1-\delta$ and $\left|\varphi_{x x}\right|_{L^{1}} \leq C$. In particular the Euler-Lagrange equation (2.11) holds a.e.

We now prove the sub-solution statement, that is $\widehat{\mathbb{H}}(\rho, w) \leq 0$ for any $\rho \in \widehat{M}$ and $w \in D_{\mathrm{F}}^{+} \widehat{\Lambda}(\rho)$. Since $D_{\mathrm{F}}^{+} \widehat{\Lambda}(\rho)=\varnothing$ if $\mathcal{F}(\rho)$ is not a singleton, we need only to consider the case $\mathcal{F}(\rho)=\{\varphi\}$ for some $\varphi \in \mathcal{F}$. Hence, as shown in the proof of Lemma 4.3. $\varphi \in \widehat{\mathcal{P}}(\rho)$. It is therefore enough to show that for any $\rho \in \widehat{M}$ and $\varphi \in \widehat{\mathcal{P}}(\rho)$ we have $\widehat{\mathbb{H}}(\rho, \varphi)=0$. This is essentially the same computation as the one used in the proof of item (iii) in Proposition 4.1. Recall $\varphi_{i}=\log \left[\rho_{i} /\left(1-\rho_{i}\right)\right]$, $i=0,1$ and observe that, in view of the bounds stated above, if $\varphi \in \widehat{\mathcal{P}}(\rho)$ then

$$
\begin{gathered}
\rho_{1}\left[1-\varepsilon \varphi_{x}(1)\right]-\rho_{0}\left[1-\varepsilon \varphi_{x}(0)\right]=\left\langle\left(\frac{e^{\varphi}}{1+e^{\varphi}}\right)_{x}, 1-\varepsilon \varphi_{x}\right\rangle-\left\langle\frac{e^{\varphi}}{1+e^{\varphi}}, \varepsilon \varphi_{x x}\right\rangle \\
=\left\langle\frac{e^{\varphi}}{\left(1+e^{\varphi}\right)^{2}}, \varphi_{x}\left(1-\varepsilon \varphi_{x}\right)\right\rangle-\left\langle\frac{e^{\varphi}}{1+e^{\varphi}}, \varepsilon \varphi_{x x}\right\rangle
\end{gathered}
$$

so that, recalling (7.3),

$$
\widehat{\mathbb{H}}(\rho, \varphi)=\left\langle\sigma(\rho)-\frac{e^{\varphi}}{\left(1+e^{\varphi}\right)^{2}}, \varphi_{x}\left(1-\varepsilon \varphi_{x}\right)\right\rangle+\left\langle\frac{e^{\varphi}}{1+e^{\varphi}}-\rho, \varepsilon \varphi_{x x}\right\rangle
$$

At this point we use the special form of $\sigma$, i.e. $\sigma(\rho)=\rho(1-\rho)$, which implies

$$
\sigma(\rho)-\frac{e^{\varphi}}{\left(1+e^{\varphi}\right)^{2}}=\left(\frac{e^{\varphi}}{1+e^{\varphi}}-\rho\right)\left(\rho-\frac{1}{1+e^{\varphi}}\right)
$$

We then deduce

$$
\widehat{\mathbb{H}}(\rho, \varphi)=\left\langle\frac{e^{\varphi}}{1+e^{\varphi}}-\rho, \varepsilon \varphi_{x x}+\varphi_{x}\left(1-\varepsilon \varphi_{x}\right)\left(\rho-\frac{1}{1+e^{\varphi}}\right)\right\rangle=0
$$

since $\varphi$ satisfies (2.11) a.e.

It remains to prove the super-solution statement, that is $\widehat{\mathbb{H}}(\rho, \varphi) \geq 0$ for any $\rho \in \widehat{M}$ and $\varphi \in D_{\mathrm{F}}^{-} \widehat{\Lambda}(\rho)$. To this end, consider first the case $\varphi \in \partial_{\mathrm{app}} \widehat{\Lambda}(\rho)$. By definition, there exists a sequence $\left\{\rho^{n}\right\} \subset L^{1}([0,1])$ converging to $\rho \in \widehat{M}$ strongly in $L^{1}([0,1])$ such that $\partial \widehat{\Lambda}\left(\rho^{n}\right)=\left\{\varphi^{n}\right\}$ and $\varphi^{n} \rightarrow \varphi$ weakly* in $L^{\infty}([0,1])$. In particular, $\varphi^{n} \in \widehat{\mathcal{P}}\left(\rho^{n}\right)$ and therefore, by the bounds stated at the beginning of this 
proof, $\varphi \in \widehat{\mathcal{P}}(\rho)$. By the computation presented above, we then deduce $\widehat{\mathbb{H}}(\rho, \varphi)=0$. We now consider the general case $\varphi \in D_{\mathrm{F}}^{-} \widehat{\Lambda}(\rho)=\partial \widehat{\Lambda}(\rho)$. The concavity of $\widehat{\mathbb{H}}(\rho, \cdot)$ implies $\widehat{\mathbb{H}}(\rho, \varphi) \geq 0$ for any $\varphi \in \operatorname{co} \partial_{\text {app }} \widehat{\Lambda}(\rho)$. In view of Theorem 4.8, it is now enough to take the weak* closure in $L^{\infty}([0,1])$. This is easily accomplished noticing there exist $\delta \in(0,1)$ and $C<\infty$ depending only on $\rho$ such that any $\varphi \in \operatorname{co} \partial_{\text {app }} \widehat{\Lambda}(\rho)$ satisfies $\delta \leq \varepsilon \varphi_{x} \leq 1-\delta$ and $\left|\varphi_{x x}\right|_{L^{\infty}} \leq C$.

Acknowledgements. L. Bertini acknowledges the very kind hospitality at IMPA. D. Gabrielli acknowledges the financial support of PRIN 20078XYHVYS.

\section{REFERENCES}

[1] Arnold V.I.; Mathematical methods of classical mechanics, Second Edition. Springer-Verlag, New York 1989.

[2] Bardos C., le Roux A.Y., Nédélec J.-C.; First order quasilinear equations with boundary conditions. Comm. Partial Differential Equations 4, 1017-1034 (1979).

[3] Bellettini G., Bertini L., Mariani M., Novaga M.; Gamma-entropy cost for scalar conservation laws. Arch. Rational Mech. Anal. 195, 261-309 (2009).

[4] Bellettini G., Caselli F., Mariani M.; Quasi-potentials of the entropy functionals for scalar conservation laws. J. Funct. Analysis 258, 534-558 (2010).

[5] Bertini L., De Sole A., Gabrielli D., Jona-Lasinio G., Landim C.; Macroscopic fluctuation theory for stationary non equilibrium state. J. Statist. Phys. 110, 635-675 (2002).

[6] Bertini L., De Sole A., Gabrielli D., Jona-Lasinio G., Landim C.; Large deviations for the boundary driven symmetric simple exclusion process. Math. Phys. Anal. Geom. 6, 231-267 (2003).

[7] Bertini L., De Sole A., Gabrielli D., Jona-Lasinio G., Landim C.; Stochastic interacting particle systems out of equilibrium. J. Stat. Mech. Theory Exp. 7, P07014 (2007).

[8] Bertini L., Gabrielli D., Landim C.; Strong asymmetric limit of the quasi-potential of the boundary driven weakly asymmetric exclusion process. Commun. Math. Phys. 289, 311-334 (2009).

[9] Bertini L., Gabrielli D., Lebowitz J.L.; Large deviations for a stochastic model of heat flow. J. Statist. Phys. 121, 843-885 (2005).

[10] Bertini L., Landim C., Mourragui M.; Dynamical large deviations for the boundary driven weakly asymmetric exclusion process. Ann. Probab. 37, 2357-2403 (2009).

[11] Bodineau T., Giacomin G.; From dynamic to static large deviations in boundary driven exclusion particles systems. Stoch. Proc. Appl. 110, 67-81 (2004).

[12] Braides A.; Г-convergence for beginners. Oxford University Press, Oxford 2002.

[13] Day M.V.; On Lagrange manifolds and viscosity solutions. J. Math. Systems Estim. Control 8, 1-37 (1998).

[14] Day M.V., Darden T.A.; Some regularity results on the Ventcel-Freidlin quasi-potential function. Appl. Math. Optim. 13, 259-282 (1985).

[15] De Groen P.P.H., Karadzhov G.E.; Exponentially slow travelling waves on a finite interval for Burgers' type equation. Electronic J. Diff. Equations 1998, 1-38 (1998).

[16] Derrida B.; Non equilibrium steady states: fluctuations and large deviations of the density and of the current. J. Stat. Mech. Theory Exp. 7, P07023 (2007).

[17] Derrida B., Lebowitz J.L., Speer E.R.; Exact large deviation functional of a stationary open driven diffusive system: the asymmetric exclusion process. J. Statist. Phys. 110, 775-810 (2003).

[18] Dorfman J.R., Kirkpatrick T.R., Sengers J.V.; Generic long-range correlations in molecular fluids. Annu. Rev. Phys. Chem. 45, 213-239 (1994).

[19] Ekeland I., Temam R.; Convex analysis and variational problems. North-Holland, Amsterdam 1976.

[20] Enaud C., Derrida B.; Large deviation functional of the weakly asymmetric exclusion process. J. Statist. Phys. 114, 537-562 (2004). 
[21] Farfan J.; Static large deviations of boundary driven exclusion processes. Preprint 2009, arXiv:0908.1798v1

[22] Farfan J., Landim C., Mourragui M.; Hydrostatics and dynamical large deviations of boundary driven gradient symmetric exclusion processes. Preprint 2009, arXiv:0903.5526v1

[23] Freidlin M.I., Wentzell A.D.; Random perturbations of dynamical systems. Springer-Verlag, New York 1984.

[24] Graham R., Tél T,; Weak-noise limit of Fokker-Planck models and nondifferentiable potentials for dissipative dynamical systems. Phys. Rev. A 31, 1109-1122 (1985).

[25] Jauslin H.R.; Nondifferentiable potentials for nonequilibrium steady states. Phys. A 144, 179-191 (1987).

[26] Kipnis C., Landim C.; Scaling limits of interacting particle systems. Springer-Verlag, Berlin, 1999.

[27] Kipnis C., Olla S., Varadhan S.R.S., Hydrodynamics and large deviations for simple exclusion processes. Commun. Pure Appl. Math. 42, 115-137 (1989).

[28] Ladyženskaja O.A., Solonnikov V.A., Ural'ceva N.N.; Linear and quasilinear equations of parabolic type. Translations of Mathematical Monographs, Vol. 23 American Mathematical Society, Providence, R.I. 1967.

[29] Lanford O.E. III., Robinson D.W.; Statistical mechanics of quantum spin systems. III. Comm. Math. Phys. 9, 327-338 (1968).

[30] Mariani M.; Large Deviations Principles for Stochastic Scalar Conservation Laws. Probab. Theory Relat. Fields, to appear (2010), arXiv:0804.0997

[31] Meier R.S., Stein D.L.; A scaling theory of bifurcations in the symmetric weak noise escape problem. J. Statist. Phys. 83, 291-357 (1996).

[32] Perthame B.; Perturbed dynamical systems with an attracting singularity and weak viscosity limits in Hamilton-Jacobi equations. Trans. Amer. Math. Soc. 317, 723-748 (1990).

[33] Protter M.H., Weinberger H.F.; Maximum principles in differential equations. Corrected reprint of the 1967 original. Springer-Verlag, New York, 1984.

[34] Serre D.; Systems of conservation laws. 1 and 2. Cambridge University Press, Cambridge, 1999.

LORENZO BERTINI

Dipartimento di Matematica, Università di Roma 'La Sapienza'

P.le Aldo Moro 2, 00185 Roma, Italy

E-mail address: bertini@mat.uniroma1.it

Alberto De Sole

Dipartimento di Matematica, Università di Roma 'La Sapienza'

P.Le Aldo Moro 2, 00185 Roma, Italy

E-mail address: desole@mat.uniroma1.it

DAVIDE Gabrielli

Dipartimento di Matematica, Università dell'Aquila

67100 Coppito, L'Aquila, Italy

E-mail address: gabriell@univaq.it

Giovanni Jona-Lasinio

Dipartimento di Fisica and INFN, Università di Roma La Sapienza

P.le A. Moro 2, 00185 Roma, Italy

E-mail address: gianni.jona@roma1.infn.it

Claudio Landim

IMPA

Estrada Dona Castorina 110,

J. Botanico, 22460 Rio de Janeiro, Brazil

and

CNRS UMR 6085, Université DE Rouen,

Avenue de l'Université, BP.12, Technopôle du Madrillet, F76801 Saint-Étienne-Du-Rouvray, France.

E-mail address: landim@impa.br 